BNL- 52438

UC-414

AGS/AD/94-6

FORMAL

\title{
RF BEAM CONTROL FOR THE AGS BOOSTER
}

\author{
J. Michael Brennan
}

September 26, 1994

\section{ALTERNATING GRADIENT SYNCHROTRON DEPARTMENT \\ BROOKHAVEN NATIONAL LABORATORY ASSOCIATED UNIVERSITIES, INC. UPTON, LONG ISLAND, NEW YORK}

UNDER CONTRACT NO. DE-AC02-76CH00016 WITH THE UNITED STATES DEPARTMENT OF ENERGY

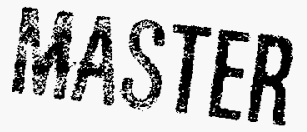




\section{DISCLAIMER}

This report was prepared as an account of work sponsored by the United States Government. Neither the United States nor the United States Department of Energy, nor any of their employees, nor any of their contractors, subcontractors, or their employees, makes any warranty, express or implied, or assumes any legal liability or responsibility for the accuracy, completeness, or usefulness of any information, apparatus, product or process disclosed, or represents that its use would not infringe privately owned rights. 


\section{DISCLAIMER}

This report was prepared as an account of work sponsored by an agency of the United States Government. Neither the United States Government nor any agency thereof, nor any of their employees, make any warranty, express or implied, or assumes any legal liability or responsibility for the accuracy, completeness, or usefulness of any information, apparatus, product, or process disclosed, or represents that its use would not infringe privately owned rights. Reference herein to any specific commercial product, process, or service by trade name, trademark, manufacturer, or otherwise does not necessarily constitute or imply its endorsement, recommendation, or favoring by the United States Government or any agency thereof. The views and opinions of authors expressed herein do not necessarily state or reflect those of the United States Government or any agency thereof. 


\section{DISCLAIMER}

Portions of this document may be illegible in electronic image products. Images are produced from the best available original document. 


\section{TABLE OF CONTENTS}

$\underline{\text { Page }}$

Introduction $\ldots \ldots \ldots \ldots \ldots \ldots \ldots \ldots \ldots \ldots \ldots \ldots \ldots \ldots \ldots \ldots$

1. General Description

1.1 Philosophy of the Design . . . . . . . . . . . . . 2

1.2 General Configuration of the System $\ldots \ldots \ldots \ldots \ldots \ldots$

1.2.1 Feedback Loops and System Variables . . . . . . . . . 3

1.2.2 Beam Transfer Functions . . . . . . . . . . . . 7

1.3 Beam Observation, the Pick-ups $\ldots \ldots \ldots \ldots \ldots \ldots \ldots \ldots 12$

1.4 The High Level System $\ldots \ldots \ldots \ldots \ldots \ldots \ldots$

1.4 .1 Cavities . . . . . . . . . . . . . . 14

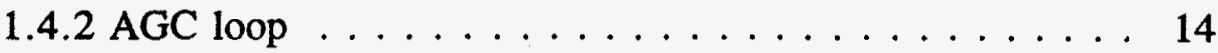

1.4.3 Phasing of the Cavities ............. 21



2. The Frequency Program

2.1 Revolution Frequency Table . . . . . . . . . . . . . 24

2.2 RF Frequency Generation . . . . . . . . . . . . 27

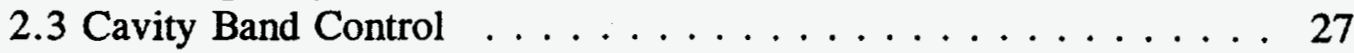

2.4 The Local Oscillator . . . . . . . . . . . . . . . 28

3. Heterodyne System

3.1 Motivation . . . . . . . . . . . . . . . . . 29

3.2 Hardware . . . . . . . . . . . . . . . . . . 30



3.2 .2 Input Mixer $\ldots \ldots \ldots \ldots \ldots \ldots \ldots \ldots \ldots \ldots$

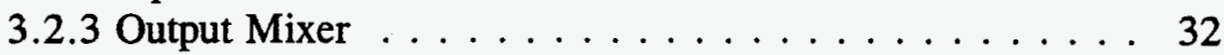

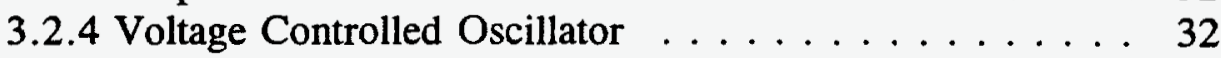

3.2.5 Phase Shifters . . . . . . . . . . . . . . . 32

3.2.6 Local Oscillator for Radius Measurement . . . . . . . . 37

4. Beam Control Feedback Loops

4.1 Phase Loop . . . . . . . . . . . . . . . . . . . 38

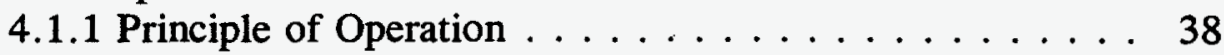

4.1.2 Design of the Phase Loop . . . . . . . . . . 39

4.1.3 Phase Loop Compensator . . . . . . . . . . . . . . . 45

4.2 Radial Loop . . . . . . . . . . . . . . . . . . . 60

4.2.1 Principle of Operation . . . . . . . . . . . . 60

4.2.2 Dynamics of the Radial Loop . . . . . . . . . . 61

4.2.3 Low Frequency Errors . . . . . . . . . . . . . 71

4.3 Frequency or Phase Control $\ldots \ldots \ldots \ldots \ldots \ldots \ldots$

4.3.1 Frequency Loop . . . . . . . . . . . . . . . . 74

4.3.2 Synchronization Loop . . . . . . . . . . . . . 79 
5. Synchronization and Cogging

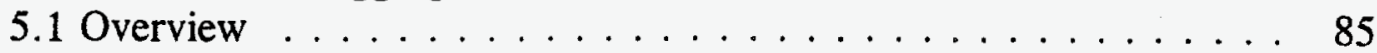

5.2 Cogging $\ldots \ldots \ldots \ldots \ldots \ldots \ldots \ldots \ldots \ldots \ldots$

5.3 Moving Reference Frame . . . . . . . . . . . . . . 89

5.3.1 Generation of the Moving Reference Frame ....... 91

5.3.2 Translation of Beam Frequency to Moving Reference Frame . . . . . . . . . . . . . . . 93

5.4 Frequency/Phase Detector . . . . . . . . . . . . . 95

5.5 Lock Acquisition in the Synchronization Loop . . . . . . . . . 96

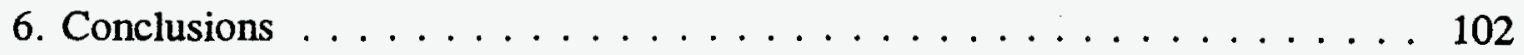

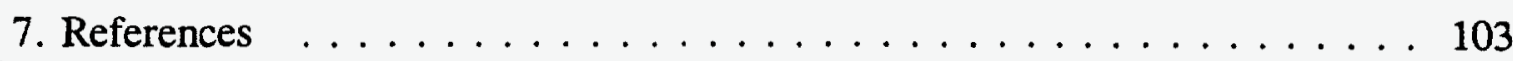

8. Appendix . . . . . . . . . . . . . . . . . . . . . . . 104

8.1 Final Value Theorem for the Radial Loop . . . . . . . . . . . 104

8.2 Feedback Loop Calculations with Program CC . . . . . . . . 106

8.2.1 Sensitivity Constants . . . . . . . . . . . . . 106

8.2.2 Program CC Transfer Function Names . . . . . . . . 107

8.2.3 Program CC Transfer Functions for Subsystems . . . . . 109

8.2.4 Calculation of Phase Response to Radial Step . . . . . . 111 


\section{INTRODUCTION}

$\mathrm{RF}$ beam control systems for hadron synchrotrons have evolved over the past three decades[1-6] into an essentially standard design. The key difference between hadron and lepton machines is the absence of radiation damping and existence of significant frequency variation in the case of hadrons. Although the motion of the hadron in the potential well of the rf wave is inherently stable it is not strongly damped. Damping must be provided by electronic feedback through the accelerating system. This feedback is typically called the phase loop. Frequency variation is usually accomplished by a combination of an open-loop approximation to the frequency, based on a measurement of the magnetic rigidity, and closed-loop feedback, based on a measurement of a beam parameter, such as, radius, frequency, or phase.

The technology of the if beam control system for the AGS Booster synchrotron is described. First, the overall philosophy of the design is explained in terms of a conventional servo system that regulates the beam horizontal position in the vacuum chamber. The concept of beam transfer functions is fundamental to the mathematics of the design process and is reviewed. The beam transfer functions required for this design are derived from first principles. An overview of the beam signal pick-ups and high level rf equipment is given.

The major subsystems, the frequency program, the heterodyne system, and beam feedback loops, are described in detail in Chapters 2, 3, and 4.

Beyond accelerating the beam, the rf system must also synchronize the bunches in the Booster to the buckets in the AGS before transfer. The technical challenge in this process is heightened by the need to accomplish synchronization while the frequency is still changing. Details of the synchronization system are given in Chapter 5.

This report is intended to serve two purposes. One is to document the hardware and performance of the systems that have been built. The other is to serve as a tutorial vehicle from which the non-expert can not only learn the details of this system but also learn the principles of beam control that have led to the particular design choices made. 


\section{GENERAL DESCRIPTION}

\subsection{Philosophy of the Design}

The Beam Control System is essentially a servo loop that keeps the beam at a prescribed radius. The reference input into the servo is a time-domain function specifying the desired radius. In servo language the loop suffers one main disturbance, that is, the magnetic field in the synchrotron changes. If the magnetic field increases the beam momentum must also increase if the radius is to remain at the specified value. Viewed in this light the entire acceleration system is simply a slave to the magnet. A priori, no assumptions are made about how the magnetic field will change with time. The central benefit of this philosophy of design is operational stability and convenience.

While on one hand the conceptual view of the system is that of a tracking servo, on the other hand most of the technical details of system are in place in order to compensate, in a open-loop or feedforward sense, any disturbance of the servo loop. For example, the accelerating frequency is approximated very accurately from a direct measurement of the magnetic field. Also, the drive power needed to attain a certain accelerating voltage is determined off-line and embodied in a non-linear function in the rf drive chain. The imperfections of these approximations are corrected by the servo system.

Unburdened of making first order corrections to the system the servo system is able to do a better job of its essential chores, that is, correcting uncontrollable inaccuracies in the feedforward systems (e.g., one does not measure the actual average magnetic field around the whole ring) and modifying the dynamics of beam response to the accelerating voltage.

The system operates in a Pulse by Pulse Modulation mode. Therefore, parameters that are required for making open-loop adjustments to the system (e.g. , the revolution frequency as a function of magnetic field) must be stored and retrievable within the $40 \mathrm{~ms}$ between the time that the next mode is specified and the time the rf system turns on for the new pulse. 


\subsection{General Configuration of the System}

1.2.1 Feedback Loops and System Variables. The general configuration of the system is described by figure 1.1. The elements of the diagram do not correspond directly to hardware components but show the sense of signal flow through the system. The elements of the diagram are: 1. the acceleration cavities (details related to the actual number of cavities, their relative phases, and regulation of the accelerating voltage are given below), 2 . beam transfer functions, relating responses of the beam phase, frequency, and radius to modulations of the rf frequency, 3. longitudinal beam pick-up, 4. transverse beam pick-up, 5. Voltage Controlled Oscillator, used to modulate the rf frequency of the cavities, 6 . phase detector, used to detect changes in the relative phase of the beam with respect to the accelerating voltage, 7 and 8 . feedback amplifiers for phase and radial feedback, 9. voltage controlled phase shifter driven by the radial feedback, 10. phase shifter, used to feedforward the synchronous phase angle from a measurement of the accelerating voltage and the rate of rise of the magnetic field, 11. delays in the signal path (about 4 microseconds).

The key dynamical variables of the system are indicated the figure. They are:

$\varphi_{b}$ - the phase of the beam bunch with respect to the net effective if voltage per turn. Careful matching of cable lengths from the beam pick-up (3) and the cavity voltage pick-up (1) is necessary in order for the observed phase to have absolute meaning as the rf frequency changes.

$\varphi_{\mathrm{s}}$ - the synchronous phase angle. This is calculated from on-line measurements to the $\mathrm{dB} / \mathrm{dt}$ and rf voltage.

$\varphi$ - the instantaneous phase deviation of the bunch from the synchronous phase. This is the variable of the linearized model of the coherent bunch motion developed below.

$\varphi_{\mathrm{r}}$ - the phase correction introduced by the radial feedback. The nominal value of this variable is zero. It will differ from zero momentarily while a radial change is being made. When the new radius is stable it returns to zero. Its small dc (low frequency) value compensates for errors in; the synchronous phase calculation, dc errors in the feedback amplifier and VCO, and miss-matches in cable lengths.

$\varphi_{\mathrm{e}}$ - the phase error. The feedback loop has very high gain at low frequency in order to keep this variable equal to zero. 


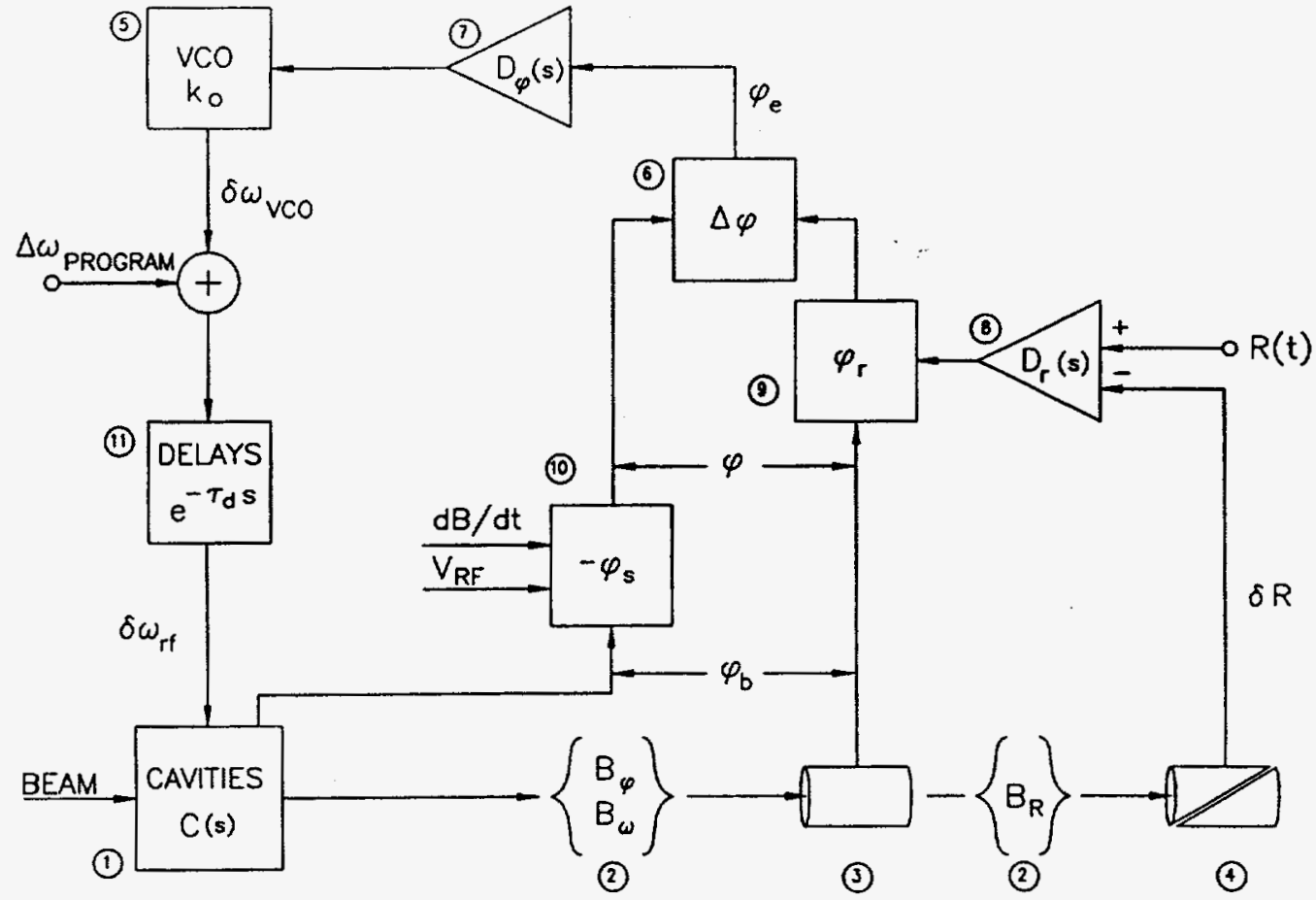

Figure 1.1. The concept of the beam control system. Because the two phase shifters, numbers 9 and 10 , are before the phase detector, number 6 , its nominal output is zero. See text for detailed description of the numbered components and labeled variables. 
$\Delta \omega_{\text {program }}$ - this represents the errors in the frequency program. Since the program changes in discrete steps, there will always be errors in the program at least as large as half the step size.

$\delta \omega_{\mathrm{Vco}}$ - modulations in the output frequency of the VCO, caused by the action of the feedback loops.

$\delta \omega_{\mathrm{rf}}$ - modulations in the frequency of the drive signal to the power amplifiers and cavities. The actual frequency felt by the beam is further modified by the cavity response function, $\mathrm{C}(\mathrm{s})$.

$\delta \mathrm{R}$ - variations of the beam radius from the center line of the transverse pickups.

Figure 1.1 is redrawn in Figure 1.2 as a classical servo system with $R(t)$, the reference value for the beam radius, as the main input. The output is the beam frequency, proportional to radius. One can see from this configuration that the phase correction, $\varphi_{r}$, serves as a reference to the embedded loop, the phase loop.

Consider the quiescent state, the low frequency gain of the phase loop amplifier is high, which implies the phase error $\varphi_{\mathrm{e}}$ is zero. If the beam is being accelerated $\varphi_{\mathrm{s}}$ is not zero but under the assumption that the calculation of $\varphi_{\mathrm{s}}$ and the $\varphi_{\mathrm{s}}$ phase shifter are accurate then the phase shifter $\varphi_{\mathrm{r}}$ is zero. The actual beam radius, $\delta \mathrm{R}$, equals the reference radius, $\mathrm{R}(\mathrm{t})$, and the phase correction, $\varphi_{\mathrm{I}}$ is zero.

The system is disturbed from equilibrium by a change at any of the inputs: the reference radius, $R(t)$, the frequency program, $\Delta \omega_{\text {program }}$, the accelerating voltage, $\mathrm{V}_{\mathrm{rf}}$, or the magnetic field rate of rise, $\mathrm{dB} / \mathrm{dt}$. In each case $\varphi_{\mathrm{e}}$ will deviate from zero causing the VCO to go to a new frequency. The loops close through the response of the beam to the change in accelerating frequency. Ultimately the beam phase, $\varphi_{b}$, will change in such a way to return $\varphi_{e}$ to zero. 




Figure 1.2. The beam control system seen as a classical servo system. The phase loop, with feedback via the beam transfer function $\mathrm{B} \varphi$, is nested within the radial loop which gets feedback via $B_{R}$. 
1.2.2 Beam Transfer Functions. The key to understanding why the feedback loops are configured in the manner shown is to examine the beam response functions. I make the approximation that the beam can be considered a single macro-particle and that the motion can be linearized by treating small oscillations about the stable synchronous phase angle. These approximation describe well the behavior of the beam when the loops are regulating. One defines three response functions which give the changes in beam parameters, phase, frequency, and radius as a result of a change in the frequency of the accelerating voltage.

$$
\begin{gathered}
\varphi_{b}=B_{\varphi} \delta \omega_{r f} \\
\delta \omega_{b}=B_{\omega} \delta \omega_{r f} \\
\delta R=B_{R} \delta \omega_{r f}
\end{gathered}
$$

The response functions can be derived from the basic first order equations of the synchrotron motion:

$$
\begin{gathered}
\frac{d}{d t}(\delta E)=\frac{1}{T_{r e v}} e V_{r f}\left[\sin \left(\varphi_{s}+\varphi\right)-\sin \varphi_{s}\right] \\
\frac{d}{d t}(\varphi)=\frac{1}{T_{r e v}}\left[\left(2 \pi \frac{\delta t}{T_{r f}}+\delta \omega_{r f} T_{r e v}\right]\right.
\end{gathered}
$$

where:

$\varphi_{\mathrm{S}}$ is the synchronous phase angle;

$V_{\mathrm{rf}}$ is the voltage of the accelerating cavities;

$\mathrm{T}_{\text {rev }}$ is the revolution period;

$\delta t$ is the deviation in revolution period of the macro-particle from the synchronous particle;

$\delta \omega_{\mathrm{rf}}$ is the deviation of the $\mathrm{rf}$ frequency from the bunch frequency of the synchronous particle;

and

$\delta \mathrm{E}$ is the energy deviation of the macro-particle from the synchronous energy (synchronous energy =energy of the particle that returns to same phase, $\varphi_{s}$, each revolution);

$\varphi$ is the deviation of the phase of the macro-particle from $\varphi_{\mathrm{s}}$. 
The first equation can be linearized by expanding the sine function. One gets,

$$
\frac{d}{d t}(\delta E)=\left[\frac{e V_{r f}}{T_{r e v}} \cos \varphi_{s}\right] \varphi
$$

The second equation contains $\delta \mathrm{E}$ through $\delta \mathrm{t}$. The relationship can be found from,

$$
\left(T_{r e v}+\delta t\right)=\frac{2 \pi(R+\delta R)}{(\beta+\delta \beta) c},
$$

and

$$
\frac{\delta \beta}{\beta}=\frac{1}{\gamma^{2} \beta^{2}} \frac{\delta E}{E} ; \quad \frac{\delta R}{R}=\frac{1}{\gamma_{t r}^{2} \beta^{2}} \frac{\delta E}{E} .
$$

One gets,

$$
\frac{d}{d t}(\varphi)=\frac{\omega_{r f} \eta}{\beta^{2} E} \delta E+\delta \omega_{r f},
$$

Where:

$$
\eta=\frac{1}{\gamma_{t r}^{2}}-\frac{1}{\gamma^{2}}
$$

The term with $\delta \omega_{\mathrm{rf}}$ is the key term here. It expresses the rate of phase slip between the if voltage and the beam revolution period. It is a driving term in this system of first order differential equations. To obtain the beam response functions take the Laplace transforms, assume zero initial conditions, and solve algebraically for $\varphi$ to get,

$$
\varphi(s)=\frac{s}{s^{2}+\omega_{s}^{2}} \delta \omega_{r f}(s),
$$


where:

$$
\begin{gathered}
\omega_{s}^{2}=\left(h e V_{r f}\right)\left[\frac{\omega_{r e v}}{\beta}\right]^{2}\left[\frac{|\eta| \cos \varphi_{s}}{2 \pi E}\right], \\
h=\text { harmonic number, } \omega_{r f} / \omega_{r e v} .
\end{gathered}
$$

The other response functions are obtained by solving for $\delta \mathrm{E}$, and recalling that frequency and radius deviations are proportional to $\delta \mathrm{E}$ through,

$$
\frac{\delta R}{R}=\frac{1}{\gamma_{t}^{2} \beta^{2}} \frac{\delta E}{E}
$$

and

$$
\frac{\delta \omega_{b}}{\omega_{b}}=-\frac{\eta}{\beta^{2}} \frac{\delta E}{E} .
$$

One gets,

$$
\begin{gathered}
\delta R(s)=\frac{b}{s^{2}+\omega_{s}^{2}} \delta \omega_{r f}(s) ; \quad b=\frac{c e V_{r f} \cos \varphi_{s}}{2 \pi \beta \gamma_{t r}^{2} E} \\
\delta \omega_{b}(s)=\frac{\omega_{s}^{2}}{s^{2}+\omega_{s}^{2}} \delta \omega_{r f}(s) .
\end{gathered}
$$


The three beam response functions then are:

$$
\begin{aligned}
& B_{\varphi}(s)=\frac{s}{s^{2}+\omega_{s}^{2}} ; \\
& B_{R}(s)=\frac{b}{s^{2}+\omega_{s}^{2}} ; \\
& B_{\omega}(s)=\frac{\omega_{s}^{2}}{s^{2}+\omega_{s}^{2}} .
\end{aligned}
$$

These are response functions of pure, undamped, sines and cosines (the poles are on the imaginary axis).

Feedback through radial response will not change this in a fundamental way. To see this, write the closed loop response for radial feedback only. Consider Figure 1.2 and neglect the inner loop via $\mathrm{B} \varphi$.

$$
\delta R=\frac{k B_{R}}{1+k B_{R}},
$$

where $\mathrm{k}$ represents the gain through the whole loop, except for the beam response.

Using Equation 1.4 gives,

$$
\delta R=\frac{k b}{s^{2}+\omega_{s}^{2}+k b} R .
$$

If one only had feedback on the radius the closed loop response would not be fundamentally different from the open loop response. The characteristic frequency of the system would be shifted up by $\omega_{s}^{2} \rightarrow \omega_{s}^{2}+k b$, but the motion would still be fundamentally undamped. 
The phase feedback loop, however, changes the basic character of the motion. The closed loop response of the phase loop is (the inner loop of Figure 1.2 , not the same $\mathrm{k}$ as above)

$$
\varphi=\frac{k B_{\varphi}}{1+k B_{\varphi}} \varphi_{r} .
$$

Using Equation 1.3 gives,

$$
\varphi=\frac{k s}{s^{2}+k s+\omega_{s}^{2}} \varphi_{r} .
$$

This corresponds to damped motion. The linear term in s gives damping, as in viscosity, friction, or resistance. The poles of this function are complex, with negative real parts (depending on the sign of $k$ ). The phase loop tames the beam response through this damping so that the radial servo can be a welldamped system.

The phase loop is the heart of the Beam Control system. As will be shown below the choice of radius as the main controlled parameter of the machine is somewhat arbitrary. In certain cases it is advantageous to regulate the frequency or the absolute phase of the beam, in favor of the radius. But in these cases also the phase loop still operates to modify the basic beam response to that of a welldamped second-order system.

The penalty for not damping the beam response with the phase loop goes beyond its effect on the other loops. They could, in principle, be stabilized with some sort of loop compensation. It is the beam itself that would be adversely affected by under-damped, oscillatory motion in the longitudinal phase space of the synchrotron. The approximation of small oscillations would break down and non-linear effects from the rf waveform would drive filimentation, emittance growth, and ultimately beam loss. 


\subsection{Beam Observation, the Pick-Ups}

The key to successful beam control is quality beam observation. There are two observation systems. One picks up the transverse position of the beam in the vacuum chamber, and the other picks up its longitudinal position with respect to the rf accelerating wave. The choice of the technology for these pick-up systems was driven by the requirements that the outputs should be correct over a wide range in beam intensity, $\mathrm{rf}$ frequency, and bunch shape.

The transverse position (radius) is given by processing circuits employing the AM-to-PM technique.[7] These circuits are fed by signals from front-end amplifiers of the Beam Position Monitoring system, which are connected to pairs of split-cylinder pick-up devices. [8] The AM-to-PM technique is intrinsically selfnormalizing with respect to beam intensity, giving an output that is proportional to the ratio of the two inputs. Bench measurements have shown the ratio to be independent of the average signal level over more than three decades. Frequency independence is achieved by shifting the frequency of the beam signal to a fixed intermediate frequency of $10.7 \mathrm{MHz}$ before processing. Sensitivity is enhanced by employing narrowband filters at the intermediate frequency to improve the signal-to-noise ratio. The processing circuits are located in the ring, and are electrically connected to the beam pipe at the pick-ups. The output, processed, position signals are sent to the rf control electronics via fiber optic cables, operating in a voltage-to-frequency/frequency-to-voltage encoding scheme. The carrier frequency is $9 \pm 1 \mathrm{MHz}$, and the system bandwidth is $50 \mathrm{kHz}$.

Two pick-ups are used and their average value is taken as the measure of the radius of the beam orbit. The locations for the two pick-ups are chosen to be approximately one-half a betatron wave length apart at the nominal tune of the machine. In this way one gets a first order cancellation of orbit distortions, which otherwise would be interpreted as changes in the average orbit. The cancellation of the orbit distortions relies on the assumption that the point of orbit perturbation does not lie between the two pick-ups. In the Booster the locations A2 and A8 were chosen because there are no intentional orbit bumps between them. In addition the lattice beta and dispersion functions are approximately equal at these locations.

The longitudinal position signal is taken from a resistive wall gap type detector (Wall Current Monitor).[9, 10] This is a passive device and thereby capable of a very wide dynamic range in beam intensity. It is low impedance device ( $31 / 8 \mathrm{Ohms}$ ) and therefore inherently low noise. The bandwidth of the pick-up is also very wide, $20 \mathrm{kHz}$ to more than $1 \mathrm{GHz}$. An important feature of this type of pick-up is its tolerance to beam lost in the vacuum chamber. This comes from its large physical size and low impedance $(31 / 8 \mathrm{Ohm}$ within its bandwidth and zero Ohms at dc). The output signal from the Wall Current Monitor is sent to the $\mathrm{rf}$ control electronics via a low-loss ( $7 / 8$ inch diameter) 
semi-rigid coaxial cable. The pick-up is a current measuring device so even for a fixed amount of beam charge in the machine the magnitude of the signal may change over a wide range during the acceleration cycle as the beam speed increase implies a current increase. 


\section{$1.4 \quad$ The High Level System}

1.4.1 Cavities. Four ferrite-tuned rf cavities are available in the Booster. Two of the cavities operate over the frequency range 0.6 to $3.0 \mathrm{MHz}$, supplying $17 \mathrm{kV}$ each and the other two cavities operate from 2.3 to $5.0 \mathrm{MHz}$, supplying $45 \mathrm{kV}$ each. The wide frequency range is provided to accommodate heavy ions preaccelerated in the Tandem Van de Graaff, giving ion speeds as low as $0.04 \mathrm{c}$. The higher frequency cavities are used to accelerate protons from the $200 \mathrm{MeV} \mathrm{H}^{-}$ linac, through the frequency range 2.5 to $4.1 \mathrm{MHz}$. The $90 \mathrm{kV}$ of total voltage in this frequency range provides a longitudinal acceptance at injection of $1.5 \mathrm{eV} \cdot \mathrm{s}$ in each of three bunches.

1.4.2 AGC Loop. The power amplifiers for the cavities operate in class $\mathrm{AB}$ and are drive modulated. They are capable of over $30 \mathrm{~dB}$ of dynamic range. The overall gain of the power chain is not linear however, due for the most part to rf level-dependent loss factors in the tuning ferrites. The ferrite losses also depend strongly on the rf frequency, and in the case of the higher frequency cavities, on the time rate of change of frequency, df/dt. In order to attain stability and precision of the cavities' voltage a feedback system is required. This system is called the AGC circuit (Automatic Gain Control).

A basic feature of the AGC system is careful limiting of its compliance while maintaining high feedback gain for precision. Compliance refers to the range through which the feedback is allowed to modify the drive power to the cavities in order to maintain the desired voltage. A typical failure mode of high power AGC systems is an over-power situation (often to the damage level) when the requested cavity voltage cannot be attained, or worse, the measurement of the voltage fails and the AGC system runs up the drive power to its maximum compliance.

In this AGC system the compliance is controlled in the following way. An operating point adjustment sets the factor by which the AGC system is allowed to increase the drive power, typically a factor of two $(+3 \mathrm{~dB})$. The same factor applies no matter what is the absolute level. In this way it is impossible to get a high drive power when a low value of cavity voltage is requested, irrespective of whether the AGC feedback is satisfied or not. On the power decreasing side, the range is not restricted, it is always at least $-60 \mathrm{~dB}$.

Since the range of power excursions allowed to the AGC system is limited, a programmed feedforward circuit automatically sets the nominal value of the drive power from a measurement of the requested cavity voltage and frequency. A "personality" curve has been measured for each cavity and is stored in a look-up PROM. The PROM is addressed by a digitized value of the requested cavity voltage. The data in the PROM scale the drive power. The PROM holds families of gain curves for different rf frequencies. The data in the PROM is addressed in a two-dimensional fashion, with one axis being voltage and the other axis being frequency. 


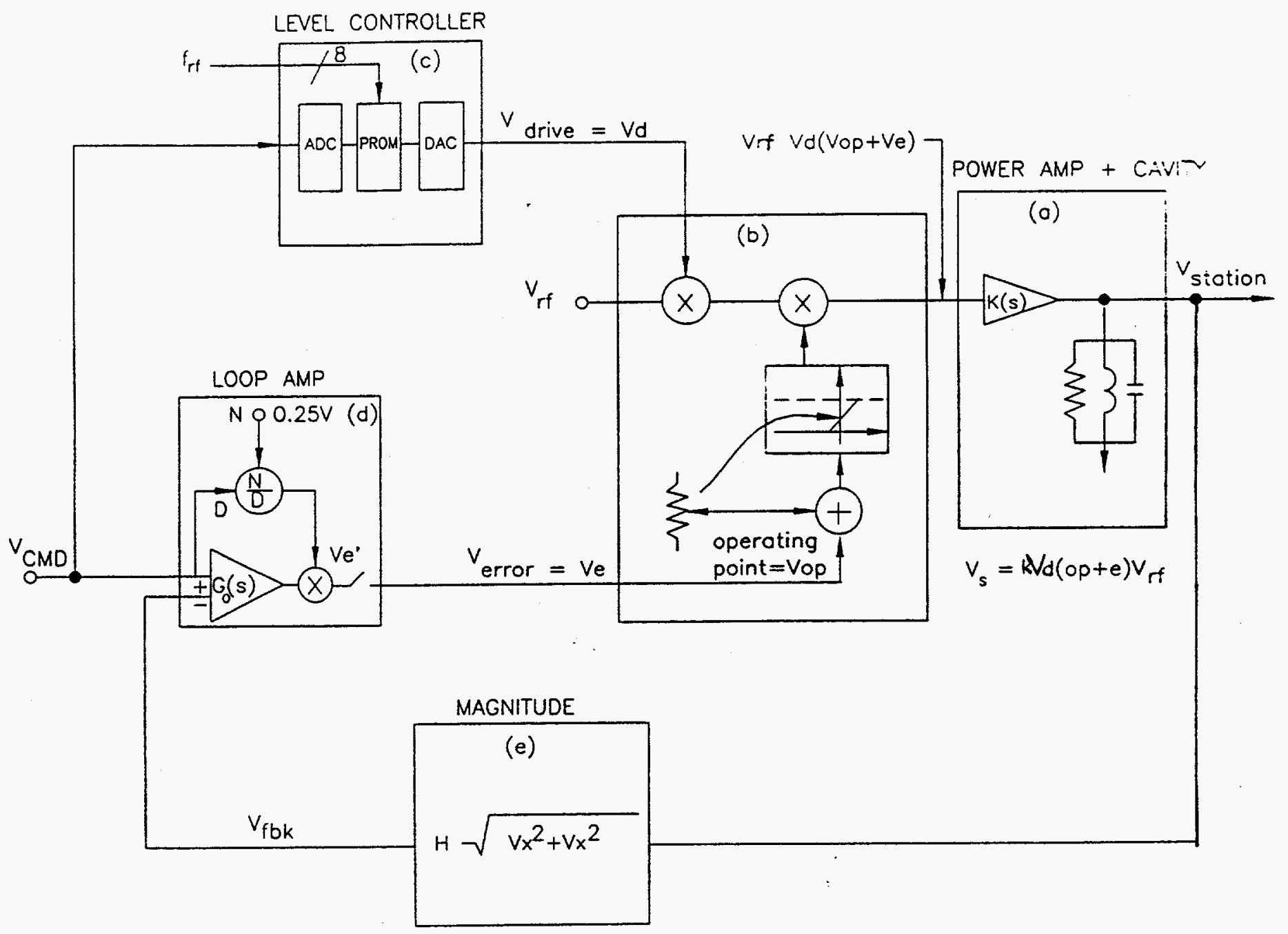

Figure 1.3. Block diagram of the Automatic Gain Control (AGC) system. The system has limited compliance via block $b$, and feedback loop gain independent of if level via block $d$. The magnitude circuit, block e, calculates the if magnitude directly as shown and does not use rectifier diodes and a low pass filter which would limit its bandwidth. 
Figure 1.3 shows the components of the AGC system; a. the power amplifier and cavity ( $\mathrm{V}_{\text {Station }}$ is the total voltage of the cavity, as distinguished from the voltage per gap or net voltage per turn around the ring), $b$. a section of the output mixer (see Section 3 below, Heterodyne System), c. the cavity level controller, with PROM, d. the loop amplifier, e. and the rf magnitude calculating circuit. The symbols indicating addition, multiplication and division are to be taken literally.

The output level of the output mixer is set by two linear modulators in cascade. The first sets the nominal drive level from data in the PROM. The second modulates the drive level to implement the AGC function. Indicated in the figure is the limiter that sets the operating point and determines the output level when the AGC error is zero, and also the range through which the drive may be increased. The figure shows that when the AGC error is negative the drive power can be decreased to zero. Operationally one chooses an operating point with the feedback loop open and then adjusts the rf gain of the power chain to get agreement between the actual cavity voltage and the requested voltage, $V_{C M D}$.

The effect of the linear modulators in cascade is to make the loop gain of servo system proportional to the rf voltage level in the cavity. Since the maximum gain is limited (by time delays and cavity response time) the loop's performance would be poor at low cavity voltage. To see why the loop gain would scale with rf level one calculates the loop gain by considering the result of a modulation injected into the loop at the switch, in Figure 1.3(d).

Let $V_{e}$ be the injected signal and $V_{e}^{\prime}$ be the resulting output of the loop amplifier. ( $G$ is the gain of the loop amplifier). Bold symbols represent the magnitude of rf signals.

$$
\begin{aligned}
& V_{e}^{\prime}=G\left(V_{C M D}-V_{f f k}\right)=G\left(V_{C M D}-H V_{s}\right) \\
& V_{s}=k\left(V_{o p}+V_{e}\right) V_{d} V_{r f} \\
& \Rightarrow V_{e}^{\prime}=G V_{C M D}-G H k\left(V_{o p}+V_{e}\right) V_{d} V_{r f} \\
& \text { Loop Gain } \quad=\partial V_{e}^{\prime} / \partial V_{e}=-G H k V_{d} V_{r f} \\
& \text { when } V_{e}=0 \Rightarrow V_{s}=k V_{o p} V_{d} V_{r f} \\
& \text { that is } k=V_{s} / V_{o p} V_{d} V_{r f}
\end{aligned}
$$

Therefore, Loop Gain $=-\mathrm{GH} \mathrm{V}_{\mathrm{s}} / \mathrm{V}_{\mathrm{op}}$. 
To offset this effect, the loop amplifier contains a scaling circuit that adjusts its gain according to

$$
G(s)=\frac{G_{o}(s)}{V_{C M D}} .
$$

Since $V_{C M D}$ is nominally equal to $H V_{s}$ the overall loop gain is therefore independent of cavity voltage level. Using $V_{C M D}$ is preferable to $V_{s}$ because $V_{s}$ is affected by the loop amplifier gain and so an unintended feedback loop would be created if $\mathbf{V}_{\mathbf{s}}$ were used.

From the diagram of Figure 1.3 one sees that the scaling is done in a two step process. First, 0.25 Volts $/ \mathrm{V}_{\mathrm{CMD}}$ is calculated with an analog divider. The numerator is set, via a stable reference, to 0.25 volts. The denominator is taken from the reference input and limited via a precision clamp circuit to $0.25<$ $\mathrm{V}_{\text {denominator }}<5.0$. This gives a 20 to 1 dynamic range over which the AGC loop gain is constant. The maximum cavity voltage of $50 \mathrm{kV} /$ Station limits the $\mathrm{V}_{\mathrm{CMD}}$ to less than 5 volts. Below $\mathrm{V}_{\mathrm{CMD}}=0.25$ Volts $\left(\mathrm{V}_{\text {Station }}=2.5 \mathrm{kV} /\right.$ Station $)$ the loop gain falls, but the loop stability is not compromised by the reduced gain.

After the divider, a linear multiplier scales the gain by $\left(0.25 / \mathrm{V}_{\mathrm{CMD}}\right)$. The process is done in two steps (first divide and then multiply) because the bandwidth of a multiplier is much greater than a divider (a divider has a feedback loop within it). In this way the divider is not in the path of the AGC feedback loop and only has to handle the relatively slow variations of $\mathrm{V}_{\mathrm{CMD}}$. The multiplier bandwidth exceeds $300 \mathrm{kHz}$.

The cavity voltage, $\mathbf{V}_{s}$, is measured with capacitive dividers, attached directly to one of the acceleration gaps. Scale factors are set to $5 \mathrm{kV} / \mathrm{gap} /$ volt for Band III cavities and $10 \mathrm{kV} / \mathrm{gap} / \mathrm{volt}$ for Band II. Since there two gaps per station for Band III and one gap per station for Band II this gives $10 \mathrm{kV} / \mathrm{station} /$ volt in both cases so that the signals can be combined directly to get the total voltage per turn.

The magnitude circuit does not use a diode detector. Instead it uses fast, four-quadrant, linear multipliers to compute $V_{x}{ }^{2}$ and $V_{y}{ }^{2}$, where $V_{x}$ and $V_{y}$ are obtained from a quadrature hybrid transformer. Adding $V_{x}{ }^{2}$ and $V_{y}{ }^{2}$ and calculating the square root via a high performance square rooter (Analog Devices $\mathrm{AD} 538$ ) gives an output linearly proportional to the cavity voltage level. The calibration of the circuit is adjusted to $10 \mathrm{kV} / \mathrm{Station}$ per volt out. The advantages of this technique are linearity over a wide dynamic range and high detection bandwidth, irrespective of $\mathrm{rf}$ frequency (no filtering of a rectified sinewave is required). 
The dynamics of the AGC loop are controlled by the loop amplifier, $G(s)$ in Figure 1.3. Two effects have to be accommodated: one is the delay between loop amplifier output and magnitude circuit output, and the other is the frequency response of the cavity rf resonance to amplitude modulations of the drive signal.

There are two sources of delay. Cable delays amount to approximately 1.4 microseconds. The magnitude circuit has an inherent delay of approximately 1.5 microseconds due to the square rooter.

The cavity amplitude modulation frequency response was measured with the Dynamic Signal Analyzer HP3562A at various if levels and frequencies. Figure 1.4 is an example at $30 \mathrm{kV} / \mathrm{station}$ in a Band III cavity. The response was found to be well characterized by a single pole roll-off with a corner frequency in the neighborhood of $30 \mathrm{kHz}$, and depending relatively weakly on rf frequency and voltage. The near independence of $\mathrm{rf}$ frequency may seem surprising at first thought since the time constant of the rf resonance decreases with frequency according to, $\tau=(2 \mathrm{Q} / \omega)$. However, in the first approximation (constant loss factor of the ferrites) $\mathrm{Q}$ increases with frequency, $\mathrm{Q}=\omega \mathrm{RC}$, canceling the frequency dependence.

Performance specifications for the AGC system are given by the following considerations. (1) Good dc accuracy for control of the cavity voltage. This would be very important, for example, in a cavity counter-phasing operation. A value of $40 \mathrm{~dB}$ (1\% error) at dc was taken. (2) Significant loop gain at twice the synchrotron frequency. This is important for the stability of high intensity beams. Bunch quadrupole mode oscillations can be driven by beam loading perturbations of the cavity voltages at twice the synchrotron frequency. The AGC system should suppress these perturbations by at least a factor of 10 . This implies a loop gain of $>10 \mathrm{~dB}$ at $14 \mathrm{kHz}$. (3) Overshoot and ringing on the step response of cavity voltage should be minimized since fast variation of the cavity voltage can be non-adiabatic and lead to emittance growth. This suggests a conservative phase margin of 45 degrees or greater.

Meeting these specifications requires a loop compensator with a low frequency pole and a high frequency zero. The AGC compensator was implemented with the conventional PID (Proportional plus Integral plus Derivative) characteristic. Roughly speaking the derivative term gives a zero to offset the cavity pole and delay. The integral term provides the high gain at dc, and the proportional term is used to set the gain around twice the synchrotron frequency.

Figure 1.5 is Bode plot of a measurement of the open loop gain for the system with a PID compensator given by,

$$
\begin{aligned}
G(s) & =k_{p}\left(1+\frac{1}{\tau_{I} s}+\tau_{D}\right) \\
\text { where } \mathrm{k}_{\mathrm{p}} & =40, \tau_{\mathrm{I}}=8 \times 10^{-6} \mathrm{~s}, \tau_{\mathrm{D}}=2 \times 10^{-6} \mathrm{~s} .
\end{aligned}
$$






Figure 1.4. The amplitude modulation frequency response of a cavity at $30 \mathrm{kV}$ and $2 \mathrm{MHz}$. The response is well described by a single pole low pass filter at $30 \mathrm{kHz}$. 


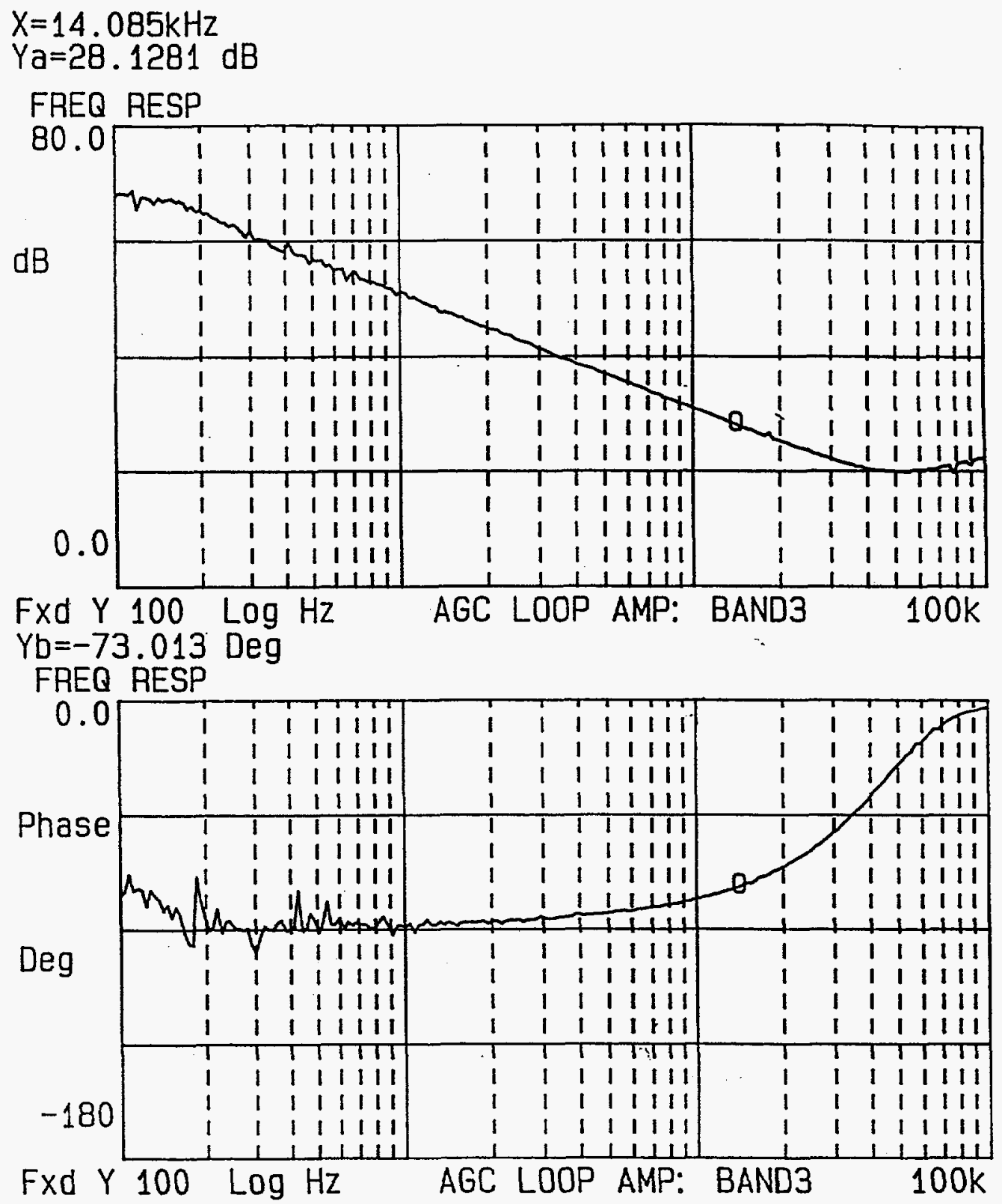

Figure 1.5. A measurement of the PID loop amplifier of the AGC system. PID constants are given in the text. 
1.4.3 Phasing of the Cavities. Table 1.1 gives the angular locations $(\theta)$ of the four cavities on the ring with respect to the longitudinal pick-up (wall current monitor at D6). The rf phase difference, modulo $2 \pi$, at various harmonic numbers is also shown.

TABLE 1.1

\begin{tabular}{||c|c|c|c|l|l|l||}
\hline \hline Cavity & $\begin{array}{c}\text { Frequency } \\
(\mathrm{MHz})\end{array}$ & $\begin{array}{c}\text { Angle }\left(^{\circ}\right) \\
(\theta)\end{array}$ & \multicolumn{4}{|c|}{ Phase $\left(^{\circ}\right)$} \\
\hline \multicolumn{1}{||c|}{} & $\mathrm{h}=1$ & $\mathrm{~h}=3$ & $\mathrm{~h}=6$ & $\mathrm{~h}=24$ & $\mathrm{~h}=48$ \\
\hline E6 & $2.3-5.0$ & 58.02 & 174.1 & 348.1 & 312.5 & 265.0 \\
\hline A3 & $0.6-3 . \mathrm{P}$ & 155.15 & 105.4 & 210.9 & 123.6 & 247.2 \\
\hline A6 & $2.3-5.0$ & 177.39 & 172.2 & 344.3 & 297.4 & 234.7 \\
\hline B3 & $0.6-3.0$ & 214.72 & 284.2 & 208.3 & 113.3 & 226.6 \\
\hline
\end{tabular}

From Table 1.1 one can see that the relative if phase of each cavity with respect to the pick-up is unique and furthermore that it changes significantly with rf harmonic number. To accommodate this effect each cavity drive circuit contains a fixed, frequency-independent phase shifter to set the drive phase for $\mathrm{h}=3$, and a digitally programmed phase shifter whose phase changes with harmonic number, according to $\varphi_{\mathrm{r}}=\mathrm{h} \theta$. 
1.4.4 Cavity Tuning Servos. The cavities are tuned to resonance by biasing the ferrites. Cavities A6 and E6 require $1200 \mathrm{~A}$ for the maximum frequency, and cavities A3 and A8 require 200 A. Each cavity has its own dc power supply and series-pass transistor bank for regulating the tuning current. Feedback regulates the tuning current as the rf frequency changes.

Figure 1.6 is a block diagram of the tuning system. The feedback consists of three nested loops. The first loop, labeled LEM1 (LEM is the trade name of the dc-coupled current transformer used to sense the current), regulates the current into the driver stage of the transistor bank. The second loop, labeled LEM2, regulates the current out of the bank.

The third loop regulates the if phase of the voltage at the anode of the final power tube compared to the voltage at the grid of the tube. The voltage at the grid is in phase with the current at the anode so that requiring these two voltages to be in phase is equivalent to requiring that the current and voltage at the anode are in phase. This is equivalent to requiring that the load of the tube is real. Without beam loading this insures that the cavity is on resonance. Under beam loaded conditions this will miss-tune the cavity such that the reactive component of the beam current will cancel the imbalance of circulating current between the inductive and capacitive branches of the cavity resonance. The power amplifier will be able to deliver its maximum power when its load appears real.

The benefit of the two inner loops is increased bandwidth and smaller residual tuning error. Feedback on the current has the effect of making the transistor bank a better current source, that is, a higher output resistance. Since the load is essentially inductive the higher output resistance leads to a lower $L / R$ time constant and greater bandwidth. The load is, in fact, not pure inductive. RF bypass capacitors in the cavity circuit appear in parallel with the inductance and cause a resonance at about $20 \mathrm{kHz}$. This resonance in the load circuit complicates the servo design.

The block diagram shows two "shapers". These are feedforward inputs to the system that approximate the tuning current from a measurement of the $\mathrm{rf}$ frequency, $\mathrm{f}-\mathrm{to}-\mathrm{V}$, and frequency change rate, $\mathrm{df} / \mathrm{dt}$. The cavity shaper realizes a parameterization of the cavity tuning curve, and so it is a reference to the LEM2 loop, which controls current out of the transistor bank. The bank shaper accommodates the non-linear characteristic of the current out of the transistor bank as a function of the driver current into the bank. It therefore is a reference to the LEM1 loop. The shapers minimize the error signals within the loop due to non-linearities and therefore provide more compliance in the error signals for beam related corrections.

A "ramp-down" circuit is shown, triggered by three possible events; end of the $\mathrm{rf}$ cycle, a system fault indication, and preparation for a switch of harmonic number. When triggered, the ramp-down circuit holds the level of the command to the current source and sums with it a decreasing linear ramp to zero. The rate of the ramp is set to match the maximum allowable $\mathrm{L} \mathrm{dI/dt}$ voltage for the transistor bank. 


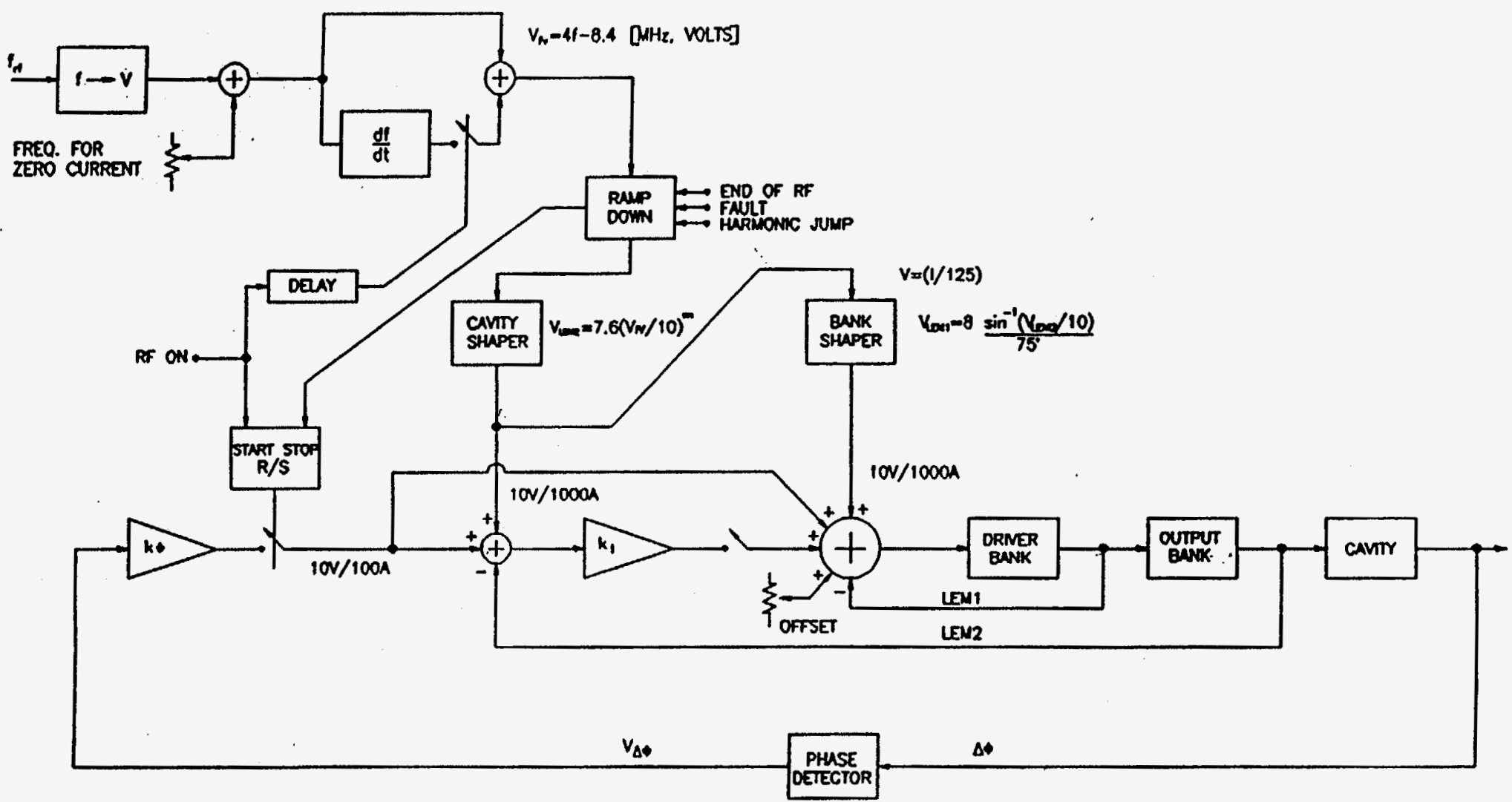

Figure 1.6. Block diagram of the cavity tuning system. There are three nested feedback loops, bank driver current, bank output current, and cavity rf impedance angle. Two shaper circuits, bank shaper and cavity shaper, supply feedforward programs within the loops. Maximum output current is $1500 \mathrm{~A}$. 


\section{THE FREQUENCY PROGRAM}

\subsection{Revolution Frequency Table}

The frequency program is the best estimate of rf frequency as function of the dipole magnetic field in the ring. The magnetic field is measured continuously throughout the acceleration cycle to the precision of 0.1 Gauss with the "Gauss Clock".[11] The measurement is made with a long search coil inside the vacuum chamber of a special, reference magnetic, that is in series with the string of all the magnets in the ring. Since the reference magnet is not in the ring the search coil can be placed in the same volume of the magnet aperture that the beam occupies. This constitutes a very good sample of the average dipole magnetic field that the beam feels. The absolute accuracy of the Gauss Clock is better than $0.05 \%$.

From the measurement of the average magnetic field the average momentum of the beam can be calculated, according to,

$$
c P=0.29979 q B \rho
$$

where $\quad \rho$ is dipole magnet bending radius, meters;

$B$ is dipole magnetic field, Tesla;

$\mathrm{q}$ is the beam charge;

$\mathrm{cP}$ is beam momentum, $\mathrm{GeV}$.

The momentum determines the speed, through,

$$
\beta=\frac{1}{\sqrt{1+\left(E_{o} / c P\right)^{2}}}
$$

where $\quad E_{0}$ is beam rest energy.

The path length of the orbit then determines the revolution frequency. The path length, $C$, is parameterized by the effective radius of the orbit, $R ; C=2 \pi R$. To a very good approximation $R$ equals the physical average radius of the orbit, even though the orbit is not strictly a circle. The inaccuracy of the approximation is compensated in the running machine by the radial position feedback loop.

The radius of the orbit, $R$, and the Gauss Clock calibration coefficient, $k$, are not single numbers, however. They are functions of the magnetic field itself, $\mathrm{R}(\mathrm{B})$, and $\mathrm{k}(\mathrm{B})$. In this way one anticipates the desire to change the orbit radius during the acceleration cycle, and allows for the compensation of non-linearities in the Gauss Clock calibration. The functions, $R(B)$ and $k(B)$, are created via the interactive graphics function editor program. Figure 2.1 is sample of computer screen used to specify the functions in the rf Beam Control program. 


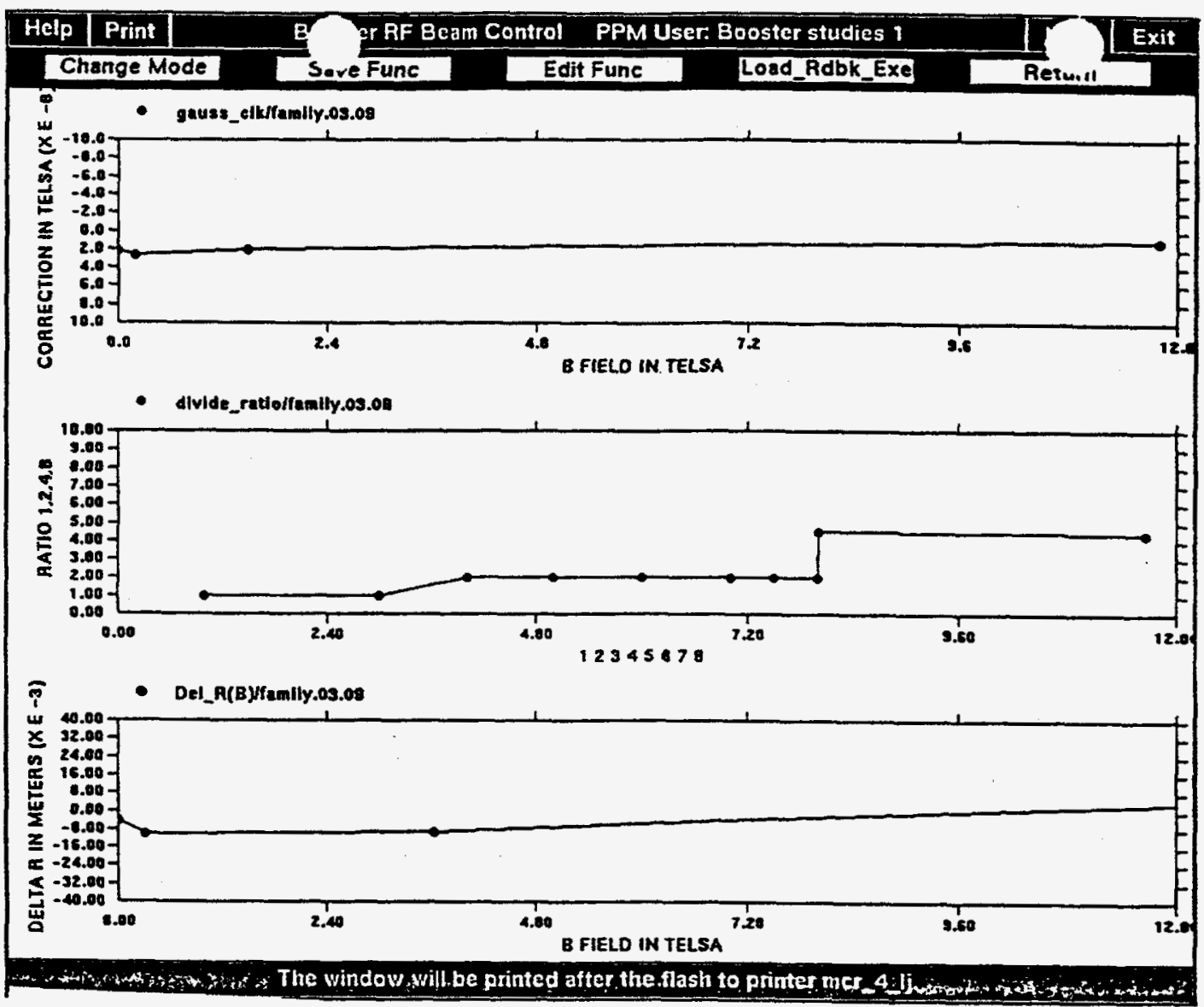

Figure 2.1. Sample of the display of the interactive computer program that is used to specify the radius, $R(B)$, Gauss Clock calibration constant, $k(B)$, and divide ratio as functions of magnetic field. 
The functions are used, off-line, to calculate a table of the revolution frequency as a function of magnetic field. The table is calculated to an accuracy of 24 bits and loaded into a Random $\underline{\text { Access }}$ Memory. As the magnetic field goes through its cycle the Gauss Clock sends a pulse every time the field increases (upcounts) or decreases (down-counts) by 0.1 Gauss. The up-counts and down-counts are used to increment and decrement a pointer to the RAM locations which store the revolution frequencies. This on-line tracking of changes in the magnetic field keeps the frequency program within a few kiloHertz or the actual beam frequency.

When the table is calculated it is assumed that location zero corresponds to zero magnetic field. This means that some locations in the table are never addressed, but on the other hand, no assumptions need to be made when the table is calculated about the value of the magnetic field when the beam is injected.

Two run-time adjustments are provided for making small corrections to the Gauss Clock calibration. A rate multiplier scales the frequency of pulses from the Gauss Clock before they increment(decrement) the pointer to the RAM. It is a 12-bit device, of which the 8 least significant bits are adjusted, giving a range from one to $\left(2^{12}-2^{8}\right) / 2^{12}=0.9375$. The rate multiplier is a pulse swallower that spaces out the swallowed pulses (up to 256 out of 4096 ) to minimize the effect on the duty factor of the pulse train. This effectively modifies the average value of the Gauss Clock calibration coefficient, $\mathrm{k}$.

The second run-time adjustment changes the effective zero of the Gauss Clock calibration curve. Sine the Gauss Clock is an integrating device (incrementing and decrementing a pointer), it must be re-zeroed periodically. This is done by down loading a pre-set value to the RAM pointer at the beginning of each machine cycle. The Gauss Clock pulses are not allowed to increment (decrement) the RAM pointer until at trigger (RF_TRACK) arrives from the machine absolute magnetic field measuring system (Ḡauss Time Line), indicating that the magnetic field equals the value pre-set in the pointer register. After that the pointer will follow changes in the magnetic field until the end of the machine cycle. By changing the pre-set value of the pointer ("B $B_{\text {START }}$ ") one effectively changes the zero value of the Gauss Clock calibration curve.

The nominal value of the Gauss Clock calibration is 0.1 Gauss per pulse. The RAM has $64 \mathrm{~K}$ locations giving a range of $6.4 \mathrm{KGauss}$ in the frequency program table. To go beyond 6.4 kGauss the Gauss Clock pulse train can be divided by 2,4 , or 8 . The divide ratio is specified before the table is calculated with the graphic function shown in Figure 2.1. When the table is calculated the incremental value of magnetic field for each location is calculated from $k(B) x$ (divide ratio), and the divide ratio is stored in two bits at that location. At run-time the two bits are used to control a divider circuit on the incoming Gauss Clock. The divide ratio can be specified in eight equal regions in the range of the magnetic field. The maximum magnetic field is calculated from the maximum expected beam momentum, an input parameter to the frequency program calculation code. 


\section{$2.2 \quad$ RF Frequency Generation}

The revolution frequency is a fundamental property of the beam, but the rf frequency can be chosen to accommodate the capabilities of the high level rf system by choosing the harmonic number. In fact the system provides for the possibility of two different harmonic numbers operating simultaneously. This is useful for various if gymnastics such as bunch coalescing or bucket shape manipulations. Two Direct Digital Synthesizers can generate two RF frequency programs from the same revolution frequency table with different harmonic numbers. Two four-byte registers hold up to four harmonic numbers each, these can be selected during the cycle by timing triggers. The revolution frequency data are multiplied by the harmonic numbers ( 8 bits) to produce the rf frequency data which are sent to the two synthesizers. The synthesizers are controlled with a 24 bit word, giving a frequency resolution of $3.75 \mathrm{~Hz}$.

\subsection{Cavity Band Control}

The high level rf system comprises two pairs of cavities, operating in two frequency bands, 0.6 to 3.0 and 2.3 to $5.0 \mathrm{MHz}$. As the rf frequency crosses from one band to the other, one pair of cavities must be ramped down in voltage while the other is ramped up. This ramping is naturally a function of frequency, not of time, because the frequency is determined by the magnetic field whose time dependence is arbitrary. Data from the frequency program is used to control the voltage of each cavity.

For each cavity there is a look-up table that is addressed with the 12 mostsignificant bits of the rf frequency data. The 8-bit words at each location are sent to the Cavity Level Control circuit where the are used in a multiplying DAC to scale the Voltage Command function. The Voltage Command function specifies the desired total rf voltage supplied by all cavities and is a function of time. The scaling coefficients stored in the table are constrained to sum to one so that the sum of all cavity voltages equals the Voltage Command function.

$$
V_{i}=a_{i}(f) V_{C M D}
$$

where $V_{i}$ is the AGC reference for cavity $i$ and $a_{i}(f)$ is the band control coefficient.

$$
\sum_{i=1}^{4} a_{i}(f)=1
$$


In this way the operator need only specify the desired rf voltage as function of time and the circuitry automatically allocates the proper fraction to the total among the individual cavities as the frequency changes.

The eight most-significant bits of the rf frequency word are sent to the Cavity Level Controller where they address the frequency coordinate of cavity personality PROM (see Section 1.4.2).

\subsection{The Local Oscillator Frequency}

Before the rf frequency data are sent to the synthesizers a fixed number corresponding to $10.700000 \mathrm{MHz}$ is added to the digital word. This causes the synthesizers outputs to be always $10.7 \mathrm{MHz}$ above the actual if frequency program. The signal then serves as the local oscillator in the hetrodyning system described below in Section 3. 


\section{HETERODYNE SYSTEM}

\subsection{Motivation}

The successful operation of the beam control system depends on effectively damping the coherent synchrotron oscillations. This requires a high quality measure of the phase of the beam bunches with respect to the total rf voltage. Since the details of the bunch shape can vary greatly during acceleration, the meaning of the "phase" of bunch signal can be ambiguous. Operationally, some definition of the phase of the beam must be taken, and some definitions are more effective than others. For example, such definitions as the time of the peak of the pulse or the time of the zero crossing of the derivative of the pulse can change their significance as the bunch shape changes.

To arrive at a useful definition the first point to realize is that there must necessarily be some delay. It is impossible to make a signal that indicates promptly when the average of the bunch is occurring because the average is determined in part by signal from the bunch that occurs after the average. Delay on this time scale $\left(1 / \mathrm{f}_{\mathrm{rf}}\right)$, however, is not detrimental to the capabilities of the phase feedback loop. The beam cannot respond faster than a time on the order of the synchrotron period.

The definition used in this system is, the phase of the beam is the phase of the single Fourier component of the bunch signal at the fundamental if frequency. Since the Fourier spectrum of the bunch signal is a discrete-line spectrum, this definition amounts to filtering the spectrum such that only one line remains and then measuring the phase of the resulting pure sine wave (with respect to the phase of the pure sine wave of the cavities' voltage). The bandwidth of the filter used determines the effective averaging time over which the phase measurement is made. As long as only one line of the spectrum is selected the details of the bunch shape will not affect the phase measurement.

The choice of the filter bandwidth (averaging time) depends on the revolution frequency and the bunch pattern in the ring. If the ring is full of evenly-spaced equal bunches then the spectrum will have lines only at the harmonics of the rf frequency. If the bunch populations are not equal or not all rf buckets are filled then the line spacing will be the revolution frequency. In the Booster we have a wide range of revolution frequencies ( 60 to $930 \mathrm{kHz}$ for Gold ions and 800 to $1370 \mathrm{kHz}$ for protons) but the line spacing in the beam spectra are not very different. This is because for heavy ions the ring is always full of equal bunches (line spacing $=\mathrm{f}_{\mathrm{rf}}>800 \mathrm{kHz}$ ) while for protons we may have one, two, or three bunches but the revolution frequency is $>800 \mathrm{kHz}$. For this reason we take a filter bandwidth of $300 \mathrm{kHz}$ for all beams. This bandwidth brings a averaging time on the order of 3 microseconds and is a significant component of the total delay in the phase feedback loop (see below, Section 4.1.2). 
The motivation for using the heterodyne technique is so that a simple filter with fixed center frequency can be used, even though the rf frequency sweeps over a wide range. The heterodyne technique continuously shifts the beam signal and the signal from the cavities into a fixed-frequency band, centered at the filter center frequency.

\subsection{Hardware}

3.2.1 Principle of Operation. Figure 3.1 shows the principle of operation of the heterodyne system. A Local Oscillator signal is generated in a direct digital synthesizer with frequency $10.70000 \mathrm{MHz}$ above the frequency given by the frequency program (see above, Section 2.4). A signal from a Voltage Controlled Oscillator whose nominal frequency is $10.70 \mathrm{MHz}$ is subtracted from the LO signal in an "output mixer".

The resulting frequency is the $\mathrm{rf}$ frequency which drives the cavities. The return signal from the cavities, which monitors the actual net cavity voltage, is combined in the "input mixer" with the LO to produce the Intermediate Frequency, according to IF = LO - RF. The beam pick-up signal is mixed with the LO also to produce another signal at the IF frequency. These two IF signals are then filtered with the filters described above and their relative phase is measured with the phase detector working at $10.7 \mathrm{MHz}$.

The feedback loop of the beam control system is closed by passing the phase detector output through the appropriate loop amplifier, whose output controls the frequency of the VCO. This makes small deviations of the cavity frequency with respect to the frequency program and the instantaneous beam frequency. On the average the beam frequency equals the cavity frequency so that the two input signals to the phase detector are the same frequency.

The details of the output mixers and the input mixers are different. Their functions differ in two important ways: signal amplitude dynamic range and output signal frequency range. The signal levels of the output mixer are essentially fixed, (a linear modulator follows the output mixer to control cavity voltage). The frequency of the output signal, however, varies over a factor of ten, 0.5 to $5.0 \mathrm{MHz}$. On the other hand for the input mixer, the signal in from the beam varies over a very wide amplitude range, from up to $3 \mathrm{~V}_{\mathrm{pp}}$ to below 1 $\mathrm{mV}_{\mathrm{pp}}$, while the output frequency is essentially constant, $10.7 \mathrm{MHz}$.

3.2.2 Input Mixer. The input mixer is implemented with a diode ring doublebalanced mixer (Mini-circuits TAK-3) used in the single sideband configuration. The TAK-3 is a high level mixer with a $+17 \mathrm{dBm}$ LO drive, giving a maximum of $+14 \mathrm{dBm}$ level at the rf port. Some pre-filtering is done before the mixer to reduce harmonics of the beam and cavity signal at the intermediate frequency, 10.7 MHz. Otherwise, some fraction of the $10.7 \mathrm{MHz}$ signal would couple directly through the mixer and beat with the desired $10.7 \mathrm{MHz}$ signal to cause distortion. The unwanted sideband is $2 \mathrm{f}_{\mathrm{rf}}(>1 \mathrm{MHz})$ away and is easily rejected by the narrowband filter. 


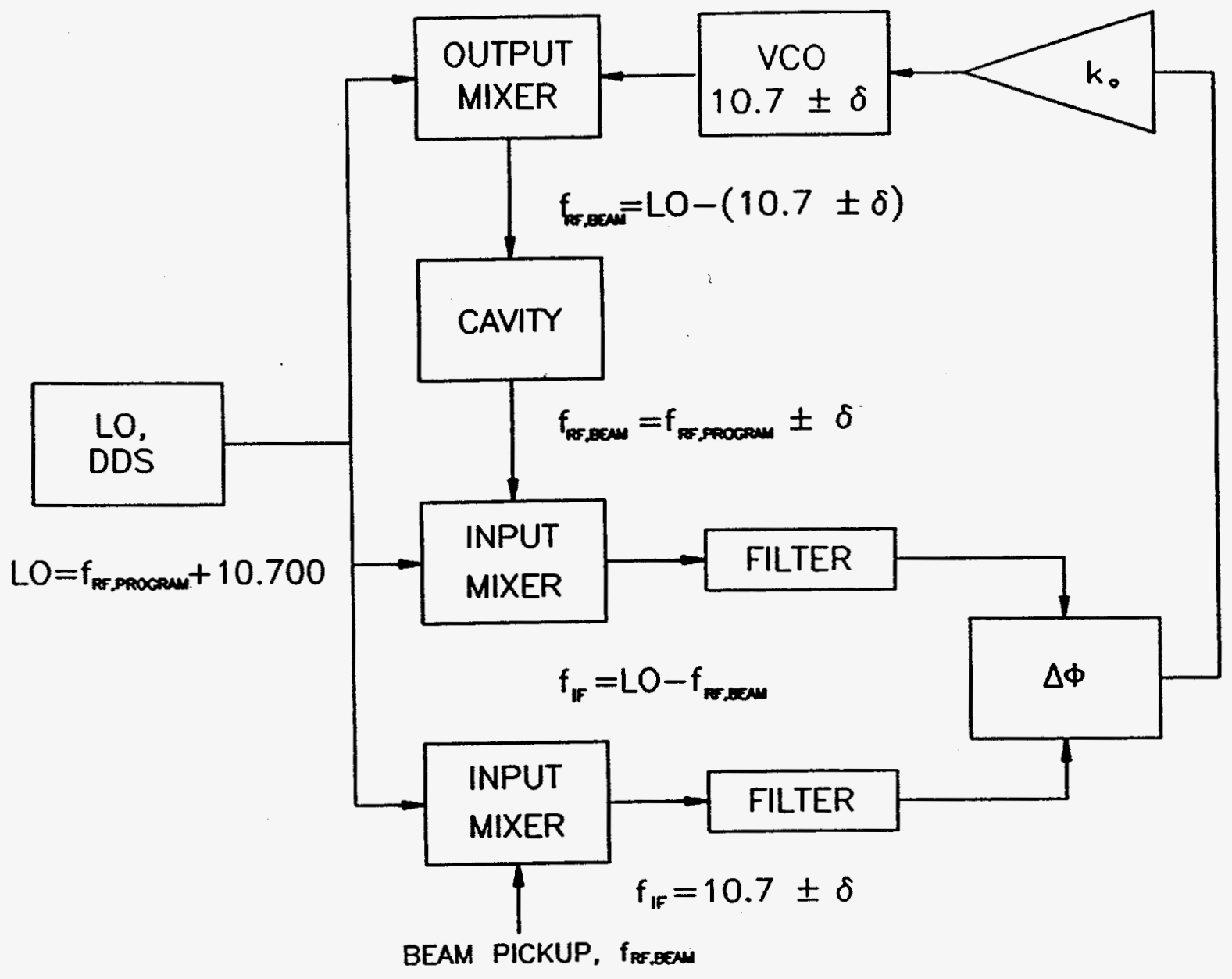

Figure 3.1. The principle of operation of the heterodyne system. This technique allows the phase detector and filters to operate at a single frequency, $10.7 \mathrm{MHz}$. LO stands for Local Oscillator. IF stands for Intermediate Frequency. DDS means Direct Digital Synthesizer. 
3.2.3 Output Mixer. For the output mixer spectral purity is important. The output frequency can be as high as $5 \mathrm{MHz}$ but the unwanted sideband can be as low a $22 \mathrm{MHz}$ (when LO $=11.2 \mathrm{MHz}$ and $\mathrm{IF}=10.7 \mathrm{MHz}$ ). A filter that strongly rejected $22 \mathrm{MHz}$, by $40 \mathrm{~dB}$ for example, would have an important effect at $5 \mathrm{MHz}$, amplitude and phase. For this reason the output mixer uses the double sideband technique implemented with active four-quadrant analog multipliers (Analog Devices AD834).

Performance of the double sideband mixing is enhanced by the capabilities of the direct digital synthesizer that generates the Local Oscillator signal. Because the LO is generated by direct synthesis it is possible to create both orthogonal components (sine and cosine). The two components remain equal in amplitude and at 90 degrees throughout the frequency range of the LO, a property very hard to achieve with analog techniques. The result of high quality LO and the linear multipliers is that the unwanted sideband is down by more than $40 \mathrm{~dB}$ and the intermodulation products are much less than double-balanced mixers would give. A final filter, lowpass with corner frequency $(3 \mathrm{~dB})$ at $10 \mathrm{MHz}$ cleans up the debris at high frequencies. Some spectra of the output signal of the output mixer are shown in Figure 3.2 One can see that the unwanted spectral lines are down by more than $50 \mathrm{~dB}$ from the desired output.

The AD834 is also used as a linear modulator in the output stage of the output mixer. The range of the modulator can be seen in Figure 3.2 to be greater than $80 \mathrm{~dB}$ at $4.1 \mathrm{MHz}$ and $60 \mathrm{~dB}$ at $0.5 \mathrm{MHz}$. Figure 3.3 illustrates the linearity of the modulator and the magnitude circuit that detects the cavity voltage.

3.2.4 Voltage Controlled Oscillator. The VCO must be fast in order that it not introduce unnecessary delays into the phase feedback loop. The unavoidable delays in the phase loop are in the order of 4 microseconds (cable length, cavity response, filter group delay). Speed of response (control bandwidth) in a VCO is a compromise with stability. The VCO used here, the Vectron Model VC372 was chosen for very high bandwidth, $>300 \mathrm{kHz}$. Figure 3.4 shows a measurement of the step response of the VCO. The rise time for a $7.2 \mathrm{kHz}$ step (large signal) is less than 1.0 microsecond. The stability, on the other hand, is not good enough to control the RF frequency at beam injection to a small fraction of the bucket height, especially for heavy ions. To retrieve stability a $10.7000 \mathrm{MHz}$ crystal oscillator is used to simulate the beam signal before injection. The phase loop then drives the VCO to exactly the crystal frequency, causing the rf frequency to follow precisely the value of the frequency program. To avoid a transient when switching from the crystal to the beam signal, a second phase detector is used and it is the output of the phase detectors that is switched into the phase loop amplifier.

3.2.5 Phase Shifters. Figure 1.1 shows two phase shifters before the phase detector, one for the stable phase angle, $\varphi_{s}$, and one for the radial loop, $\varphi_{\mathrm{r}}$. The phase shifters are situated at this point in the circuit because the IF frequency is essentially constant, $10.7 \mathrm{MHz}$, and phase shifter performance is generally better at fixed frequency. 


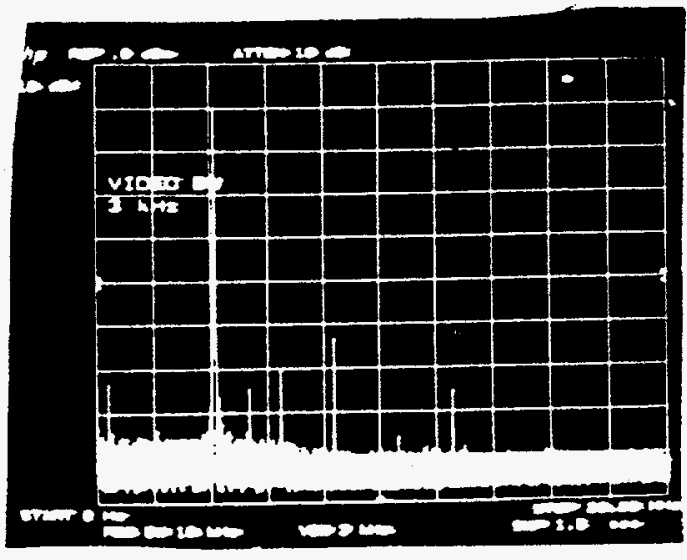

a.

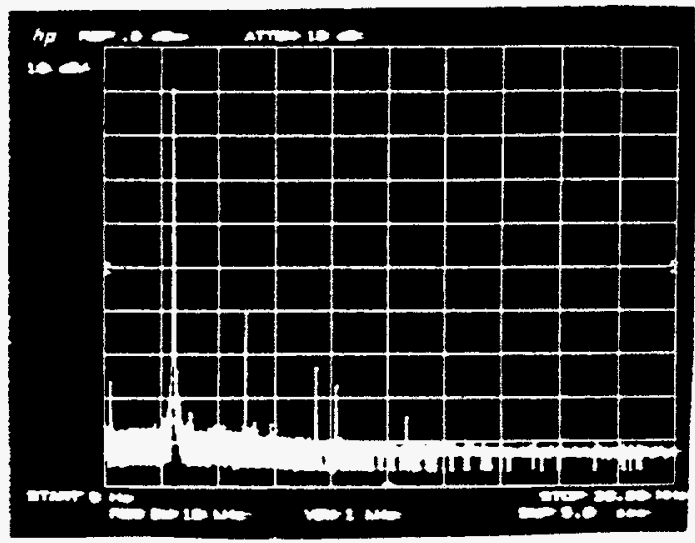

c.

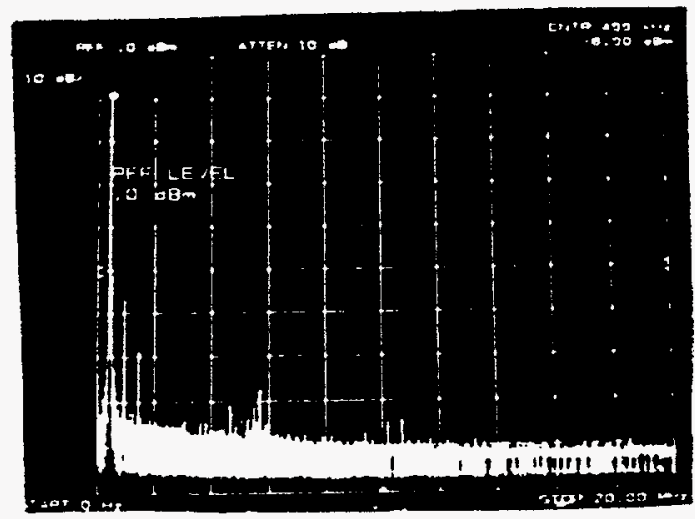

e.

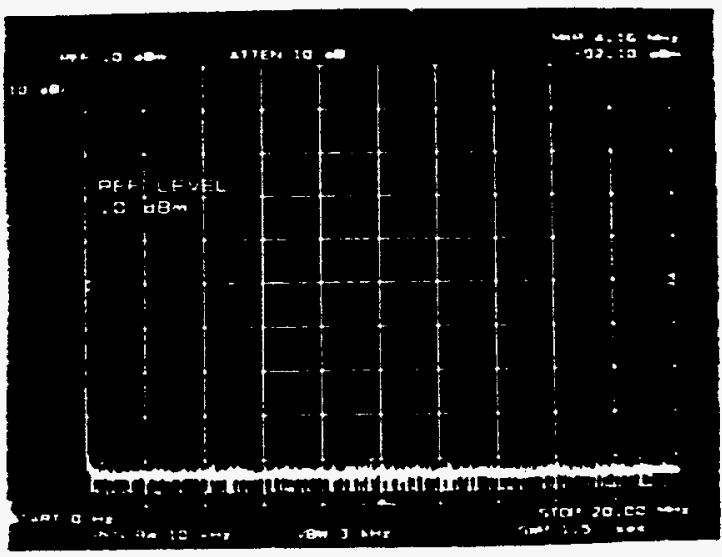

b.

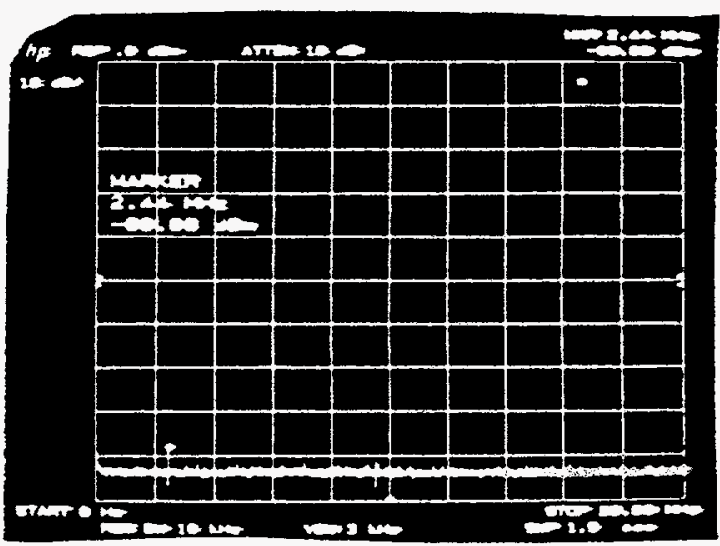

d.

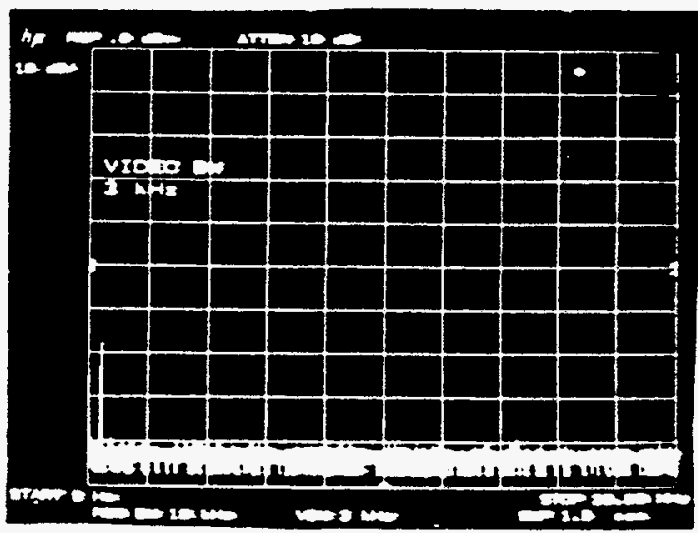

f.

Figure 3.2. Some spectra of the output signal of the Output Mixer. In a. and b. the output frequency is $4 \mathrm{MHz}$, in c. and d. it is $2.4 \mathrm{MHz}$, and in e. and $\mathrm{f}$. it is $0.5 \mathrm{MHz}$. In a., c., and e. the output is maximum and one sees that the spurious components are more than $50 \mathrm{~dB}$ below the fundamental. In $\mathrm{b}$., $\mathrm{d}$., and $\mathrm{f}$. the output in turned off by the linear modulator. One sees that the range of the modulator is more than $80,70,60 \mathrm{~dB}$ at $4,2.4$ and $0.5 \mathrm{MHz}$ respectively. 


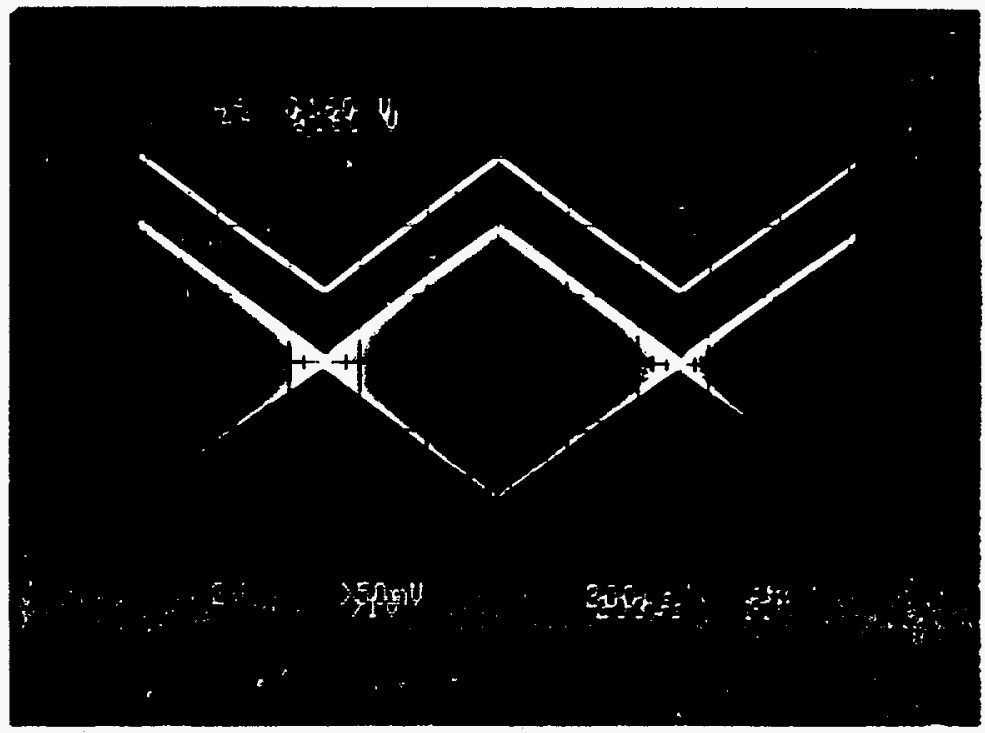

Figure 3.3. Performance of the linear modulator in the Output Mixer and the Magnitude circuit. The top trace is the control input to the modulator. The bottom trace is the if output. Superimposed on the positive envelope of the rf output is the output of the magnitude circuit. 
TVar: Frequency A

$\triangle M k r$ X: 1.216424 us

14 Jan 2023 45: 14: 35

$y: 7.2 \mathrm{kHz}$
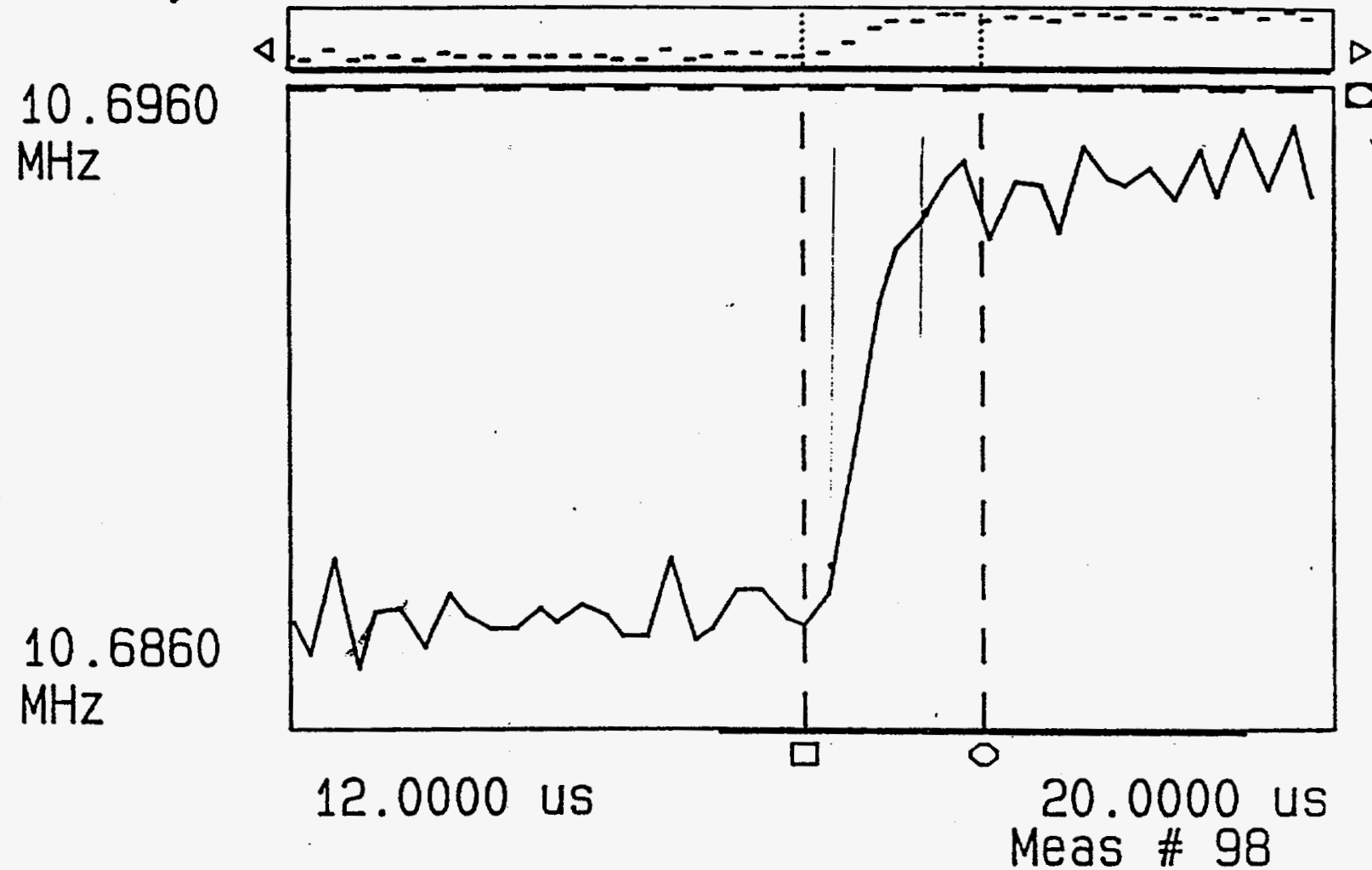

Figure 3.4. The step response of the Voltage Controlled Oscillator (VCO), measured with the HP5372A Frequency and Time Interval Analyzer. By averaging 100 measurements a frequency resolution of $1 \mathrm{kHz}$ is obtained with a time resolution of $200 \mathrm{~ns}$. This step response implies a bandwidth of over $500 \mathrm{kHz}$. 
The phase shifter modules have three modes for determining their phase shift. For mode one, a front panel pot sets the operating point, or dc phase shift. It has a range of $\pm 200^{\circ}$. The operating point adjustment is used to accommodate the rf phase difference between the longitudinal pick-up and the rf cavities.

One of the other modes can be summed with the dc phase shift, providing an additional $\pm 200^{\circ}$, so that there is always a $>360^{\circ}$ range about the operating point.

Mode two is an external voltage control with $50^{\circ} /$ Volt sensitivity and $>100 \mathrm{kHz}$ bandwidth. Conformance to linear control is $\pm 4^{\circ}$ over the whole operating range. External voltage control is used in the $\varphi_{\mathrm{r}}$ phase shifter and driven by the radial loop amplifier, see Figure 1.1.

Mode three gives a phase shift whose sine is proportional to the ratio of two inputs. The angle in this case is limited to the first and fourth quadrant and saturates to $\pm 90^{\circ}$ when the magnitude of the ratio becomes greater than one. This mode is used in the phase shifter for $\varphi_{s}$.

The phase shifter for $\varphi_{s}$ is a feedforward function. Since the phase feedback loop keeps the output of the phase detector zero, the phase between the bunch and the cavities' voltage will be the negative of the phase shift from the $\varphi_{\mathrm{s}}$ phase shifter (assume for the moment that the $\varphi_{\mathrm{r}}$ phase shifter is at zero, its nominal value). The phase shifter, therefore, generates a phase shift equal to the negative of $\varphi_{s}$. The angle is calculated with analog circuits (Analog Devices AD639 universal trigonometric converter) within the phase shifter module, according to the relation,

$$
\operatorname{Sin} \varphi_{s}=2 \pi R \rho\left(\dot{B} / V_{r f}\right)
$$

where

$\dot{B}$ is the time derivative of the average magnetic field [Tesla/second];

$\mathrm{V}$ is the magnitude of the total rf voltage per turn [Volts]; $R$ is the average orbit radius;

$\rho$ is the bending radius to the dipole magnets.

Note that neither the charge nor the mass of the particle being accelerated appear in this relation. This means that the hardware that computes the angle does not need information about species or charge state, $\mathrm{q}$, of the particle. This comes about because the relation for $\varphi_{s}$ expresses a balance between the rate of energy gain from the rf cavities,

$$
\frac{d E}{d t}=\frac{1}{T_{\text {rev }}} q V_{r f} \operatorname{Sin} \varphi_{s}
$$


and the rate of momentum gain necessary to match the increasing magnetic field strength,

$$
\frac{d P}{d t}=q \rho \frac{d B}{d t}
$$

The two are connected by the differentials of energy and momentum, $\mathrm{dE}=$ $\beta \mathrm{d}(\mathrm{cP})$. The charge cancels out and the mass is implicit in the momentum. The beam speed and revolution period are related by $T_{\text {rev }}=(2 \pi R) / \beta c$.

The $B$ signal is obtained from a single turn winding on the pole tip of the reference dipole magnet. The calibration of this signal is $0.275 \mathrm{volts} /(\mathrm{Tesla} / \mathrm{sec}-$ ond). A low-pass filter with a corner frequency of $500 \mathrm{~Hz}$ eliminates high frequency noise (presumably electric coupling between the pole winding and the main magnetic current bus).

The magnitude to the rf voltage is taken from the command to the cavity level control system. It has a calibration constant of $10 \mathrm{kV}$ per turn/Volt. The command signal is used, as opposed to a measurement of the actual magnitude of the vector sum of the cavities' voltage, because under beam-loaded conditions the angle could affect the voltage and if the voltage affected the angle then the possibility of an instability would exist. With these calibration constants the phase shifter realizes Equation 3.1 with $R=32.1 \mathrm{~m}$ and $\rho=13.7 \mathrm{~m}$.

The benefit from this feedforward function is that the other phase shifter, $\varphi_{\mathrm{r}}$, will have a small typical value. Since the value of the $\varphi_{\mathrm{r}}$ phase shifter is determined by the radial error times the gain of the radial loop, and the gain of the radial loop cannot be made arbitrarily large (see below), a small $\varphi_{\mathrm{r}}$ means small radial error.

3.2.6 LO for Radius Measurement. As mentioned above, the circuit that processes the pick-up electrode signals, to produce a measurement of the beam position, also operates at $10.7 \mathrm{MHz}$. This is done for the same reason as in the phase measurement, so that the particulars of the bunch shape do not influence the result. A narrow-band filter at $10.7 \mathrm{MHz}$ averages out the details of the bunch shape. The same LO used in the output mixers is sent to the radius processing circuits (located in the Booster tunnel) and used to convert bunch signal to the IF frequency before filtering. Pre-filtering is also used here, see Section 3.2.2 


\section{BEAM CONTROL FEEDBACK LOOPS}

\subsection{Phase Loop}

4.1.1 Principle of Operation. The function of the phase loop is to modify the dynamics of the beam response to modulations in the rf frequency and amplitude in such a way that the other loops, radius, synchronization and frequency, can perform effectively. In Section 1 we examined the beam transfer function relating variations of beam phase to variations in $\mathrm{rf}$ frequency and found that radial feedback applied directly to the rf frequency would result in an undamped type behavior. Damped behavior is obtained through the phase feedback loop and the other loops then regulate the well-damped system.

To see how damping is achieved combine Equations 1.1 and 1.2 by differentiation.

$$
\frac{d^{2} \varphi}{d t^{2}}=-\omega_{s}^{2} \varphi+\frac{d}{d t}\left(\delta \omega_{r f}\right)
$$

where $\omega_{\mathrm{s}}$ is the synchrotron frequency.

The system will be damped if the first derivative term operates on $\varphi$, which we can realize by feedback. When the if frequency deviation is made proportional to the phase, $\delta \omega_{\mathrm{rf}}=-\mathrm{K} \varphi$, then we have the equation of motion of a damped harmonic oscillator,

$$
\frac{d^{2} \varphi}{d t^{2}}+K \frac{d \varphi}{d t}+\omega_{s}^{2} \varphi=0
$$

By making $K$ large, we can control the damping rate. When $K=2 \omega_{\mathrm{s}}$ the motion is critically damped and no oscillatory behavior occurs. For the phase loop we want to make $\mathrm{K}$ as large as possible, much larger than the value for critical damping, not because this highly over-damped motion is "sluggish" but on the contrary, because it will make the value of $\varphi$ return quickly to zero after being perturbed by an input. It is important to remember that $\varphi$ is the phase of the bunch with respect to the instantaneous stable phase angle. We want the system to be "sluggish" with regard to changes in the stable phase angle or frequency, and this will be the case when $\varphi$ is forced by strong damping to be always close to zero.

Figure 1.1 shows the implementation of this concept. The phase error, $\varphi_{\mathrm{e}}$, is amplified ("K") and applied to the frequency control input of the VCO $\left(\delta \omega_{\mathrm{rf}}\right)$. The technical challenge then in making a well functioning phase loop is to make the proportionality constant, $\mathrm{K}$, as large as possible at high frequency. The practical limitations to this come from the speed of response of the rf cavities, the delays in cables to and from the tunnel, and the group delay in the narrowband 
filter of the phase measuring circuit. The actual phase detector circuit itself and the VCO have been made very much faster than these other components and do not enter into the analysis.

The design process consists of obtaining the best transfer function for the loop amplifier, $\mathrm{D}_{\varphi}(\mathrm{s})$ in Figure 1.1. The constant $\mathrm{K}$, above, is the product of $\mathrm{D}_{\varphi}(\mathrm{s})$, the VCO constant, $\mathrm{k}_{\mathrm{o}}$, and the phase detector constant, $\mathrm{k}_{\varphi}$.

4.1.2 Design of the Phase Loop. Figure 1.2 shows the phase loop regarded as a servo system. The inner loop is the phase loop with reference input $\varphi_{\mathrm{r}}$ and output $\delta \omega_{\mathrm{rf}}$. The phase detector constant $\mathrm{k}_{\varphi}$ characterizes the summing junction where the beam phase is compared to the reference phase input $\varphi_{\mathrm{r}}$. In the more literal diagram of Figure 1.1, $\varphi_{\mathrm{r}}$ is the value of the phase shifter controlled by the radial feedback amplifier.

The open loop transfer function for the system, in the Laplace transform variable, $s$, is,

$$
G(s)=k_{\varphi} k_{o} C(s) D_{\varphi}(s) B_{\varphi}(s) e^{-\tau_{d} s}
$$

where $\mathrm{C}(\mathrm{s})$ is the transfer function of the cavities' frequency modulation response;

$\tau_{\mathrm{d}}$ is the total delay;

$\mathrm{B}_{\varphi}(\mathrm{s})$ is the beam transfer function, Equation 1.3;

$\mathrm{D}_{\varphi}(\mathrm{s})$ is the transfer function of the loop amplifier (loop compensator), to be determined below;

$\mathrm{k}_{\varphi}$ is the phase detector constant, $0.5 \mathrm{Volt} / \mathrm{radian}$;

$\mathrm{k}_{\mathrm{o}}$ is the VCO constant, $2.5 \times 10^{5} \mathrm{~s}^{-1 /} /$ Volt.

We can modify the open loop transfer function by selecting the characteristics of $\mathrm{D}_{\varphi}(\mathrm{s})$. The connection between the system's behavior and $\mathrm{G}(\mathrm{s})$ can be seen by considering the closed loop transfer function,

$$
\frac{\delta \omega_{r f}(s)}{\varphi_{r}(s)}=\frac{k_{\varphi} k_{0} C(s) D_{\varphi}(s) e^{-\tau_{d} s}}{1+G(s)} .
$$

The roots of the characteristic equation,

$$
1+\mathrm{G}(\mathrm{s})=0
$$

are the poles of the closed loop system and determine the dynamics of the motion. This follows from the fact that the inverse Laplace transform of $\delta \omega_{\mathrm{rr}}(\mathrm{s}) / \varphi_{\mathrm{r}}(\mathrm{s})$ is given by the sum of the residues at these poles. 
The root locus technique is beneficial in visualizing how the poles depend on the gain of the feedback loop, that is, the magnitude of $\mathrm{K}$ in Equation 4.1. Root locus is formulated by writing $\mathrm{G}(\mathrm{s})$ as the quotient of two functions and scaled by the gain parameter, $\mathrm{g}$,

$$
G(s)=g \frac{N(s)}{D(s)} .
$$

The characteristic equation is then

$$
\mathrm{D}(\mathrm{s})+\mathrm{gN}(\mathrm{s})=0 .
$$

The roots of this equation depend on $\mathrm{g}$, and the root locus is a map of loci of the roots as the gain varies from zero to infinity. The roots will range from the poles of $G(s)(D(s)=0, g=0)$ to the zeros of $G(s)(N(s)=0, g=$ infinity).

The root loci of Figure 4.1 illustrate the importance of the cavity response, $\mathrm{C}(\mathrm{s})$, and the time delay, $\tau_{\mathrm{d}}$. In Figure 4.1(a) $\mathrm{C}(\mathrm{s})=1, \tau_{\mathrm{d}}=0$, and $\mathrm{k}_{\varphi} \mathrm{k}_{\mathbf{0}} \mathrm{D}_{\varphi}(\mathrm{s})=\mathrm{g}$.

$$
G(s)=g B_{\varphi}(s)=g \frac{s}{s^{2}+\omega_{s}^{2}} .
$$

In this case we have zeros at the origin and infinity and a pair of poles on the imaginary axis at $\pm j \omega_{s}$. Critical damping occurs when the poles coming from the $\pm j \omega_{s}$ converge on the negative real axis. As g increases the roots split, one going to the origin and the other going to negative infinity as g goes to infinity. In this over simplified case one would make $\mathrm{g}$ ( $\mathrm{K}$ above) arbitrarily large (until the model broke down due to saturation effects).

To see the significance of these two roots consider the closed loop transfer function for the frequency change resulting from a input at $\varphi_{\mathrm{r}}$.

$$
\begin{aligned}
\frac{\delta \omega_{r}(s)}{\varphi_{r}(s)} & =\frac{g}{1+g B_{\varphi}(s)} \\
& =\frac{g\left(s^{2}+\omega_{s}^{2}\right)}{s^{2}+g s+\omega_{s}^{2}}
\end{aligned} .
$$



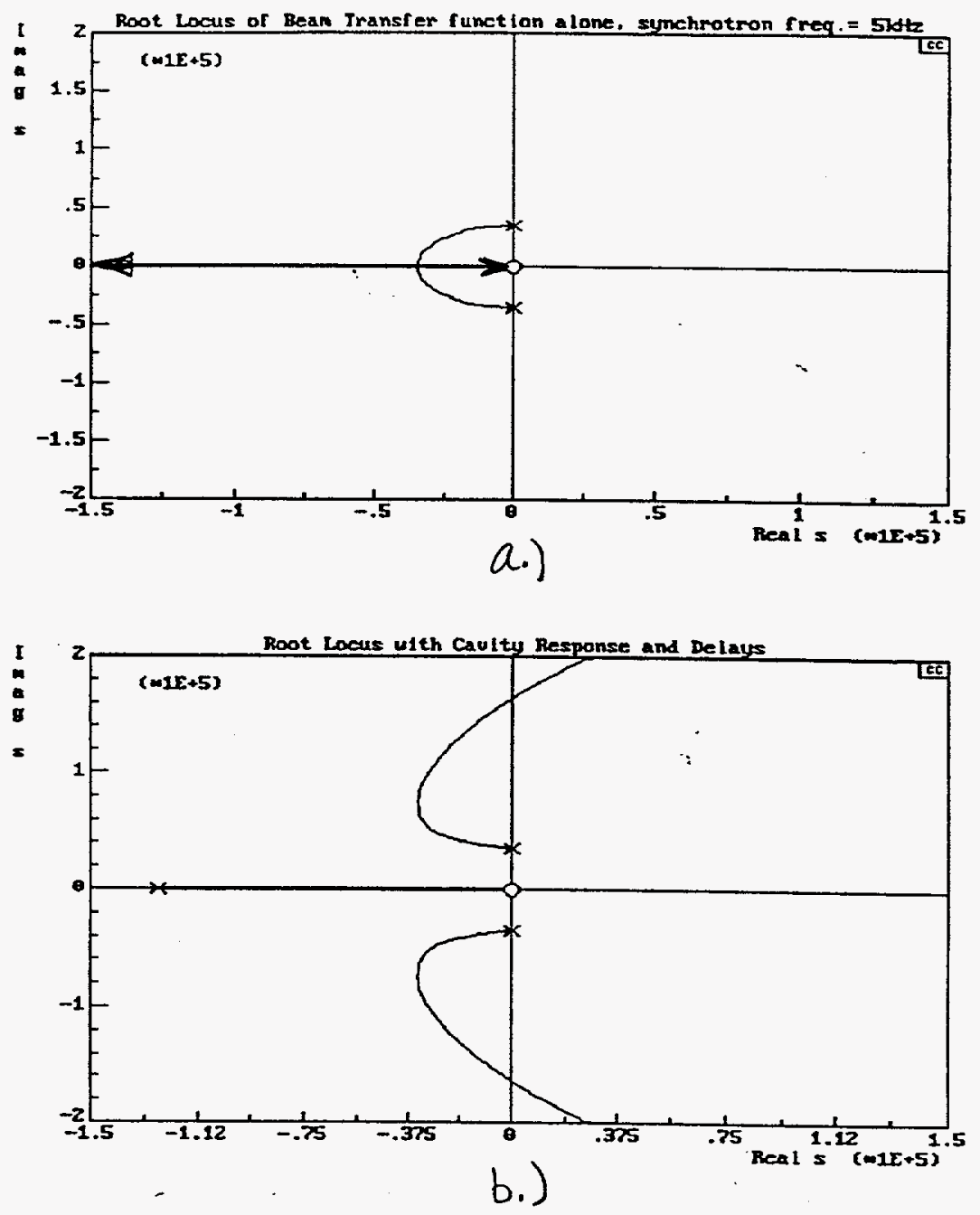

Figure 4.1. Root loci of the phase loop, a. without including cavity response and time delay, and b. cavity response and time delay included. In $b$. the poles of the synchrotron motion never reach the negative real axis. Instead, they turn and go into the right halfplane at high gain, implying instability. 
For large $\mathrm{g}$ (compared to the synchrotron frequency) the inverse Laplace transform for $\delta \omega_{\mathrm{rf}}(\mathrm{s})$ in response to a step in $\varphi_{\mathrm{r}}(\mathrm{s})=\varphi_{\mathrm{o}} / \mathrm{s}$ is

$$
\delta \omega_{r f} / \varphi_{o}=\omega_{s}^{2} t+g e^{-g t} .
$$

The first term, due to the pole near the origin, is not very interesting. It gives the linear ramp in frequency that results from a change in the energy gain per turn that occurs when the bunch moves to a new phase of the rf wave. The ramp rate depends on the synchrotron frequency only and not the gain. This slow signal will generally be the error signal to one of the other feedback loops, radius, frequency, synchronization.

The second term shows the action of the fast phase feedback. A step on the $\varphi_{\mathrm{r}}$ input will instantaneously change the rf frequency by an amount given by the forward gain and the VCO's control constant, $\delta \omega_{\mathrm{rf}}(\mathrm{o})=\mathrm{g} \varphi_{\mathrm{o}}$. The feedback will then force the rf frequency deviation back to zero with a time constant inversely proportional to the gain. This has made the rf frequency a slave to the beam, and the greater the gain the tighter the coupling between the beam frequency and the frequency of the rf cavities.

Unfortunately, neglecting the cavity response time and the time delays in the hardware is not a practical approximation.[12] Figure 4.1(b) shows the root locus when these effects are included. The model of the cavity frequency modulation response is a single pole at $s_{c}=2 \pi 20 \mathrm{kHz}$, augmented with a delay of $\tau=2.0 \mu \mathrm{s}$.

$$
C(s)=\frac{e^{-\tau s}}{\left(s / s_{c}+1\right)} .
$$

The cavities' modulation response was measured at various rf frequencies and voltage levels. The measurements were made by driving the VCO with the output of a Dynamic Signal Analyzer, HP3562A, and detecting frequency variations returning from the cavity with a fast frequency discriminator. The full cable lengths and narrowband IF filter were included in the measurement. Figure 4.2 shows a typical result, part $a$ is the magnitude and part $b$ is the phase. The frequency axis spans $125 \mathrm{~Hz}$ to $100 \mathrm{kHz}$. The magnitude plot shows the typical $6 \mathrm{~dB} /$ octave roll-off of a single pole response, with a break frequency at $20 \mathrm{kHz}$. The phase is plotted against a linear frequency axis. One can see that the phase depends linearly on frequency, in the high frequency range above $50 \mathrm{kHz}$, indicating a pure delay of $6.5 \mu \mathrm{s}$. See Section 1.4.2 for a similar discussion of the cavities' amplitude modulation response.

To resolve how much of this delay comes from the cavity and how much from the cables and filter, the cavity was jumpered out of the measurement setup. The result is shown in Figure 4.3 where the shape of the phase plot is linear over the whole frequency range. The delay in this case is $3.9 \mu \mathrm{s}$, which agrees with 
$X=100 \mathrm{kHz} \quad \Delta X=50.12 \mathrm{kHz}$

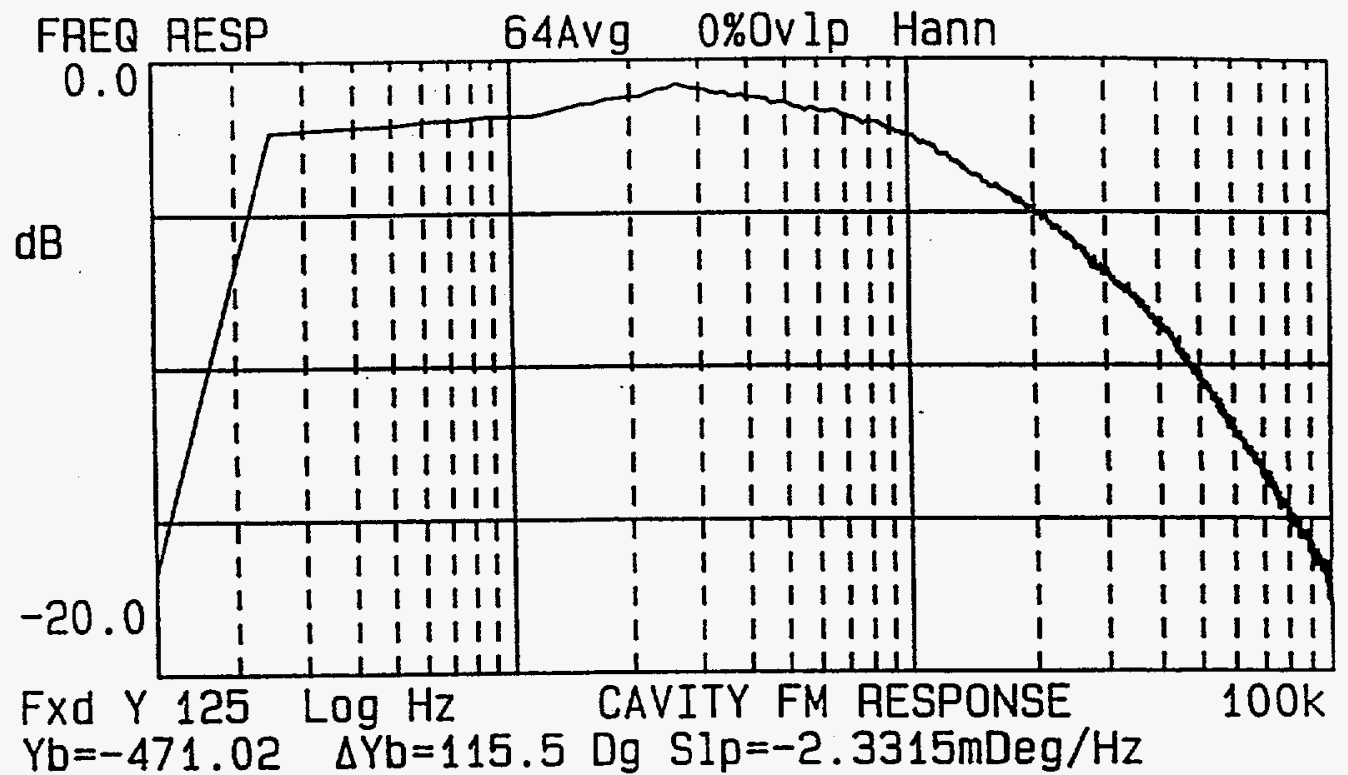

$Y b=-471.02 \quad \Delta Y b=115.5 \mathrm{Dg} S l p=-2.3315 \mathrm{mDeg} / \mathrm{Hz}$

FREQ RESP 64Avg 0\%Ovlp Hann

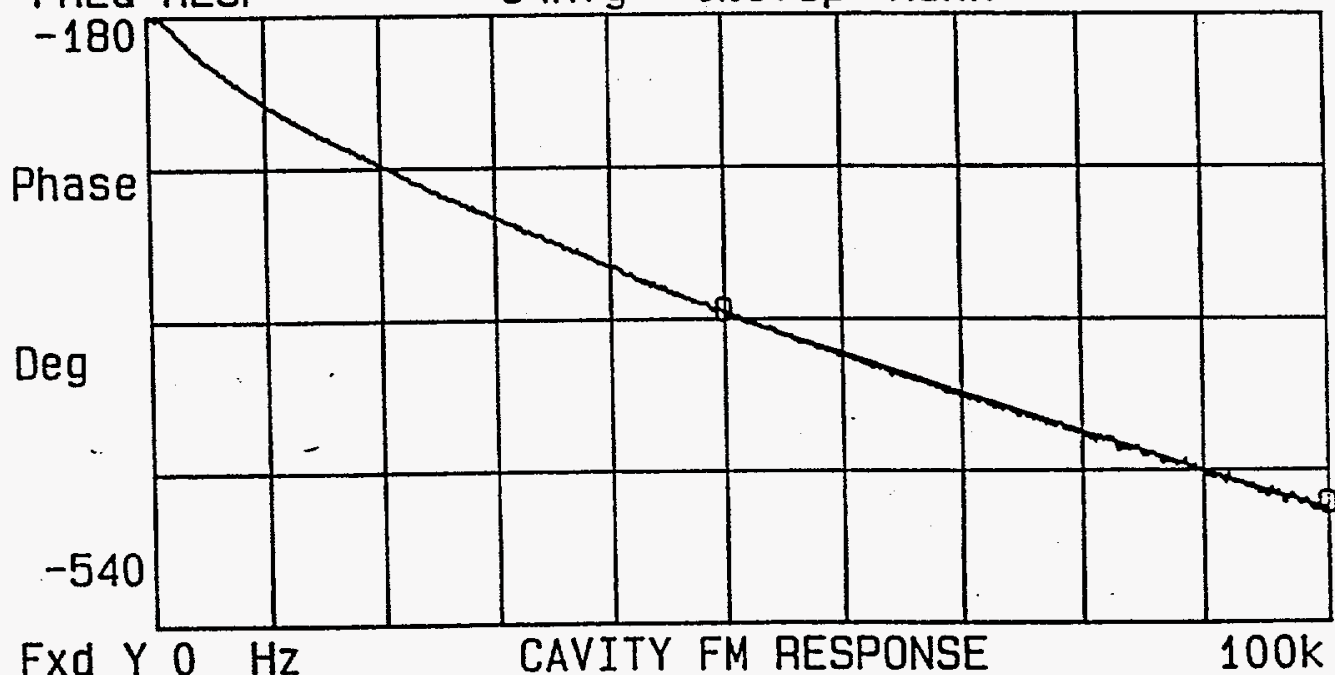

Figure 4.2. Frequency modulation response of a cavity. Top is magnitude versus log frequency, and bottom is phase versus linear frequency. The slope of the phase implies a constant delay of 6.5 microseconds. 


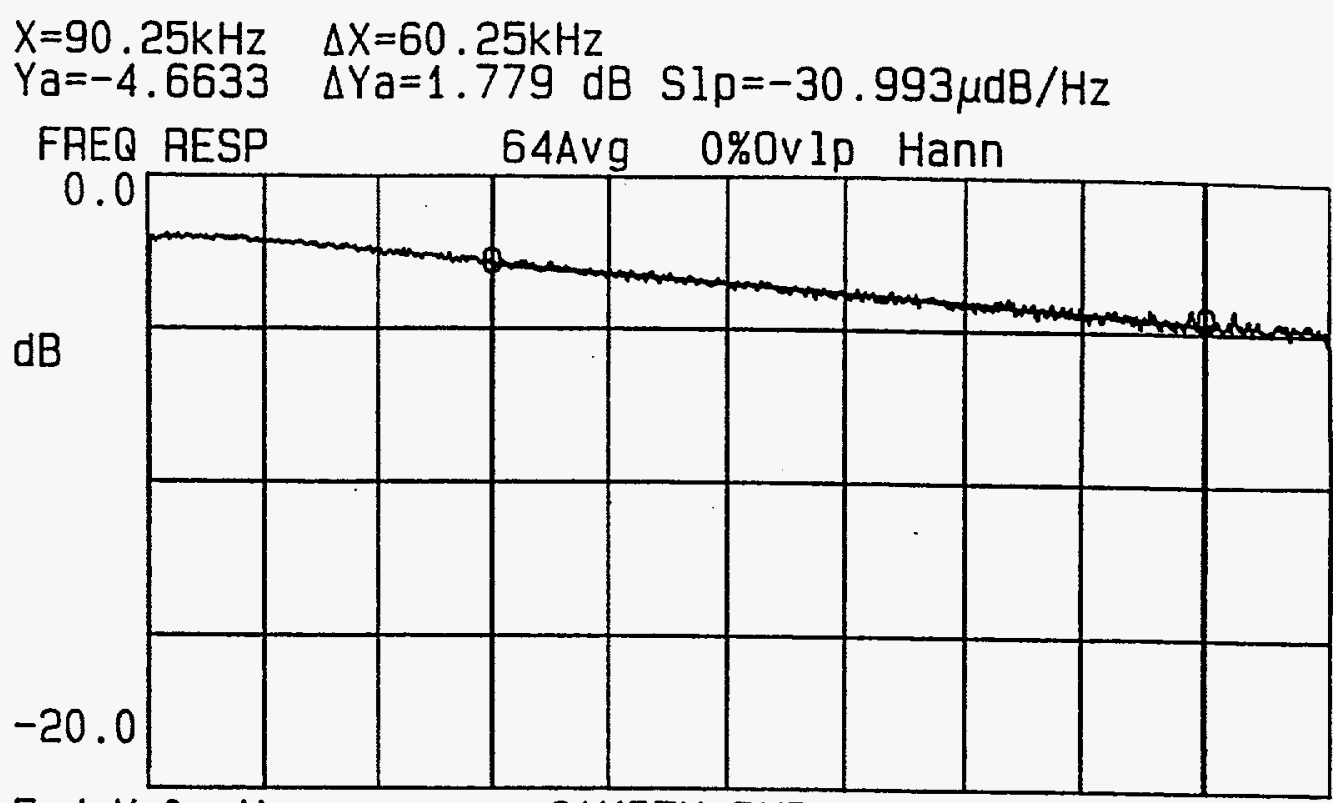

FXd Y $\mathrm{O} \mathrm{HZ}$ CAVITY BYPASSED $100 \mathrm{~K}$ $Y b=-314.43 \quad \Delta Y b=84.44 \mathrm{Dg} S \mathrm{Sl}=-1.4035 \mathrm{mDeg} / \mathrm{Hz}$

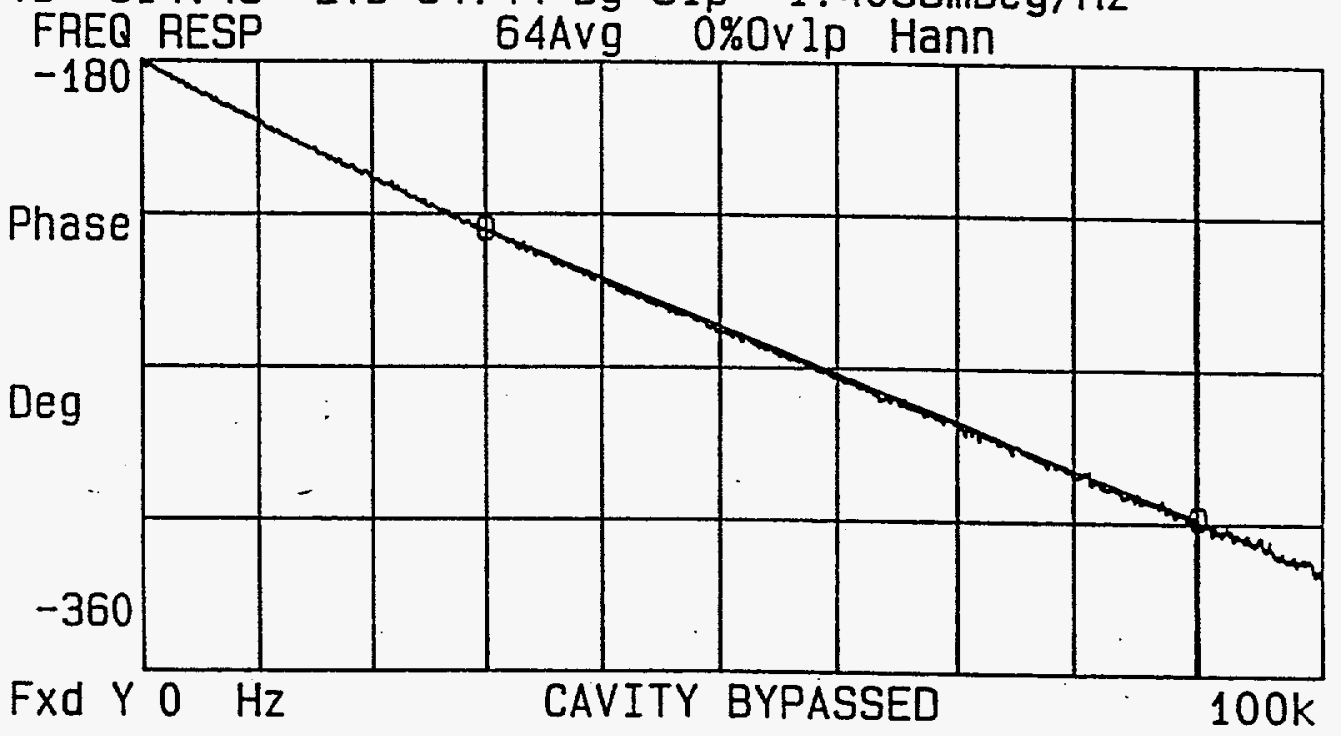

Figure 4.3. The same setup as in figure 4.2 except that the cavity is jumpered out of the measurement. The remaining delay of 3.9 microseconds is due to cable delays and filter group delay. 
group delay measurements of the filter and cable lengths. The residual delay of 2.6 microseconds is a property of the cavity. The total delay is used in the root locus analysis.

For the root locus analysis one needs an expression for the time delay with a finite number of pole and zeros. The exponential function has been approximated with a third order Padé approximation,

$$
e^{-\tau s}=\frac{1-(\tau s) / 2+(\tau s)^{2} / 10-(\tau s)^{3} / 120}{1+(\tau s) / 2+(\tau s)^{2} / 10+(\tau s)^{3} / 120} .
$$

The root locus of Figure 4.1(b)is qualitatively different from 4.1(a). Case a has no unstable roots for any value of the gain, and with sufficient gain the roots are pure real. Unfortunately in case $b$ the roots become unstable at some gain and there is no gain when all the roots are real. The property of no oscillatory response cannot be achieved.

4.1.3 Phase Loop Compensator. To achieve an acceptable phase loop response the loop amplifier, $D_{\varphi}(s)$, was built to have the PID characteristic (Proportional, Integral, Derivative). Integral improves the gain at low frequency. Proportional improves the speed of response. And derivative provides lead to offset the cavity contribution of phase shift at high frequency where loop stability is determined. Low frequency gain is important in this system because the arrangement of the phase shifters shown in Figure 1.1 requires that the phase, $\varphi_{\mathrm{e}}$, is near zero in order for the $\varphi_{\mathrm{s}}$ feedforward and radial feedback to work as designed.

Three constants, $k_{p}, \tau_{I}$, and $\tau_{D}$, characterize the PID controller. These constants correspond directly to the parameters of the op amp circuits of the loop amplifier. The transfer function is

$$
D_{\varphi}(s)=k_{P}\left(1+\frac{1}{\tau_{\mathcal{L}}}+\tau_{D^{S}} s\right) .
$$

For analysis, it is more convenient to rewrite this as product of a pole at the origin and two zeros on the negative real axis,

$$
D \phi(s)=\frac{k_{P}{ }^{\prime}}{s}\left(\tau_{D}{ }^{\prime} s+1\right)\left(s+\frac{1}{\tau_{I}^{\prime}}\right),
$$


where the parameters of the compensator are:

$$
k_{p}{ }^{\prime}=0.32 \quad \tau_{D}{ }^{\prime}=2.5 \times 10^{-5} s \quad \tau_{i}^{\prime}=3.7 \times 10^{-5} s
$$

The transformation between the two parameter sets is straightforward,

$$
k_{p}=k_{p}{ }^{\prime}\left(\frac{\tau_{D}{ }^{\prime}+\tau_{I}^{\prime}}{\tau_{I}^{\prime}}\right), \quad \frac{1}{\tau_{D}}=\frac{1}{\tau_{D}{ }^{\prime}}+\frac{1}{\tau_{I}^{\prime}}, \quad \tau_{I}=\tau_{D}{ }^{\prime}+\tau_{I}{ }^{\prime}
$$

giving hardware values:

$$
k_{p}=0.53, \quad \tau_{D}=1.5 \times 10^{-5} s, \quad \tau_{I}=6.2 \times 10^{-5} s .
$$

Written in this form, it is easy to see the effect of each of the parameters in a asymptotic Bode plot. In Figure 4.4 the open loop transfer function of beam, cavity, and delay is plotted together with the Bode plot of $\mathrm{D} \varphi(\mathrm{s})$, Equation 4.4. One sees that the pole at zero gives high low-frequency gain but a phase shift of $90^{\circ}$. In the region near the synchrotron frequency, the $90^{\circ}$ lead from the first zero cancel with this shift, and makes the magnitude of the gain frequency independent. This situation is maintained across the band of frequencies in which the synchrotron frequency may move $(1$ to $7 \mathrm{kHz}$ ). The second zero adds lead that improves the phase margin when the loop gain reaches one between 10 and $20 \mathrm{kHz}$.

With this compensator included, the root locus is plotted in Figure 4.5. The poles from the synchrotron motion converge and end up at the zeros of the PID compensator. The cavity pole and a pole from the Pade approximation to the delay converge and then move toward the positive half-plane. These roots determine the maximum gain for stability. The system is normally operated with the gain such that the poles are at $30^{\circ}$ to the imaginary axis, giving a peak overshoot in the step response of $15 \%$ and a settling time to $1 \%$ that is about 130 microseconds.

The actual hardware of the loop amplifier differs somewhat from the ideal PID equation above. The pole is not exactly at zero (the gain does not go to infinity). It is at $-1 /\left(2 \pi \tau_{\mathrm{p}}\right)$. A spurious pole and zero appear at high frequency, $-1 /\left(2 \pi \tau_{s}\right)$ and $1 /\left(2 \pi \tau_{z}\right)$, respectively, due to the op amps' finite gain-bandwidth.

Figure 4.6 is a measurement of the PID loop amplifier. These curves are fit well with two poles and three zeros, 

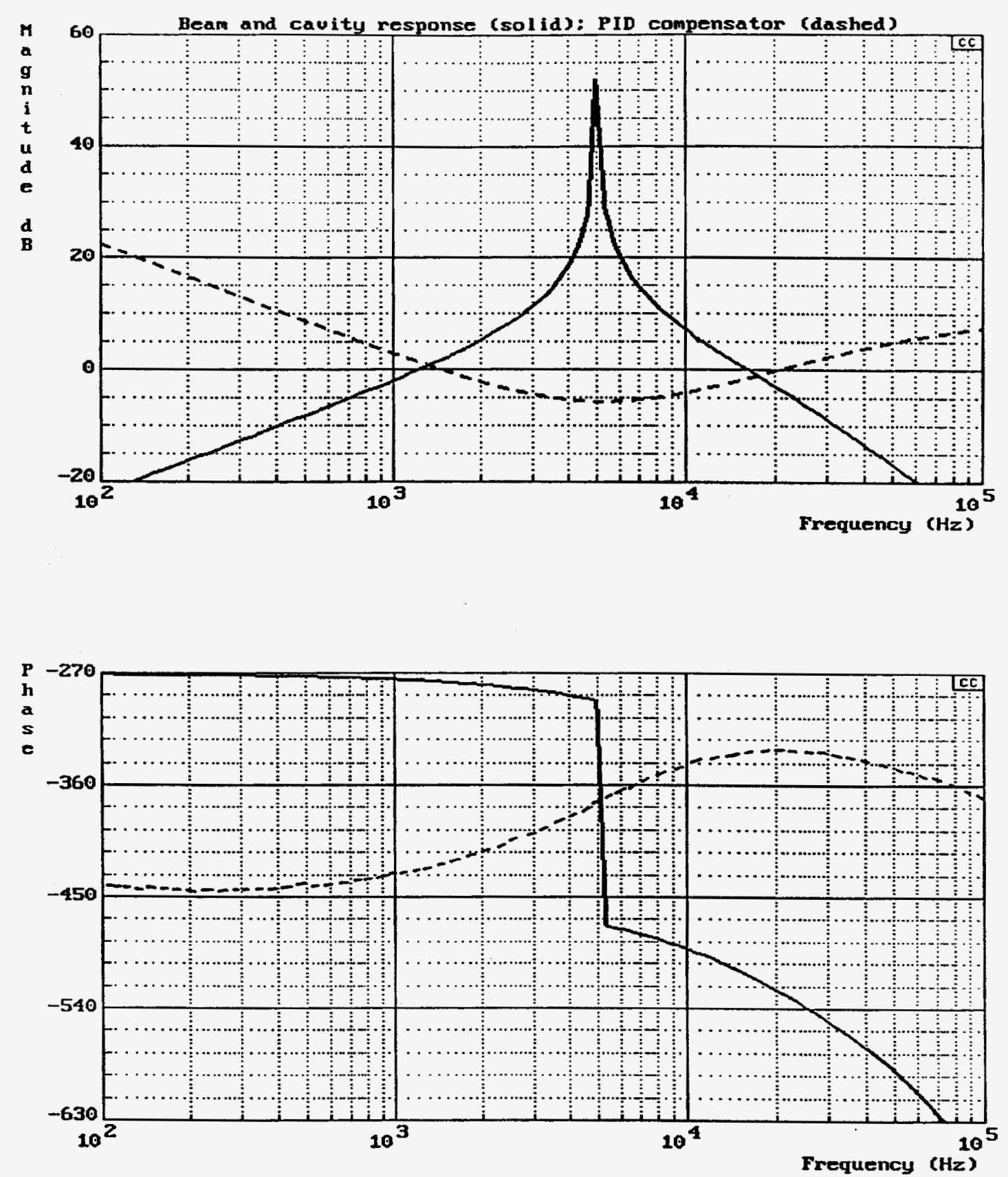

Figure 4.4 Calculation of the beam and cavity response, solid line, and the phase loop PID compensator, equation 4.4, dashed line. 


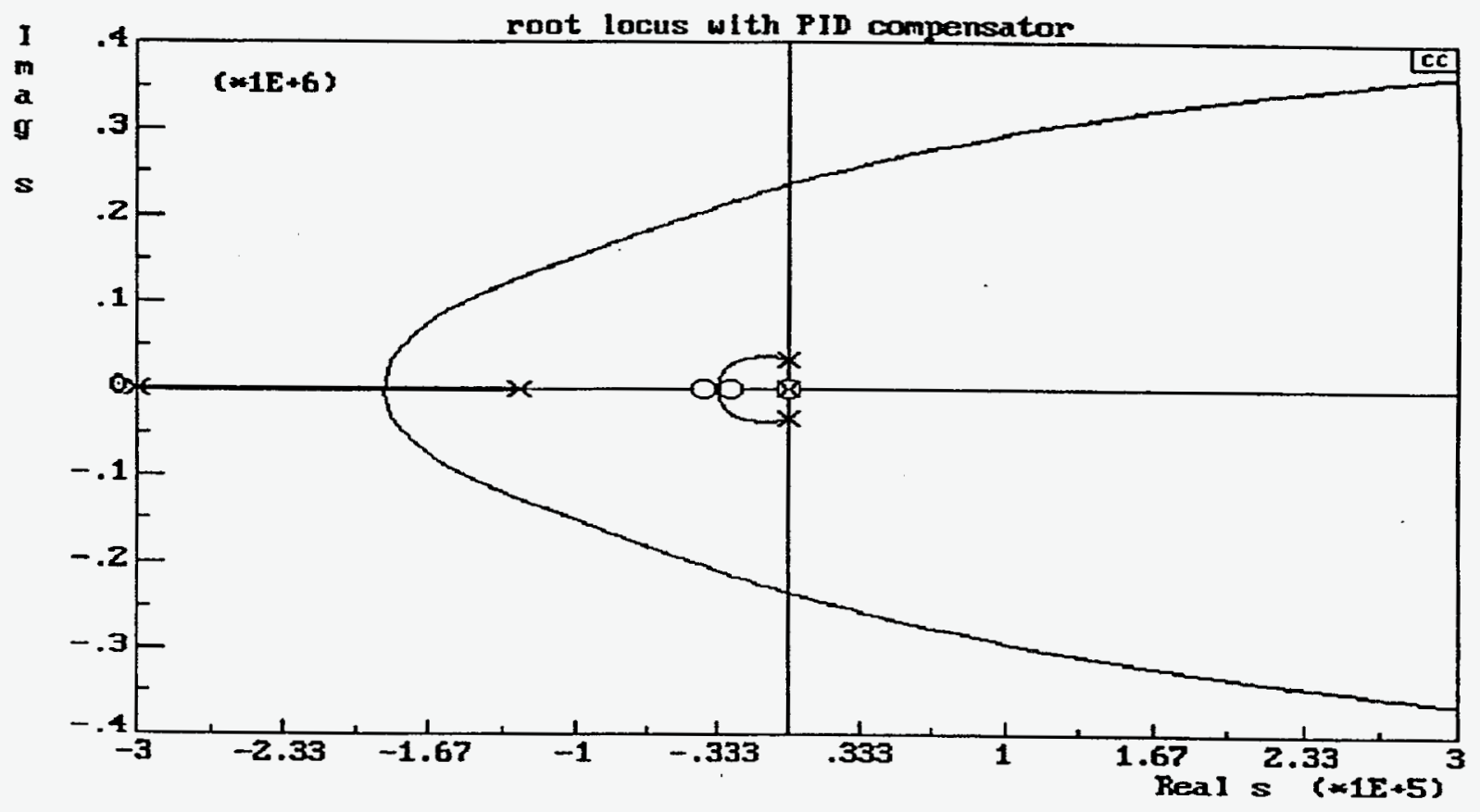

Figure 4.5. Root locus of the phase loop with the PID compensator included. 


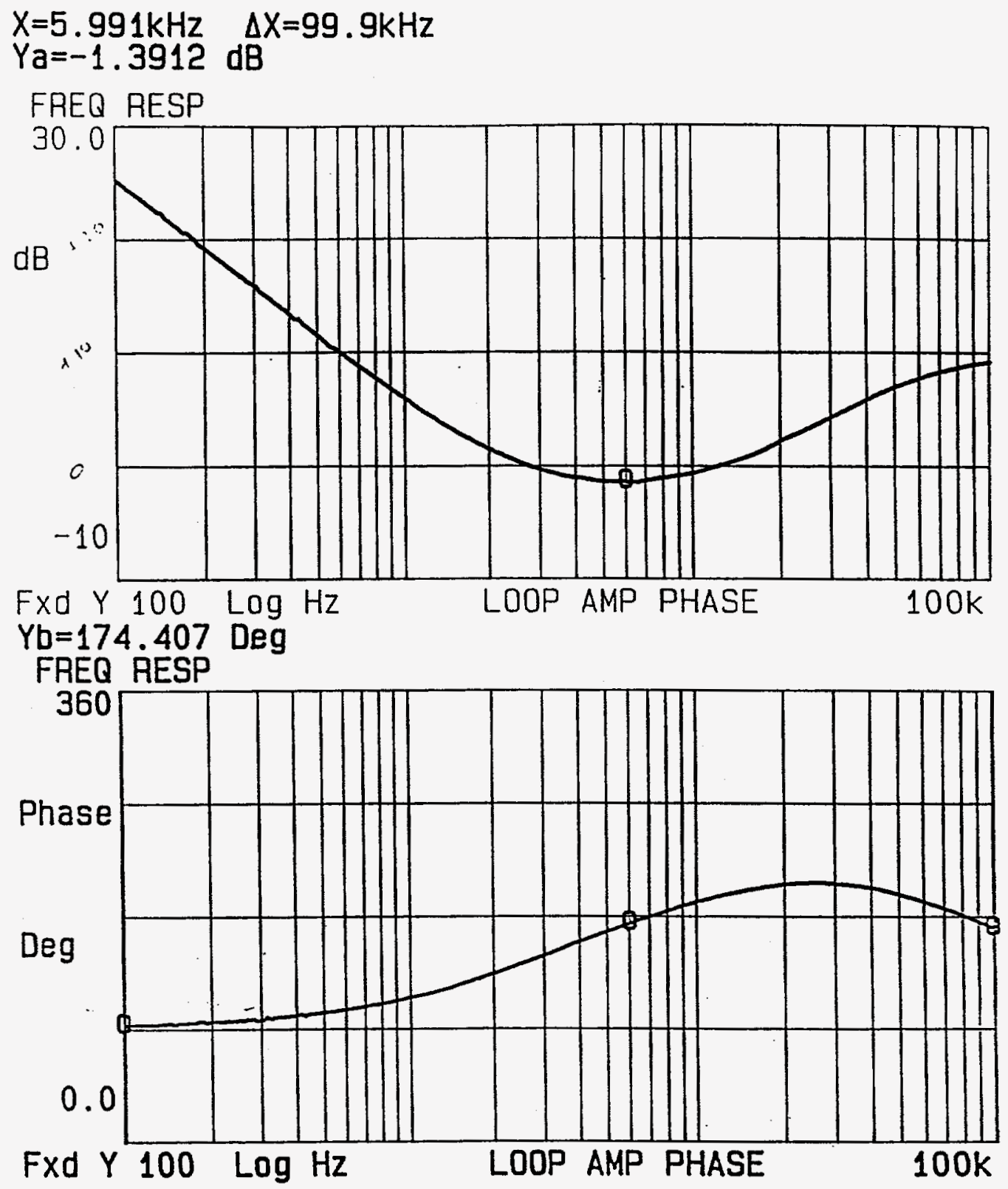

Figure 4.6. A measurement of the PID loop amplifier. The curves are well fit with two poles and three zeros, see text. 


$$
\begin{gathered}
D_{\varphi}(s)=\frac{k_{p}{ }^{\prime}\left(\tau_{D}{ }^{\prime} s+1\right)\left(s+1 / \tau_{I}{ }^{\prime}\right)\left(\tau_{z} s-1\right)}{\left(1 / \tau_{p}\right)\left(\tau_{p} s+1\right)\left(\tau_{s} s+1\right)} \\
\text { where } \begin{aligned}
\tau_{\mathrm{p}} & =0.010 \mathrm{sec} . \\
\tau_{\mathrm{s}} & =3.3 \times 10^{-6} \mathrm{sec} . \\
\tau_{\mathrm{z}} & =7.7 \times 10^{-7} \mathrm{sec} .
\end{aligned}
\end{gathered}
$$

The open loop transfer function for the phase loop with cavity response, delay, and equation 4.5 for the PID amplifier is

$$
G(s)=\frac{k_{\phi} k_{o} k_{p}{ }^{\prime}(s)\left(\tau_{D}{ }^{\prime} s+1\right)\left(s+1 / \tau_{I}{ }^{\prime}\right)\left(\tau_{z} s-1\right) e^{-\tau_{c} s}}{\left(s^{2}+\omega_{s}^{2}\right)\left(1 / \tau_{p}\right)\left(\tau_{p} s+1\right)\left(s / s_{c}+1\right)\left(\tau_{s} s+1\right)} .
$$

This is plotted in Figure 4.7 (solid line) together with Equation 4.5 for the loop amplifier (dashed line). This can be compared to a measurement of the actual phase loop operating with beam which is shown in Figure 4.8. The poles due to the synchrotron oscillation are clearly evident in the peak of the amplitude plot and the discontinuity in the phase plot. The measurement was taken with the magnetic field held constant and the radial loop open.

As an aside, this measurement can be very useful as a determination of the total effective voltage seen by the beam, because from a determination of the synchrotron frequency one can deduce the rf voltage seen by the beam. This measurement calibrates the cavity voltage monitor.

The complete closed loop response of the phase loop, considering $\varphi_{\mathrm{r}}$ as the input and $\varphi$ as the output (see Figure 1.2) is then

$$
\frac{\varphi}{\varphi_{r}}=\frac{G(s)}{1+G(s)} .
$$

This is plotted in Figure 4.9 and the measurement with beam is shown in Figure 4.10 . 



Figure 4.7. The open loop transfer function for the phase loop, with cavity response, delay, and the measured PID loop amplifier (solid curve). The response function of the measured PID loop amplifier (dashed curve). 


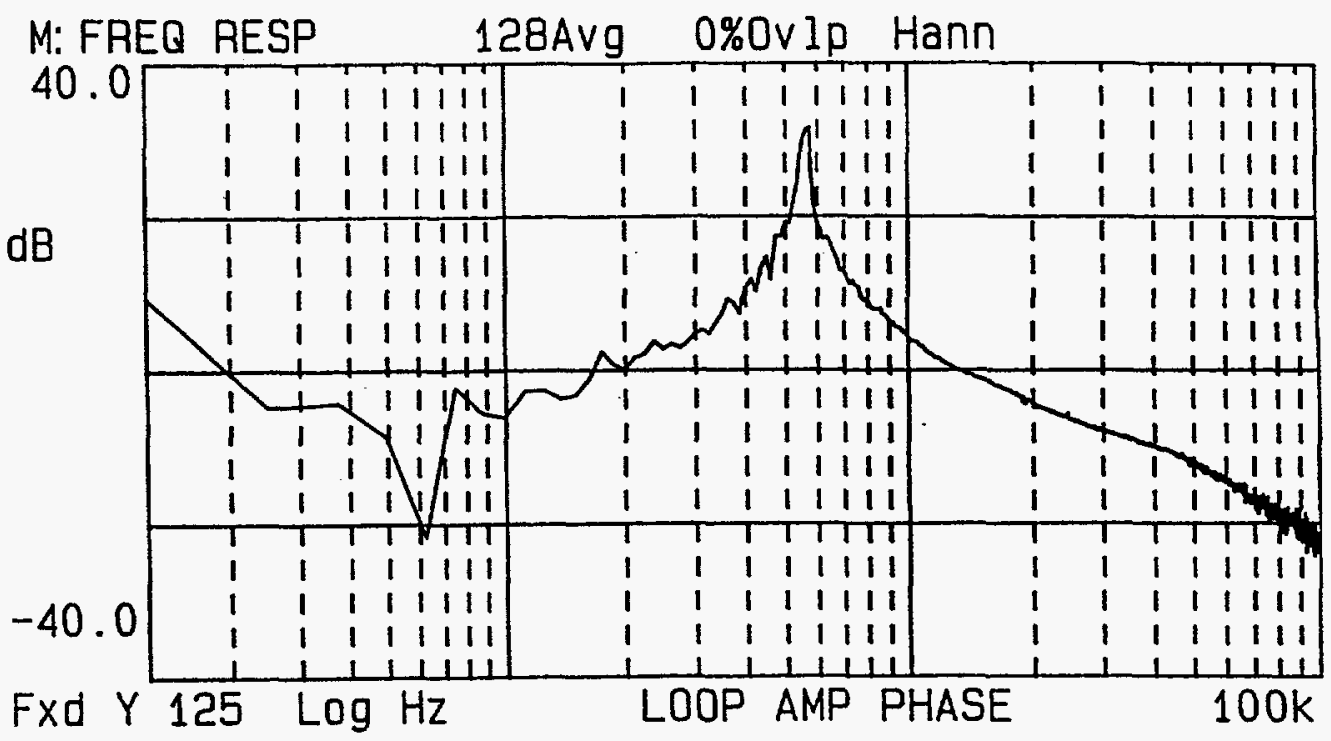

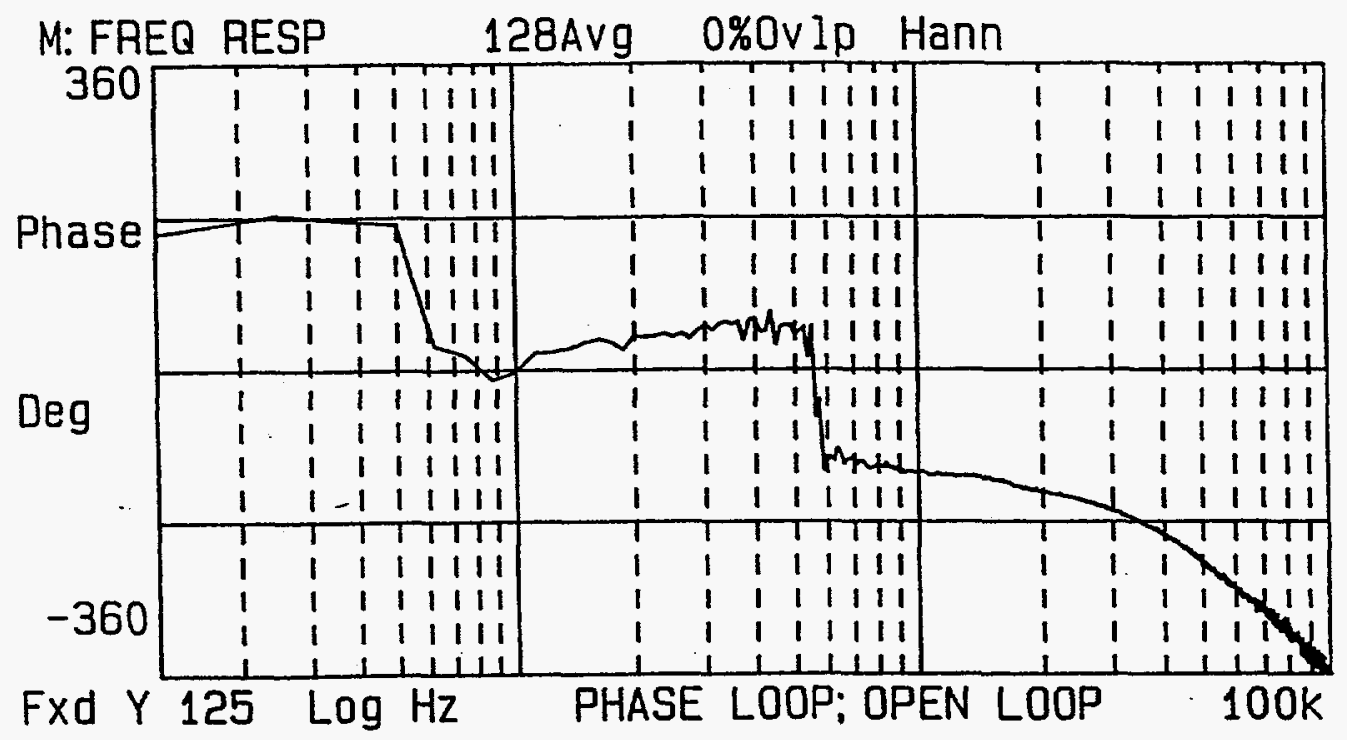

Figure 4.8. A measurement of the open loop response of the phase loop while operating with beam. 

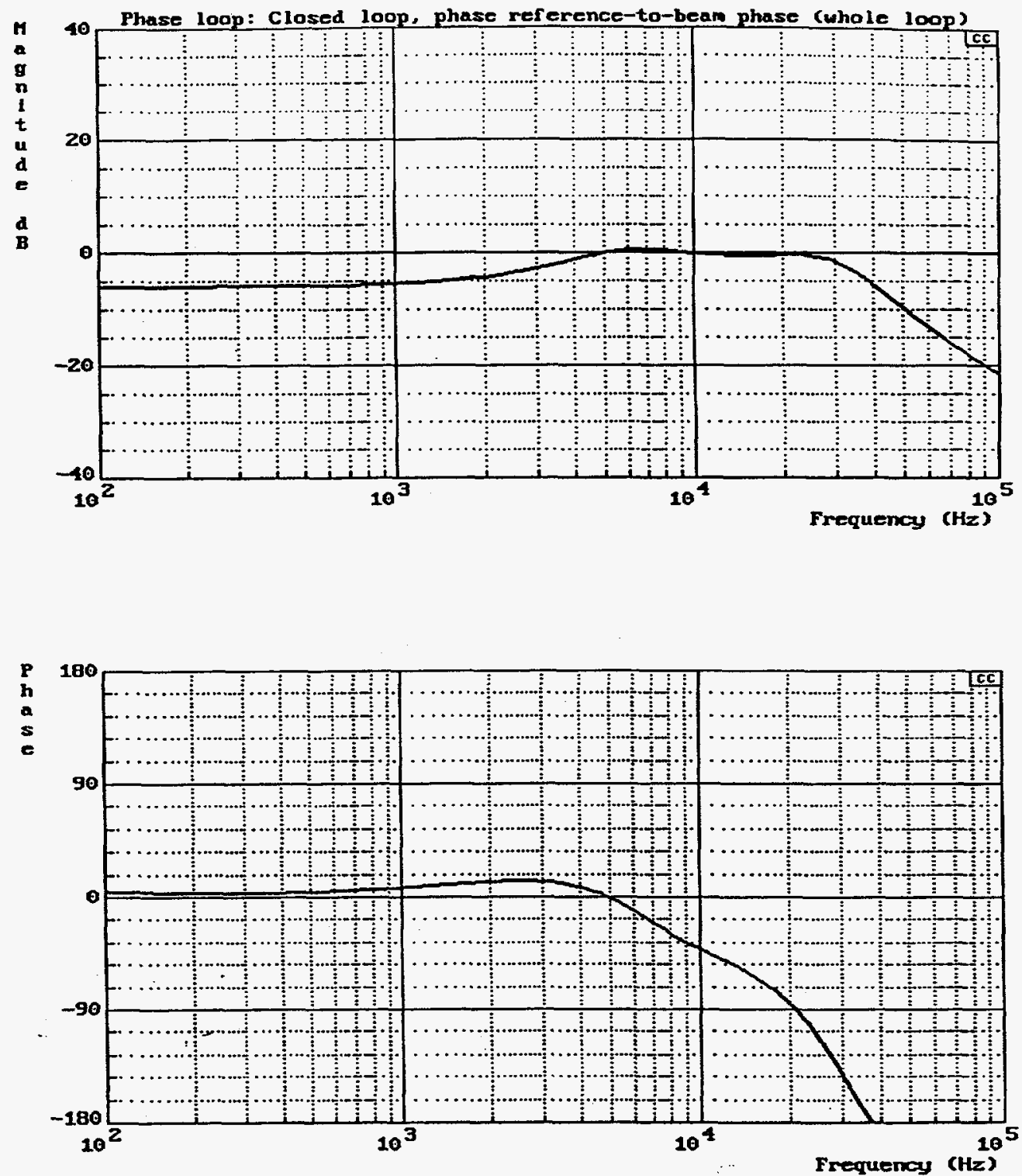

Figure 4.9. A calculation of the closed loop response of the phase loop. 


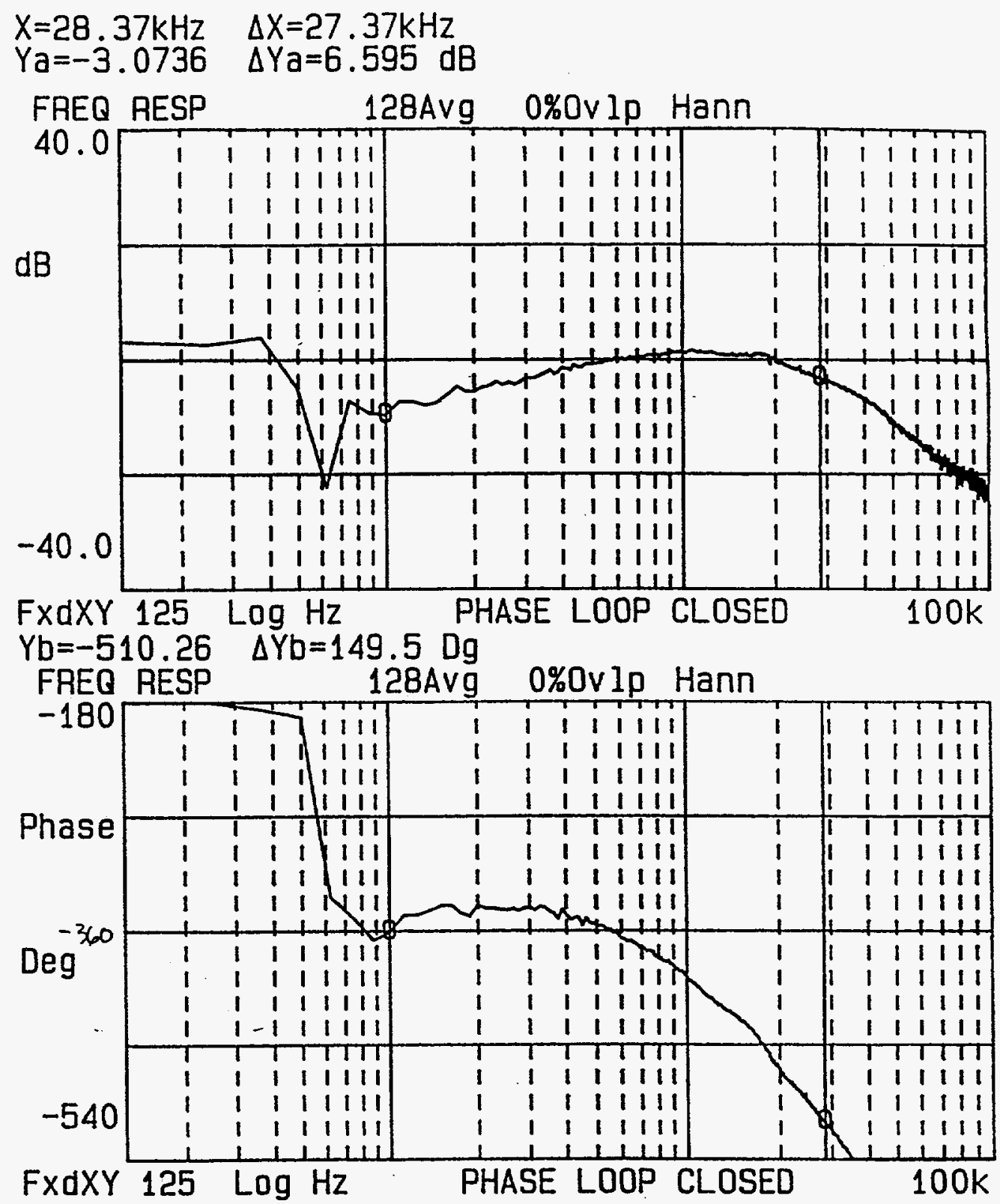

Figure 4.10. A measurement of the closed loop response of the phase loop while operating with beam. 
Although the plot of Figure 4.8 shows the open loop transfer function, the measurement was actually carried out with the loop closed, Figure 4.10. It is important that the loop remain closed so that the beam's coherent motion is well damped. Otherwise the small amplitude approximation used in this analysis may become invalid. It is possible to deduce the open loop transfer function from a measurement of the closed loop operating system. The HP3562A Dynamic Signal Analyzer is equipped with a facility to perform the mathematical operation needed,[13] and the loop amplifier was built with the required monitor port and summing junction.

The significance of the integral term in the PID compensator can be seen by considering the open loop transfer function, Equation 4.6, in the frequency decade $100 \mathrm{~Hz}$ to $1 \mathrm{kHz}$, see Figures 4.7 and 4.8. There

$$
\begin{gathered}
\tau_{p} s+1 \rightarrow \tau_{p} s \\
s+1 / \tau_{I}{ }^{\prime} \rightarrow 1 / \tau_{I}{ }^{\prime} \\
\tau_{D}{ }^{\prime}+1 \rightarrow 1 \\
\tau_{z} s-1 \rightarrow-1 \\
e^{-\tau_{d} s} \rightarrow 1 \\
s^{2}+\omega_{s}^{2} \rightarrow \omega_{s}^{2}
\end{gathered}
$$

Then

$$
G(s) \rightarrow-k_{\varphi} k_{o} k_{p}{ }^{\prime}\left(\frac{\tau_{p}}{\tau_{I}^{\prime}}\right) \frac{1}{\omega_{s}^{2}},
$$

which is a constant on the order of one $\left(=-0.84 @ \mathrm{f}_{\mathrm{s}}=5.7 \mathrm{kHz}\right.$, as shown). The beam transfer function $\mathrm{B} \varphi(\mathrm{s})$, which determines the frequency dependence at low frequencies, is essentially a differentiator at low frequencies $\left(s \ll \omega_{\mathrm{s}}\right)$. [Intuitively, the beam transfer function should behave like a differentiator because a slow ramp in rf frequency corresponds to a constant shift in beam-to-rf phase, and the output of the beam transfer function, which is phase, should go like the derivative of its input, which is rf frequency.] The pole near zero of the PID compensator has canceled the zero of the beam transfer function. The result is a loop gain that is essentially constant at low frequencies, as one sees in Figure 4.9. 
The benefit of this cancellation pertains to the radial loop. For the radial loop the closed loop response of the phase loop becomes just another block in the forward gain path. See Figure 1.2. Considering the radial loop phase shifter $\phi_{\mathrm{r}}$ as an input, the closed loop transfer function of the phase loop is

$$
\frac{\delta \omega_{r f}}{\varphi_{r}}=\frac{\left(s^{2}+\omega_{s}^{2}\right) k_{\varphi} k_{o^{\prime}} k_{p}^{\prime}\left(\tau_{D}{ }^{\prime} s+1\right)\left(s+1 / \tau_{I}{ }^{\prime}\right) e^{-\tau_{d d^{s}}}}{s\left[\left(s^{2}+\omega_{s}^{2}\right)\left(s / s_{c}+1\right)+k_{\varphi} k_{o^{\prime}} k_{p}{ }^{\prime}\left(\tau_{D}{ }^{\prime} s+1\right)\left(s+1 / \tau_{I}{ }^{\prime}\right) e^{-\tau_{d^{s}}}\right]},
$$

where Equation 4.4 has been used for $\mathrm{D} \varphi(\mathrm{s})$.

For the open loop transfer function of the radial loop, we multiply this by $B_{R}(s)$ to get

$$
\frac{\delta R}{\varphi_{r}}=\left(\frac{1}{s}\right) \frac{b k_{\varphi} k_{o} k_{p}^{\prime}\left(\tau_{D}{ }^{\prime} s+1\right)\left(s+1 / \tau_{I}^{\prime}\right) e^{-\tau_{d} s}}{\left[\left(s^{2}+\omega_{s}^{2}\right)\left(s / s_{c}+1\right)+k_{\varphi} k_{o} k_{p}^{\prime}\left(\tau_{D}{ }^{\prime} s+1\right)\left(s+1 / \tau_{I}^{\prime}\right) e^{-\tau_{d} s}\right]} .
$$

This function is plotted in Figure 4.11.

So the constant gain around the phase loop provides an integrator type response $(1 / \mathrm{s})$. The benefit of the $1 / \mathrm{s}$ behavior will become evident later when the low frequency errors of the radial loop are examined (Section 4.2.3).

The transient response of $\varphi_{\mathrm{e}}$ to an input at $\varphi_{\mathrm{r}}$ has also been calculated and is shown in Figure 4.12. A measurement of the transient response, Figure 4.13 was made by disabling the phase loop for $0.5 \mathrm{~ms}$ at injection and injecting the proton beam away from the stable phase angle. When the loop is closed it goes through its transient response. One can see the transient settles in less than one synchrotron period. 

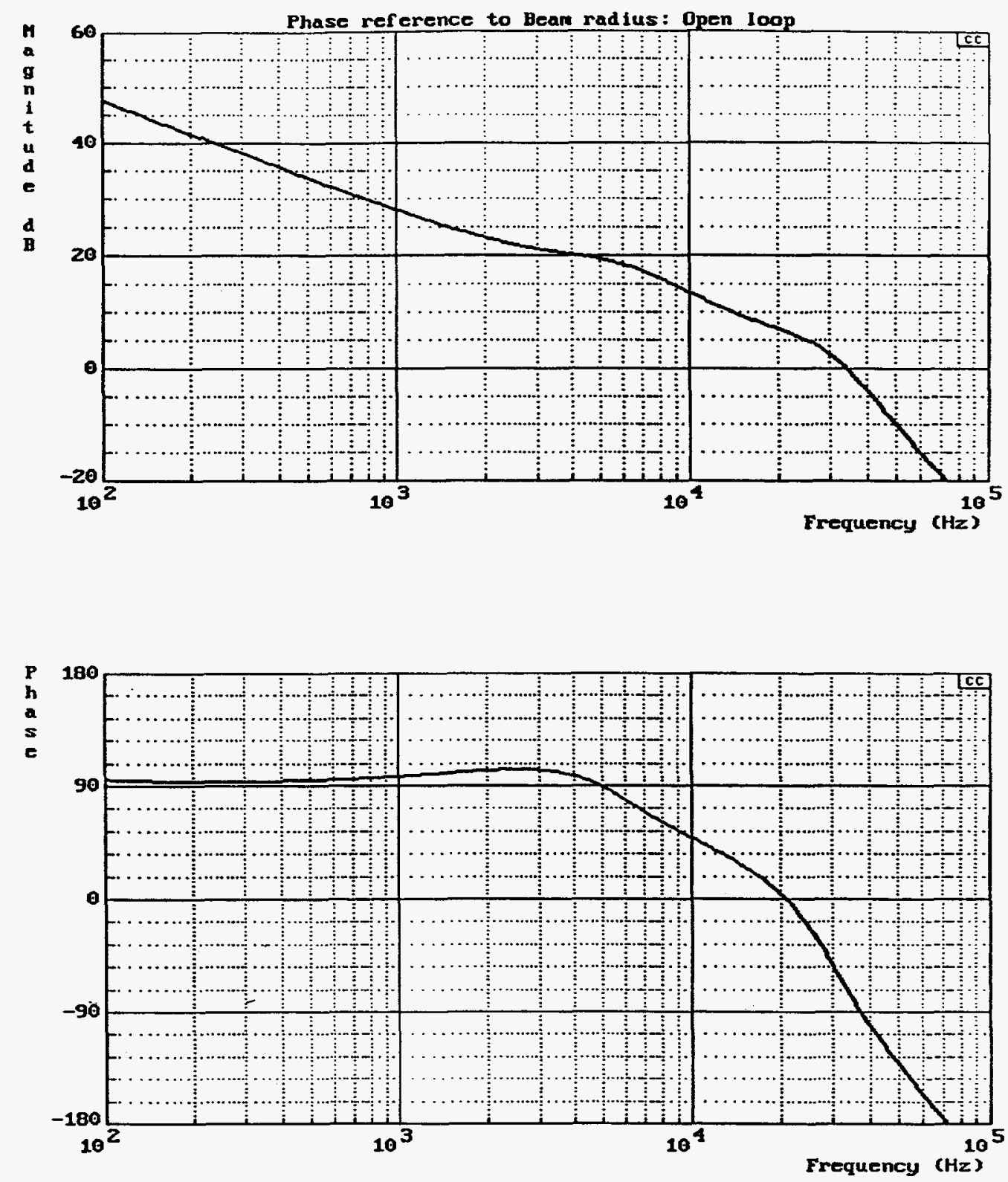

Figure 4.11. A calculation of the open loop response of the radial -loop with the closed loop phase loop embedded. The raising gain at low frequencies is important for good performance of the radial loop. 


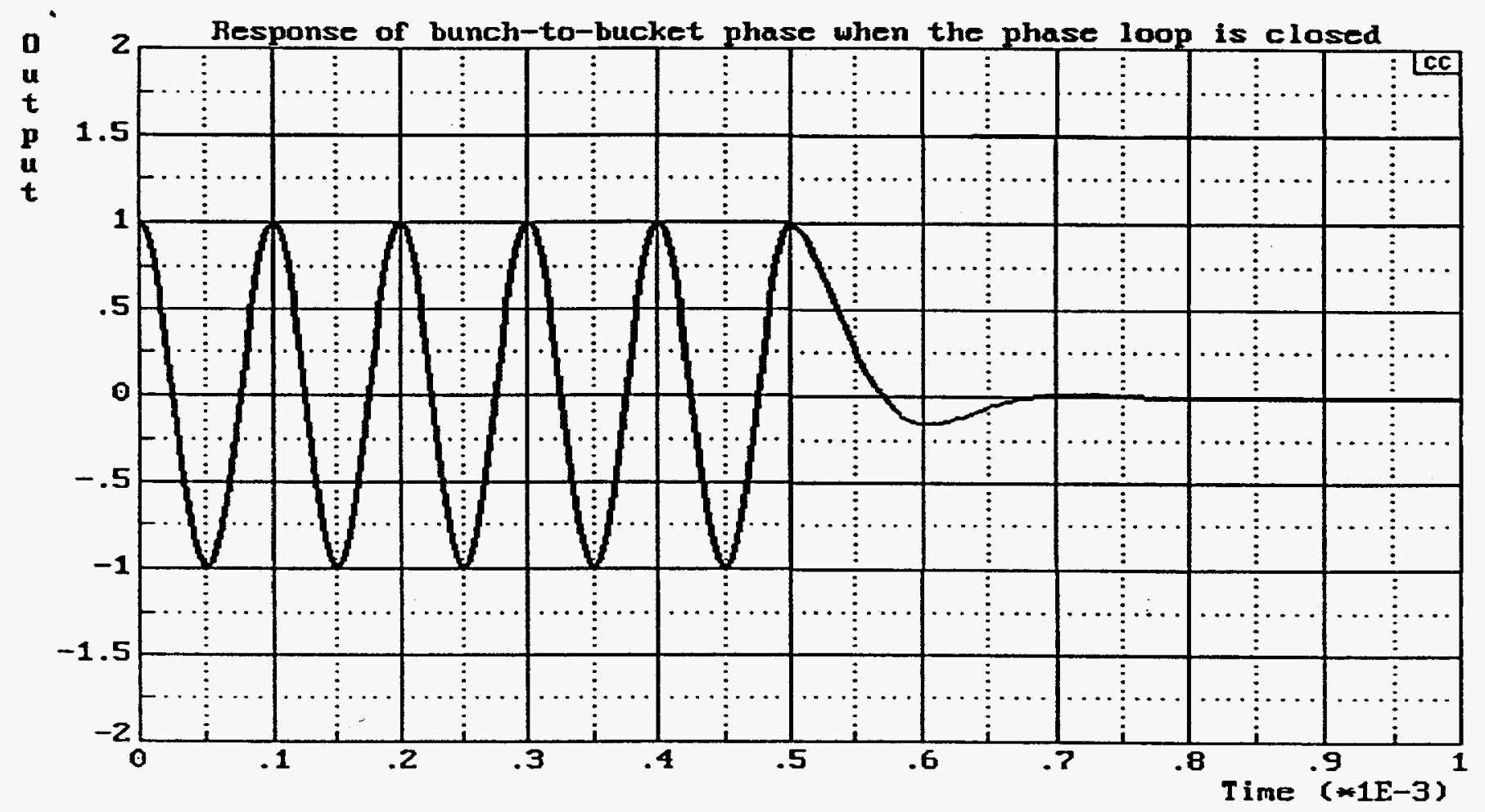

Figure 4.12. A calculation of the transient response of the phase loop, damping an injection phase error. 


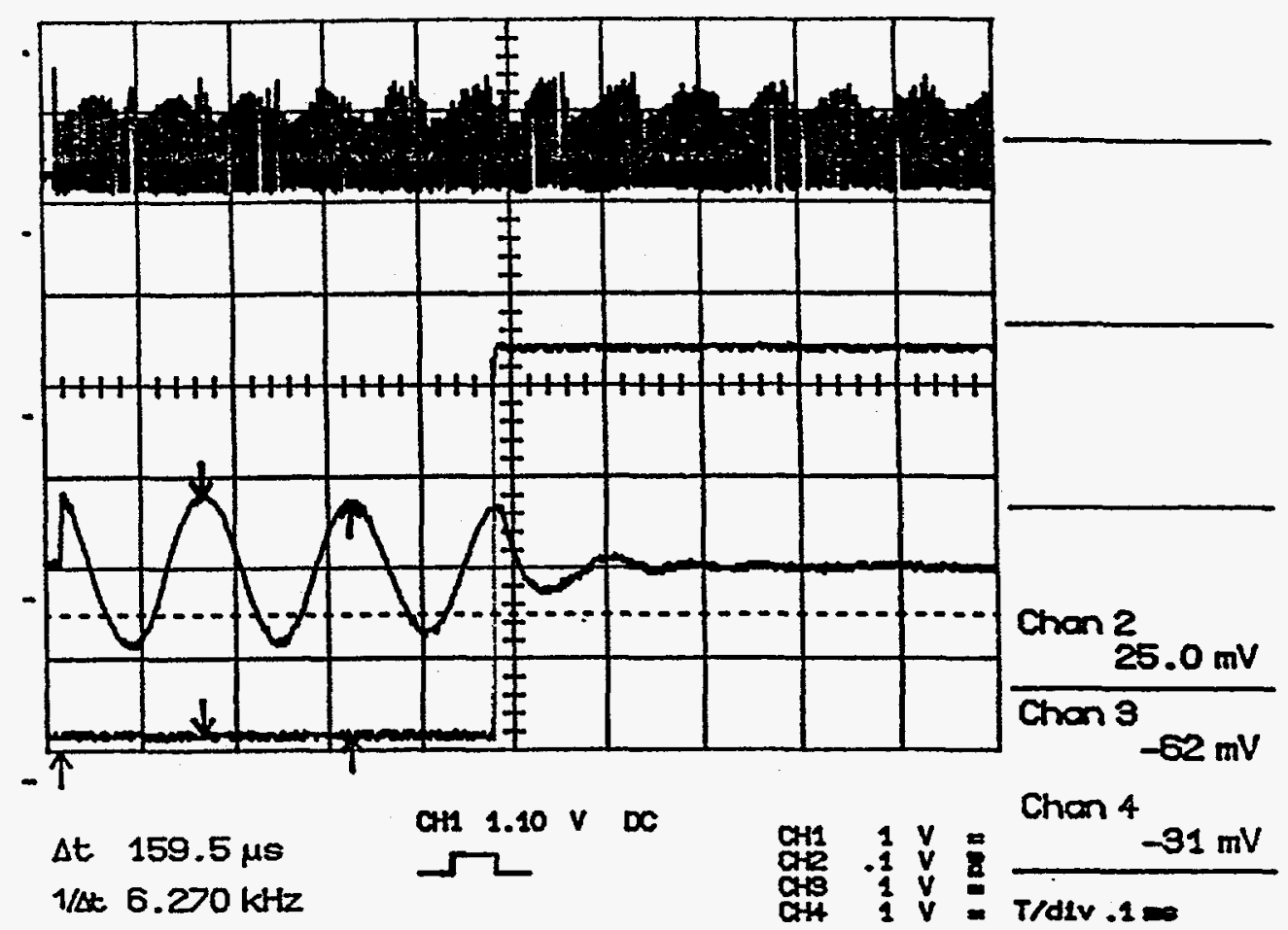

Figure 4.13. A measurement of the transient response of the phase loop. An injection error is created by mis-adjusting the phase of the chopped proton beam. Top trace is the longitudinal pick-up, wall current monitor. Trace with the step indicates closing of the phase loop. Bottom trace is the phase. 


\subsection{Radial Loop}

4.2.1 Principle of Operation. In Figure 1.1 one sees that the beam position is measured with a transverse pick-up and compared to the reference function, $R(t)$ (see Section 1.3 for details). The difference between the measured beam position and the reference function is amplified in the radial loop amplifier and applied to the $\varphi_{\mathrm{r}}$ phase shifter. A change in $\varphi_{\mathrm{r}}$ will cause $\varphi_{\mathrm{e}}$ to deviate from zero and the phase loop will quickly act to return $\varphi_{\mathrm{e}}$ to zero by changing the cavity phase with respect to the beam, $\varphi_{\mathrm{b}}$. The beam's rate of energy gain (loss) from the rf cavities will change. If the beam's rate of energy gain is now less than the rate of rise of the magnetic field, then the beam will begin to move toward a smaller radius. The phase $\varphi_{\mathrm{r}}$ controls the rate of change of the radius. If the reference function is a constant then eventually the radius will equal the reference function and the phase $\varphi_{\mathrm{r}}$ will become zero, then the system will assume its quiescent state with $\varphi_{\mathrm{b}}$ equal to $\varphi_{\mathrm{s}}$.

The radial loop is called into action in basically four different situations: (1) the beam position is made to follow changes in the reference function; (2) the calculated angle $\varphi_{\mathrm{s}}$ is not exact and $\varphi_{\mathrm{r}}$ is made equal to the negative of the error in $\varphi_{s}$; (3) the apparent value of $\varphi_{\mathrm{b}}$ is not equal to the actual value because of different phase shifts in the cables that bring the cavity voltage and beam longitudinal pick-up signal to the phase detector, $\Delta \varphi$ (this frequently happens if the cable lengths are not exactly matched and the rf frequency changes); (4) any finite dc offset in the phase detector or phase loop amplifier causes $\varphi_{\mathrm{e}}$ to be not exactly zero. Irrespective of number 1 , numbers 2,3 , and 4 imply that the radial loop must always be functioning in order that beam control system operate stably.

One might observe that the radial loop would function as well if the output of the radial loop amplifier was subtracted from the output of the phase detector before going to the phase loop amplifier. The essential difference is that the phase error, $\varphi_{e}$, would then not be always zero on the time scale of the radial loop. It is a technical point and somewhat a matter of taste as to whether the phase shift caused by the radial loop is introduced in a phase shifter (as it is here) or in a phase detector.

Because the phase detector is at the heart of the phase loop and should be as fast as possible, the choice was made to keep it as simple as possible and not to optimize for a linear transfer curve (it is a double balanced mixer operating at 10.7 MHz, see Section 3.2.1) On the other hand, one can readily make a phase shifter with a control bandwidth much faster than the radial loop and a linear . range greater than $\pm 2 \pi$. This means that the control signal into the $\varphi_{\mathrm{r}}$ phase shifter is an absolutely calibrated signal $\left(50^{\circ} / \mathrm{Volt}\right)$ and is very useful for detecting and correcting errors 2 to 4 listed above. 
4.2.2 Dynamics of the Radial Loop. High gain is desirable in the radial loop so that the beam radius closely follows the reference function. However, high gain in the radial loop generally reduces the stability of the system. The fact that the radial loop gain cannot be made arbitrarily large is the main reason for implementing the feedforward system for the stable phase angle, $\varphi_{\mathrm{s}}$. If the radial loop had to provide all the phase shift for $\varphi_{s}$, then the beam position would differ from the reference function by $\varphi_{s} / \mathrm{k}(\mathrm{k}=$ the radial loop gain), which we shall soon see may not be small.

Seen from the perspective of the radial loop depicted in Figure 1.2, the phase loop is just another block in the forward signal path. This block has, however, rather complicated internal dynamics, as described above. We examine now the behavior of the system of the combined radial and phase feedback.

In the first case consider the simplified phase loop, where we ignore the cavity response, the delays, and the PID loop compensator. Equation 4.3 gives the transfer function of the phase loop as it would appear to the radial loop. From Figure 1.2, the complete open loop transfer function for the radial loop is

$$
G_{r}(s)=D_{r}(s)\left(\frac{\delta \omega_{r f}}{\varphi_{r}}\right) B_{R}(s)
$$

Using the beam transfer function for rf frequency to radius from Equation 1.4, one gets for the closed loop transfer function for the radial loop

$$
\frac{\delta R}{R}=\frac{D_{r} g b}{s^{2}+g s+\left(D_{r} g b+\omega_{s}^{2}\right)} .
$$

Comparing Equation 4.8 to 4.3 , we see that the two equations have basically the same form, but the constant term in equation 4.8 has been increased by $\left(D_{r} g b\right)$. The coefficient of the linear term in s remains the same. Recall that the linear term provides the damping. The constant term affects the frequency of oscillation, for under damped motion. In a general sense, the radial feedback has de-stabilized the system by increasing its natural frequency and without increasing its damping.

One can see this more formally by re-writing the denominator of Equation 4.8 ,

$$
s^{2}+g s+\left(D_{r} g b+\omega_{s}^{2}\right)=(s+g / 2)^{2}+\omega_{s}^{2}-(g / 2)^{2}+D_{r} g b
$$

In this form one can read directly the real and imaginary parts of the complex conjugate pair of roots of this expression. The quantity in the squared term with $\mathrm{s}$ is the real part of the roots. The constant term gives the imaginary parts. Figure 4.14 illustrates this point. 


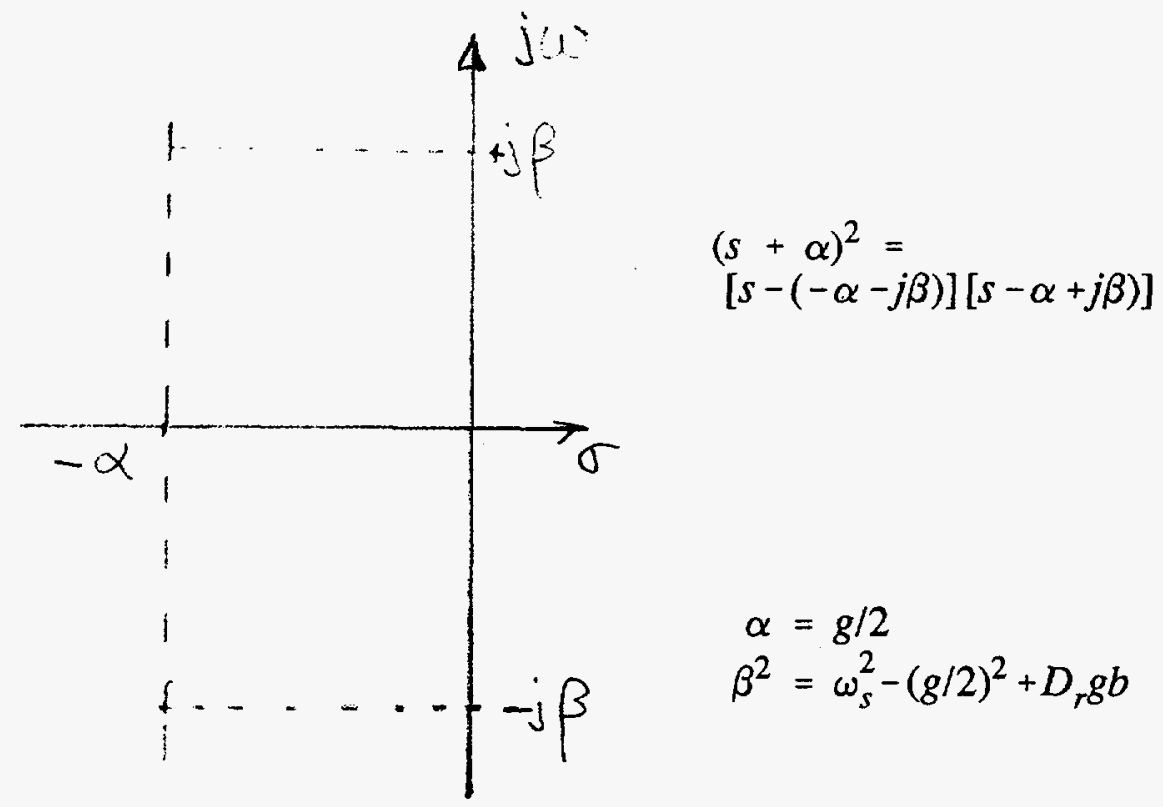

Figure 4.14

For the phase loop we chose a large value of the gain, g, so that the imaginary part of the roots would be zero, to get no oscillation. The new term from the radial loop adds to the value of the imaginary part, it counteracts the damping and can cause an oscillation frequency even higher than the synchrotron oscillation frequency. For this reason, we must always make a compromise between high gain in the radial loop and stability.

The conclusions are qualitatively the same when the full complexity of the phase loop is included in the analysis. One important difference that does arise in a realistic analysis is that in addition to limitations on the gain, some compromise must also be made in the bandwidth of the radial loop. A simple compensator is used in the radial loop amplifier with a single-pole roll-off at 1 $\mathrm{kHz}$. A measurement of the radial loop amplifier is shown in Figure 4.15

Combining this with the closed loop transfer function for the phase loop, we show the open loop transfer function of the radial loop in Figure 4.16. The gain increases as $1 / \mathrm{f}$ at low frequency to give good tracking of the reference function. At about $3.5 \mathrm{kHz}$ the gain reaches one, while the phase margin is about $35^{\circ}$. As we expect the system is not highly damped. Figure 4.17 shows a measurement of the loop operating with $1 \mathrm{GeV}$ protons on a magnetic field flattop. 


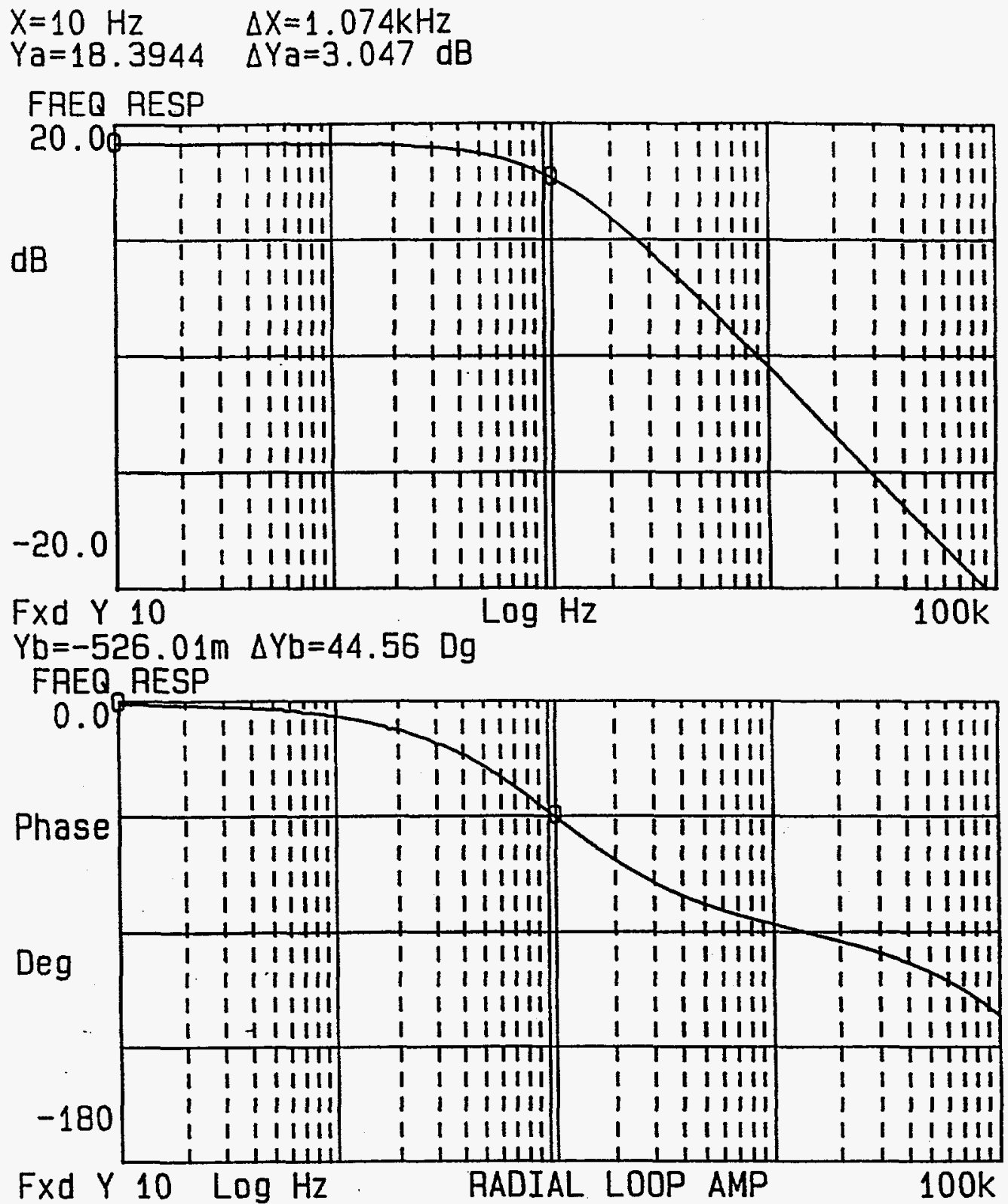

Figure 4.15. A measurement of the frequency response of the radial loop amplifier, showing a single-pole roll off at $1 \mathrm{kHz}$. 



Figure 4.16. A calculation of the open loop transfer function of the radial loop, including the calculation of the closed loop phase loop. 


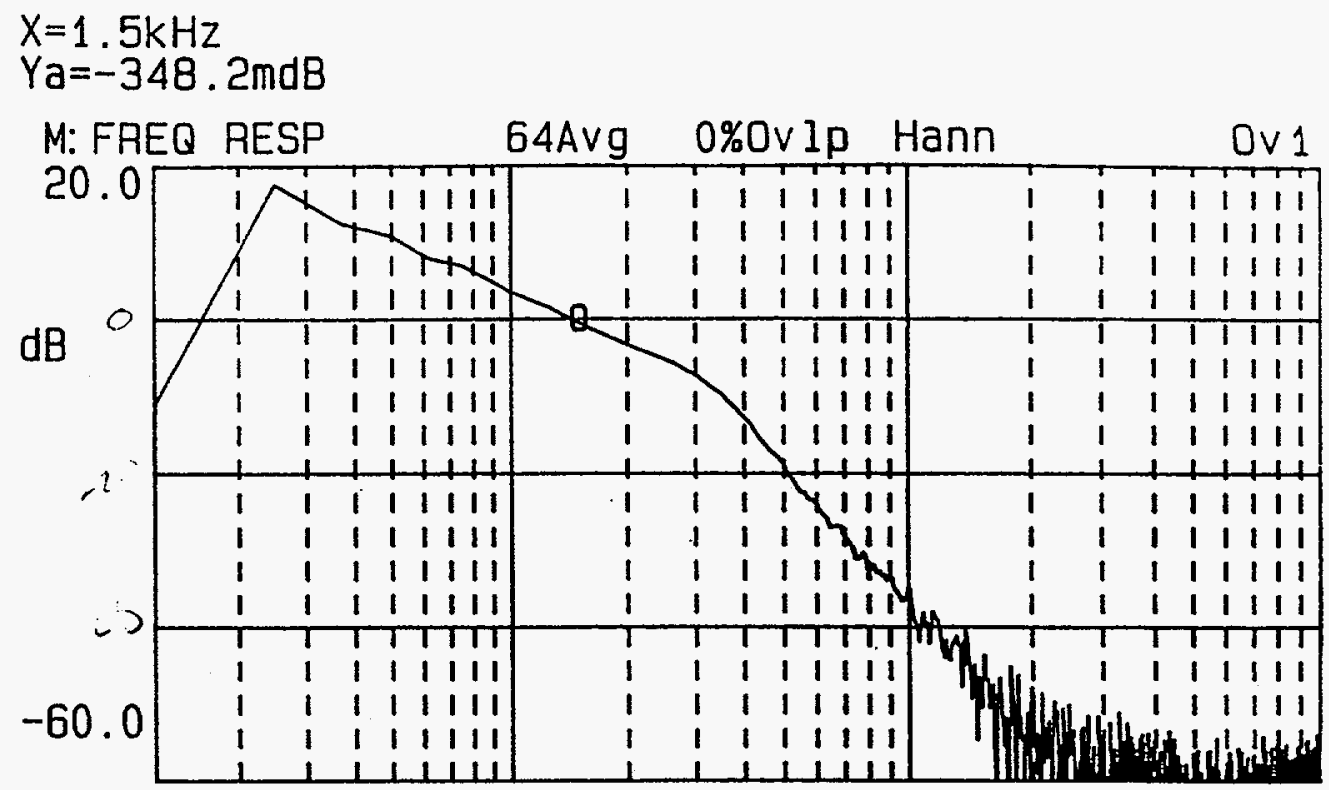

FXd Y $125 \log \mathrm{Hz}$ RADIAL LOOP: OPEN LP $100 \mathrm{~K}$ $Y b=-142.31$ Deg

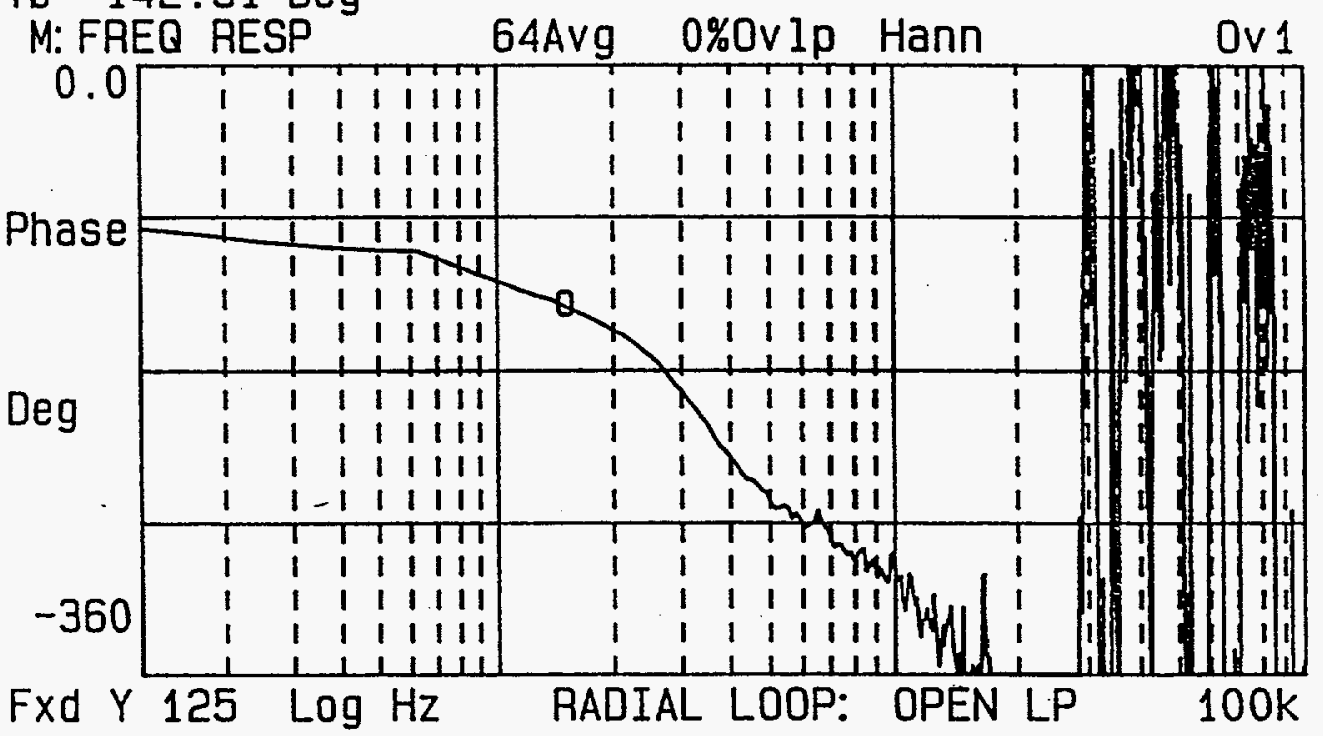

Figure 4.17. A measurement of the radial loop open loop response while operating with beam on a magnetic flattop. 
Figure 4.18 shows the calculated closed loop transfer function, and Figure 4.19 shows the measurement with beam. The peaking in the closed loop gain is characteristic of a system that is optimized for bandwidth (about $6 \mathrm{kHz}$ ) in favor of damping. The calculated step response of the radial loop, shown in Figure 4.20 at two values of the synchrotron frequency, indicates the extent of the ringing due to under-damping. Ultimately, the effect on the beam depends on excursions of the bunch-to-bucket phase as a consequence of the radial step. Excessive oscillations here will have the detrimental effects of longitudinal emittance dilution or even particle loss. Figure 4.21 is a calculation of the bunch-to-bucket phase caused by a radial step. Since the deviation of this phase from zero is proportional to the rate of change of frequency, the area under this curve is proportional to the change in frequency (radius). One can see from the figure that the ringing is damped in about $400 \mu \mathrm{s}$. 

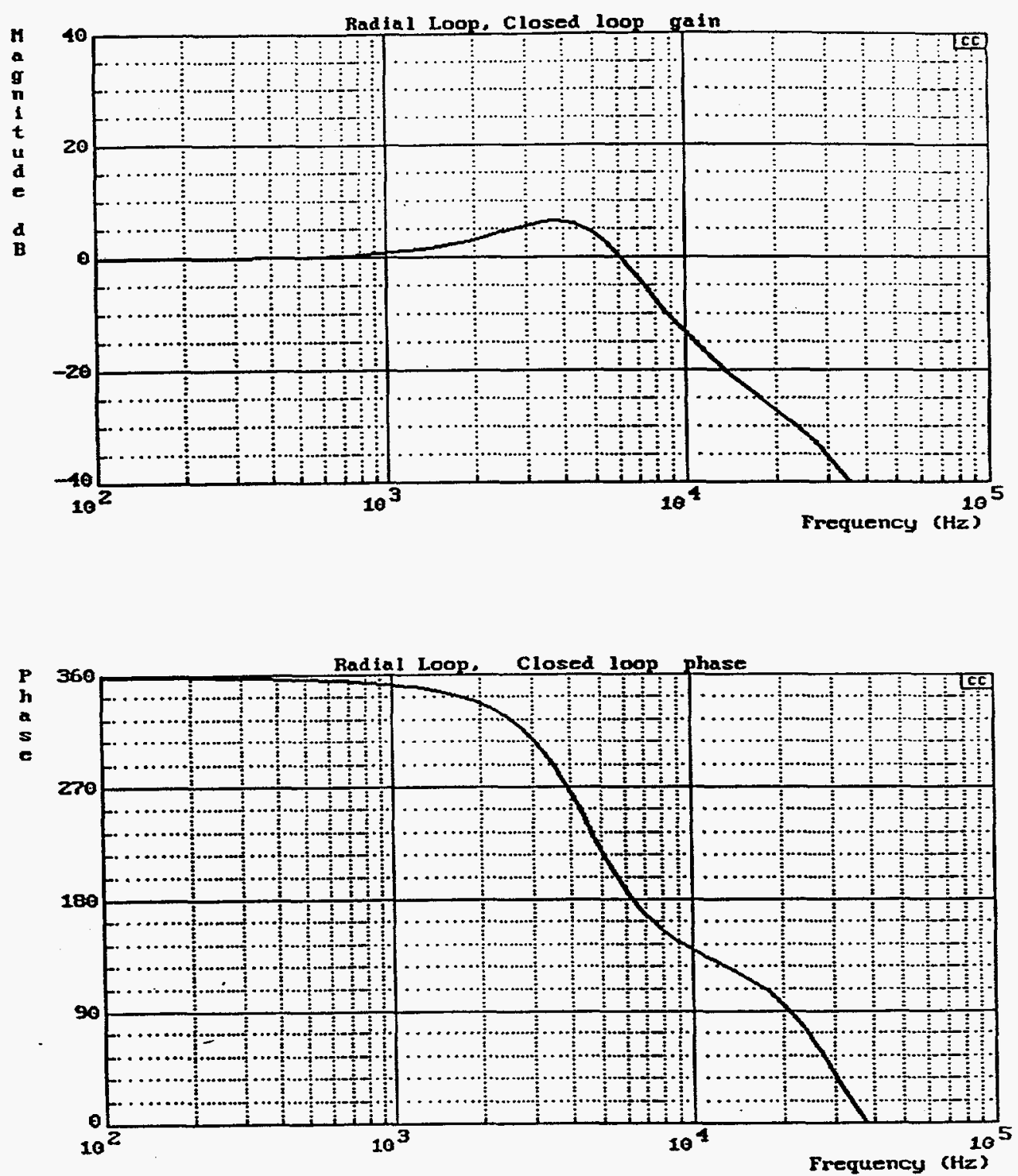

Figure 4.18. A calculation of the closed loop transfer function of the radial loop. 


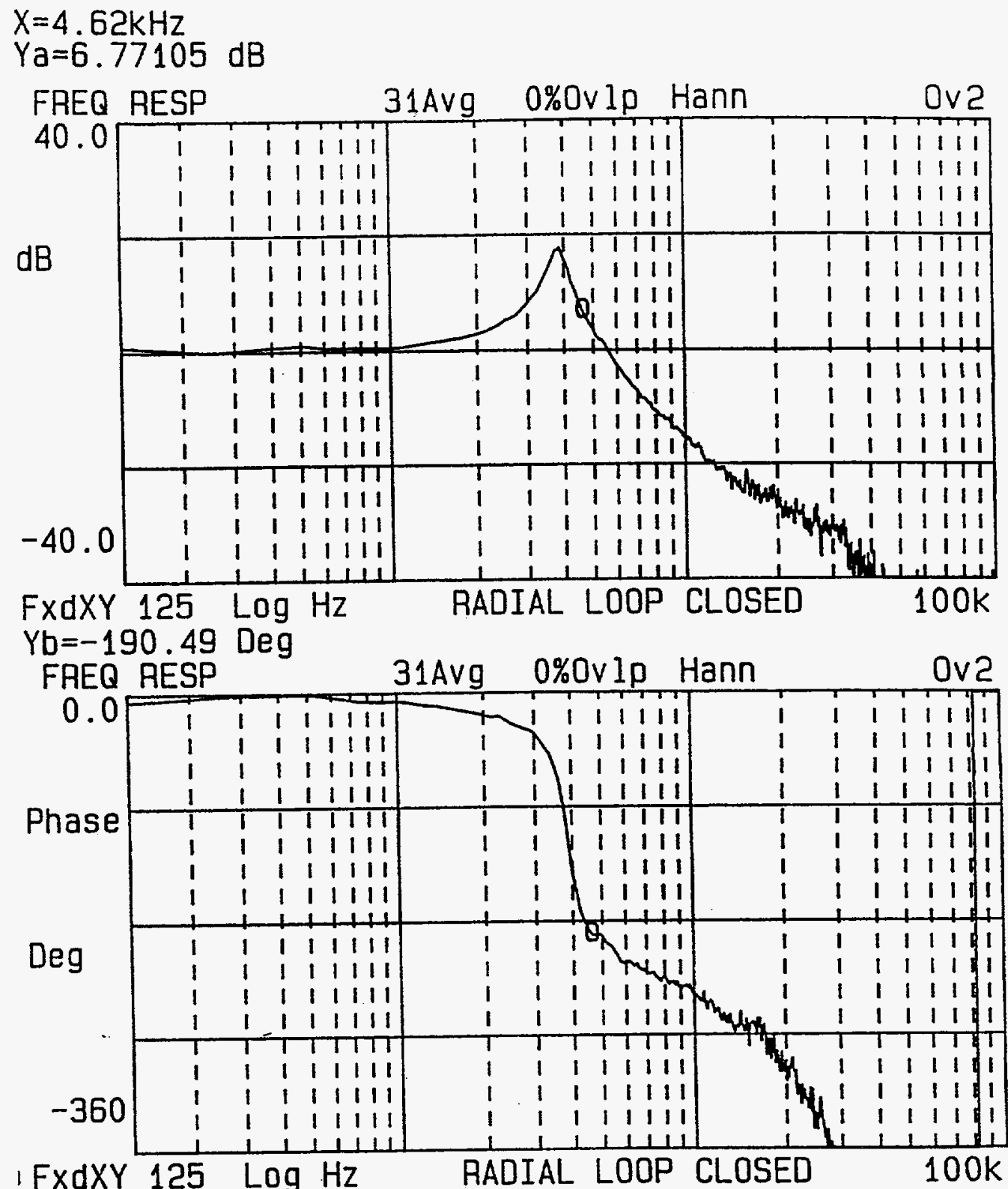

Figure 4.19. A measurement of the closed loop transfer function of the radial loop while operating with beam on a magnetic flattop. 


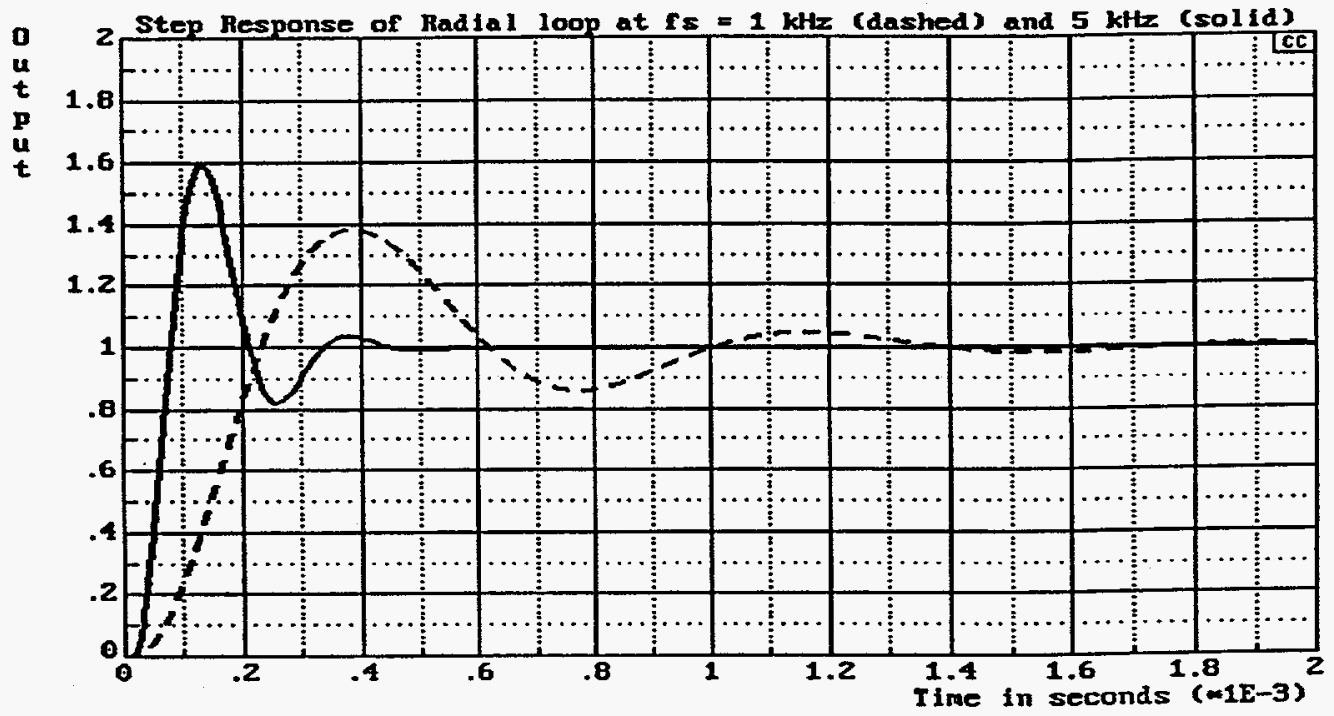

Figure 4.20. A calculation of the step response of the radial loop for two different synchrotron frequencies, $5 \mathrm{kHz}$ (solid line), 1 $\mathrm{kHz}$ (dashed line). 


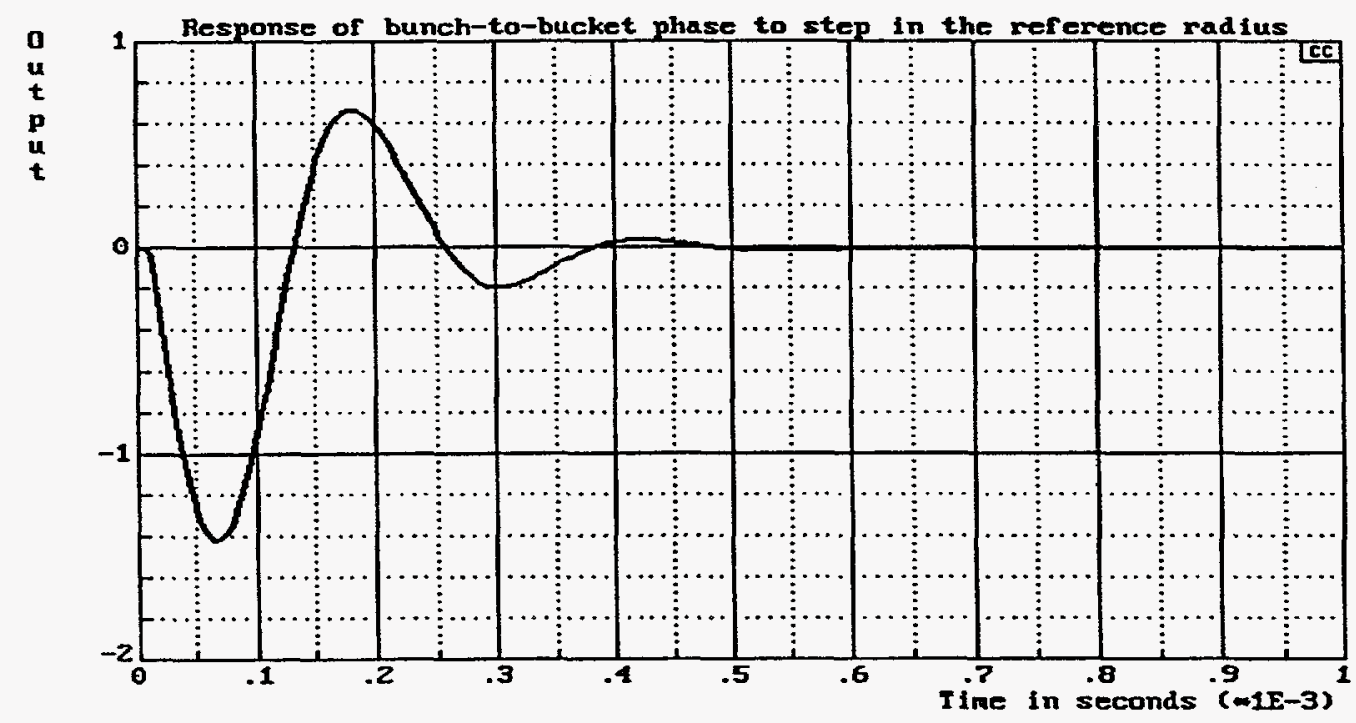

Figure 4.21. A calculation of the bunch-to-bucket phase in response to a step input to the radial loop. 
4.2.3 Low Frequency Errors. An important measure of the performance of the radial loop is the error in the beam position from the reference value. To see how this error depends on the parameters of the system, we use the final value theorem with the closed loop transfer function to find the response, $\delta \mathrm{R}$, to a step function for the reference radius. In the low frequency limit we can neglect the cavity response, the differentiator in the PID controller, the spurious high frequency pole and zero of the phase loop amplifier, and delays. With these approximations the transfer function for the PID controller becomes (see Equation 4.5),

$$
D_{\varphi}(s)=k_{p}^{\prime} \frac{\left(s+1 / \tau_{I}^{\prime}\right)}{\left(s+1 / \tau_{p}\right)}
$$

$$
\begin{aligned}
& \text { where } \bar{\tau}_{\mathrm{p}}^{-1} \text { is the measured low frequency pole of the loop } \\
& \text { amplifier. }
\end{aligned}
$$

The Laplace transform of a step function input on the reference radius is $R(s)=$ $R_{0} / s$. The $1 / s$ here cancels with the $s$ of the final value theorem (see Appendix 7.1),

$$
\underset{t \rightarrow \infty}{\operatorname{limit} f(t)}=\underset{s \rightarrow 0}{\operatorname{limit}} \mathrm{sF}(s)
$$

To find the relative position error, we need the limit of the open loop transfer function as $\mathrm{s}$ goes to zero to evaluate

$$
\frac{R_{o}-\delta R(\infty)}{R_{o}}=1-\frac{G_{r}(s)}{1+G_{r}(s)}=\frac{1}{1+G_{r}(s)}
$$

where $G_{r}(s)$ is the open loop transfer function of the radial loop

$$
G_{r}(s)=k_{\theta} D_{r}(s)\left(\frac{\delta \omega_{r f}}{\phi_{r}}\right) B_{R}(s) k_{p u},
$$

where $\mathrm{k}_{\boldsymbol{\theta}}$ is the phase shifter constant, 50 degrees/Volt, $\mathrm{k}_{\mathrm{pu}}$ is the radius pick-up constant, $1.0 \mathrm{Volt} / \mathrm{cm}$, $D_{r}(s)$ is the transfer function for the radial loop amplifier, $\left(\delta \omega_{\mathrm{rr}} / \varphi_{\mathrm{r}}\right)$ is the closed loop transfer function of the phase loop,

$B_{R}$ is the beam transfer function, see Equation 1.4. 
The transfer function of the radial loop amplifier, $D_{r}(s)$ shown in Figure 4.15, is given by,

$$
\begin{aligned}
& D_{r}(s)+\frac{k_{r}}{\left(s / s_{r}+1\right)}, \\
& \text { where } \mathrm{k}_{\mathrm{r}}=4 \\
& \mathrm{~s}_{\mathrm{r}}=2 \pi \times 1 \mathrm{kHz} .
\end{aligned}
$$

The closed loop transfer function for the phase loop, $\delta \omega_{\mathrm{rf}} / \varphi$, with Equation 4.9 for $\mathrm{D}_{\varphi}(\mathrm{s})$ is given by,

$$
\frac{\delta \omega_{\text {rf }}}{\varphi}=\frac{k_{\varphi} k_{o} k_{p}{ }^{\prime}\left(s+1 / \tau_{I}{ }^{\prime}\right)\left(s^{2}+\omega_{s}^{2}\right)}{\left(s+1 / \tau_{p}\right)\left(s^{2}+\omega_{s}^{2}\right)+k_{\varphi} k_{o} k_{p}^{\prime}\left(s+1 / \tau_{I}{ }^{\prime}\right)(s)} .
$$

Recalling the beam transfer function from Equation 1.4,

$$
B_{R}(s)=\frac{b}{s^{2}+\omega_{s}^{2}}, \quad b=\frac{c e V_{r f} \cos \varphi_{s}}{2 \pi \beta \gamma_{t r}^{2} E}
$$

Combining and taking the limit we find

$$
\begin{aligned}
\operatorname{limit}_{s \rightarrow o} G_{r}(s) & =\frac{k b k_{\theta} k_{p u} k_{\phi} k_{o} k_{p}{ }^{\prime}\left(1 / \tau_{I}{ }^{\prime}\right)}{\omega_{s}^{2} / \tau_{p}} \\
& =4.4\left(\frac{\tau_{p}}{\tau_{I}{ }^{\prime}}\right) \sim 1200
\end{aligned}
$$

where

$$
\begin{aligned}
& k_{r}=4, \mathrm{dc} \text { gain of radial loop Amp, } \\
& b / \omega_{s}^{2}=3 \times 10^{-4} \mathrm{~mm} / \mathrm{s}^{-1} \\
& k_{\theta}=0.87 \mathrm{radian} / \text { Volt, phase shifter constant, } \\
& k_{p u}=0.1 \mathrm{Volt} / \mathrm{mm}, \text { radial pick-up constant, } \\
& k_{\phi}=0.53 \mathrm{Volt} / \mathrm{radian}, \text { phase detector constant, } \\
& k_{o}=2.5 \times 10^{5} \mathrm{~s}^{-1} / \mathrm{Volt}, \text { VCO constant, } \\
& k_{p}^{\prime}=0.32, \text { phase loop PID gain. }
\end{aligned}
$$


Then,

$$
\frac{R_{o}-\delta R(\infty)}{R_{o}}=\frac{1}{1+1200}
$$

As long as the low frequency pole of the phase loop amplifier, $1 / \tau_{p}$, is well below its first zero, $1 / \tau_{I}^{\prime}$, the phase loop appears to the radial loop as a pure integrator, as described in Section 4.1.3, Equation 4.7. The radial loop, then, behaves essentially as a type one servo, with negligible error in response to a step input. The ultimate error is set by the ratio $\tau_{p} / \tau_{I}^{\prime}$ and, in this system, the tracking error given by Equation 4.10 is less than $0.1 \%$.

Unfortunately, other types of errors can occur with significant size. One of practical importance is an error in the stable phase program or in mismatched cables for the beam and cavity pick-up signals. In this case a non-zero value of the phase shift $\varphi_{\mathrm{r}}$ must be maintained, see Figure 1.1. In the formalism that we are using here this implies the beam phase is not zero. This is because the model is based on an expansion of the synchrotron equations about the stable phase angle. If the beam phase is not zero (the model does not know about errors in hardware) then we are considering a case when the beam is being accelerated or decelerated with respect to the synchronous particle, that is, the radius is changing. This then is a velocity type error and one order higher than the error to a step calculated above, and so the final value of the error may not be small.

It is straightforward to find the radius error when phase shift $\varphi_{\mathrm{r}}$ is known,

$$
\varphi_{r}=k_{r} k_{\theta} k_{p u}(R-\delta R) \text {. }
$$

The beam position error is then $0.05 \mathrm{~mm} /$ degree.

It should be noted that the grossest error in the stable phase angle program that is likely to occur happens when the rf harmonic number changes during the acceleration cycle. From Table 1.1 one sees that the electrical phase between the longitudinal beam pick-up and the cavities can change by many degrees, especially when the harmonic number changes from an even to an odd number. For this reason the harmonic number dependence is included in the stable phase angle feedforward system. 


\subsection{Frequency or Phase Control}

4.3.1 Frequency Loop. Sometimes it is desirable to use something other than the radius as the reference for the beam control servo loop. The frequency of the beam or the phase of the beam with respect to reference oscillator, for example, can be used. These references are used in the process of synchronization to the rf cavities in the AGS before bunch-to-bucket transfer between machines. The details of synchronization are presented in Chapter 5.

When the reference for the beam control system is a signal specifying the beam frequency as a function of time we say that the beam is controlled by a frequency loop. Frequency control and radial control are mutually exclusive. At a given magnetic field, the beam momentum can be adjusted by the beam control system but variations in frequency and radius are constrained according to

$$
\gamma^{2} \frac{\delta \omega}{\omega}+\left(\gamma^{2}-\gamma_{t r}^{2}\right) \frac{\delta R}{R}=0 .
$$

It is clear that one cannot specify magnetic field, radius, and frequency independently.

If one then specifies frequency as a function of time, it is necessary that the magnetic field as a function of time is known to insure that the resulting radius is acceptable. Since one of the basic assumptions of the design of the beam control system is that the magnetic field is an independent parameter, not known a priori, then clearly frequency feedback will be used only in special situations.

One special case is during synchronization. Another case is during certain experimental exercises when the beam frequency must be highly stabilized to facilitate measurements of the beam spectra. Also there can be times when very weak beams need to be accelerated that are below the sensitivity of the radius measuring electronics. The phase pick-up electronics has higher sensitivity because it uses the whole signal from the beam, whereas the radius electronics must use the difference in signals from two pick-ups. The frequency can be obtained from the phase pick-up signal. Using frequency loop control to accelerate weak beams requires a very good measurement of the magnetic field as a function of time, and inevitably some trial and error.

The dynamics of a frequency loop are same as a radial loop as long as the frequency measurement is at least as fast as the radius measurement. The beam transfer functions relating beam frequency to rf frequency and beam radius to rf frequency are proportional, see Equations 1.3 and 1.5. The proportionality constant depends on the $\mathrm{rf}$ frequency, $\omega_{\mathrm{rf}}$, the machine radius, $\mathrm{R}_{\mathrm{o}}$, and the frequency slip parameter, $\eta=\left(\gamma^{-2}-\gamma_{\text {tr }}^{-2}\right)$.

$$
B_{R}=\left(\frac{R_{o}}{\omega_{r f} \gamma_{t r}^{2} \eta}\right) B_{\omega} .
$$


The only adjustment that is required in changing from radius control to frequency control is to properly scale the dc value of the loop gain.

Figure 4.22 shows a block diagram of the beam control system including the equipment needed for frequency and phase control, and switching between from one to the other. The three beam transfer functions, $B_{\varphi}, B_{r}$, and $B_{\omega}$ are shown. The inner feedback loop is the phase loop and is always running. Which one of the three outer loops is operating is controlled by the position of the indicated switches. Each one of the three outer loops has its own reference input, $R(t), \omega_{\text {reference, }} \varphi_{\text {sync }}$.

Feedback for the radial loop is indicated in the block labeled $\mathrm{k}_{\mathrm{pu}}$. It has a sensitivity of 1 volt $/ \mathrm{cm}$. Feedback for frequency is block $\mathrm{k}_{\omega}$. Its sensitivity is $1 \mathrm{volt} / \mathrm{kHz}$. Feedback for the synchronization loop, $\mathrm{k}_{\mathrm{s}} / \mathrm{s}$, is described below.

One sees that the compensators for the three loops, $D_{r}, D_{\omega}$, and $D_{s}$ are arranged in cascade. This is done because the compensator for the radial loop is implemented with one of the standard loop amplifiers of the beam control system and is always operating in the loop. While the compensators for the other loops are simpler, special purpose circuits.

The compensator for the frequency loop is just a frequency-independent attenuator. Its value can be found from

$$
\begin{aligned}
& D_{\omega}=\left(\frac{k_{p u}}{k \omega}\right)\left(\frac{R_{o}}{\omega_{r f} \gamma_{t r}^{2} \eta}\right) \\
& =-20 \mathrm{~dB}, \text { for } 200 \mathrm{MeV} \text { protons, } \\
& =-10 \mathrm{~dB}, \text { for } 1.5 \mathrm{GeV} \text { protons. }
\end{aligned}
$$

A measurement of the compensator, $D_{\omega}(s)$, for the frequency loop is shown in Figure 4.23.

When the switch between radius and frequency feedback is made and the reference frequency corresponds to a new radius, then the frequency loop will respond with its characteristic step response. This is plotted in Figure 4.24. The bunch-to-bucket phase will experience a much faster transient, because of the phase loop. This is the same as shown in Figure 4.21. 


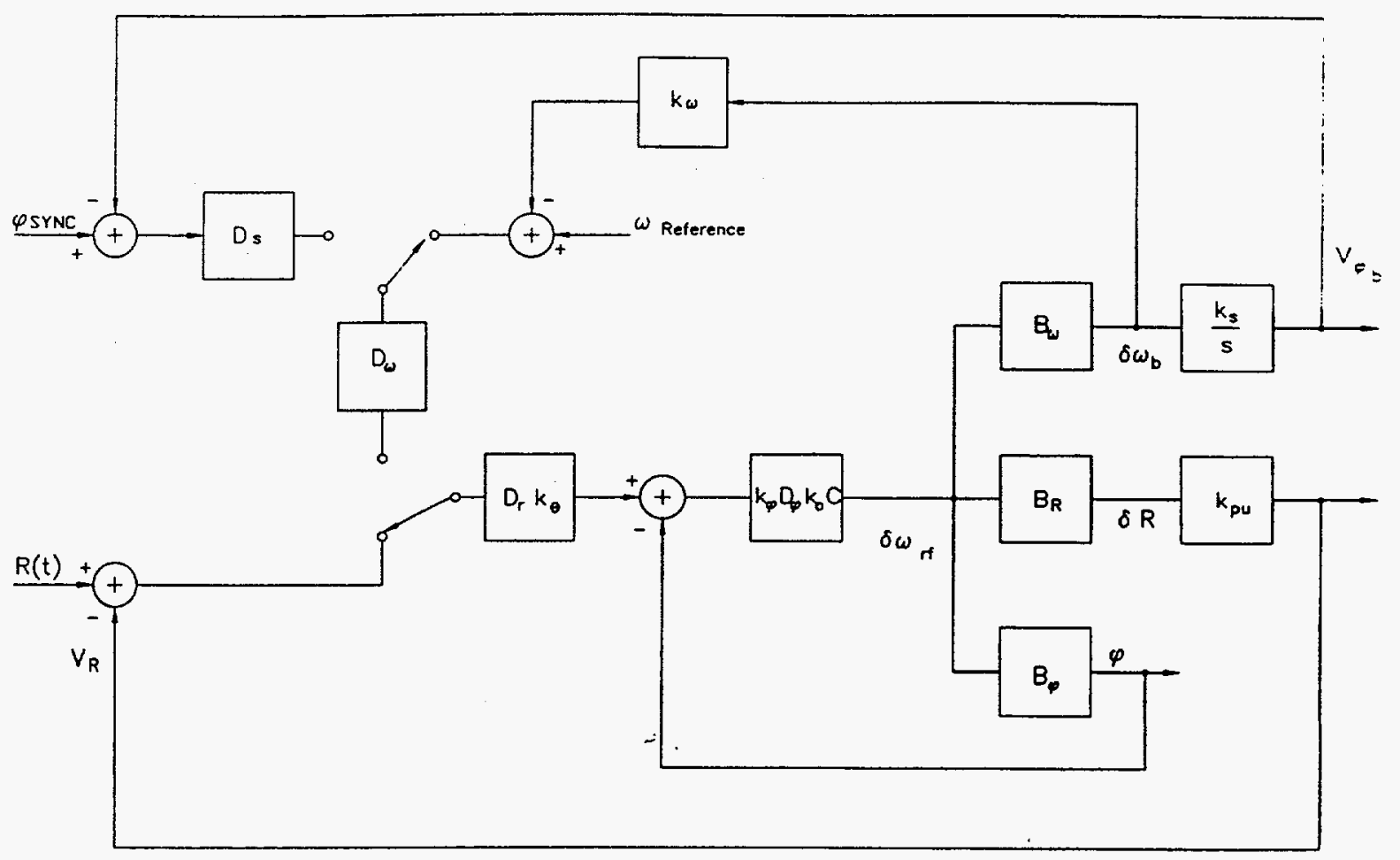

Figure 4.22. Block diagram of the beam control system showing the equipment needed for frequency and phase control. The phase loop is always closed and the switches change from radial to frequency control and from frequency to external phase (synchronization). 


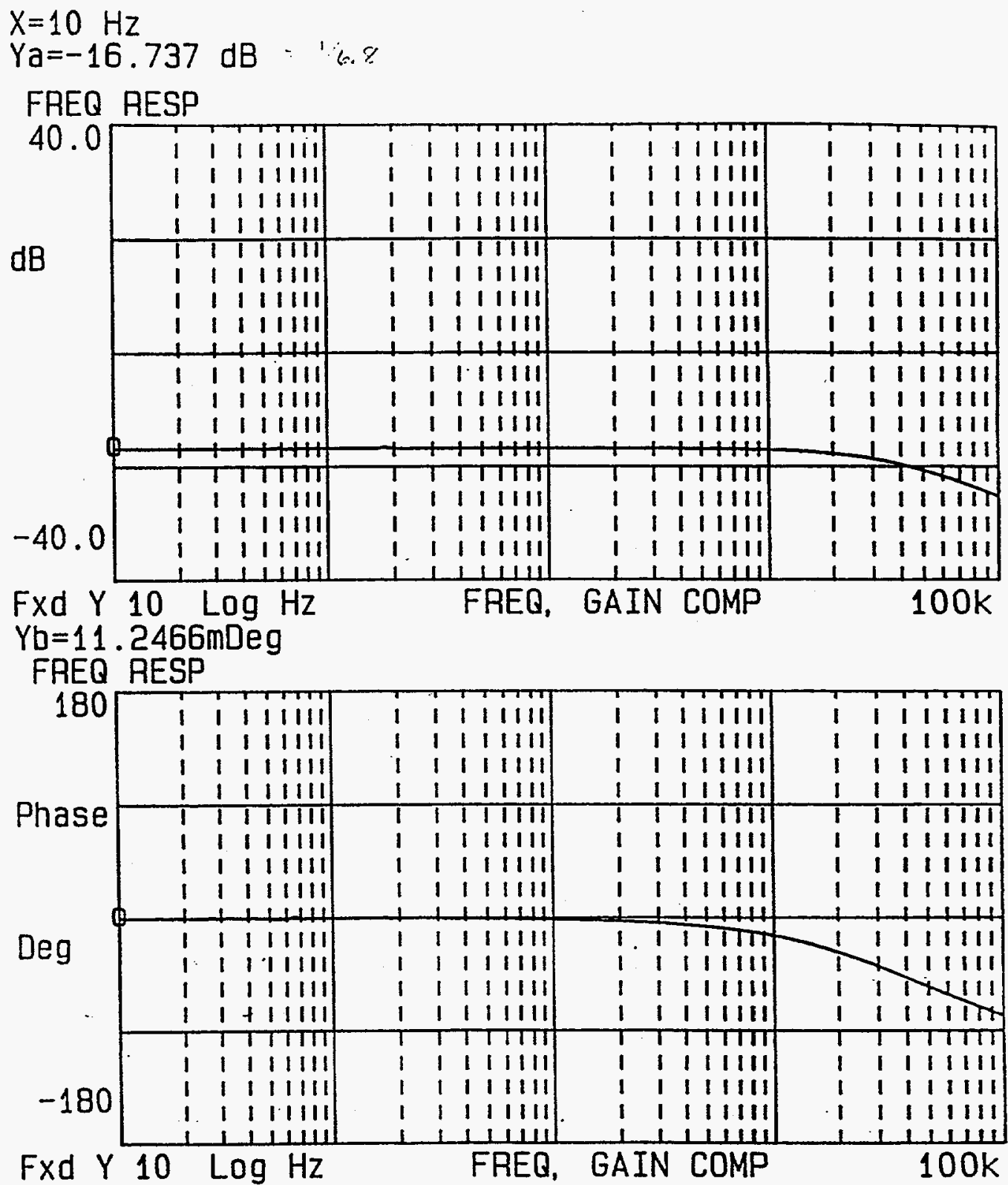

Figure 4.23. A measurement of the frequency loop compensator, ideally it is just a constant. 


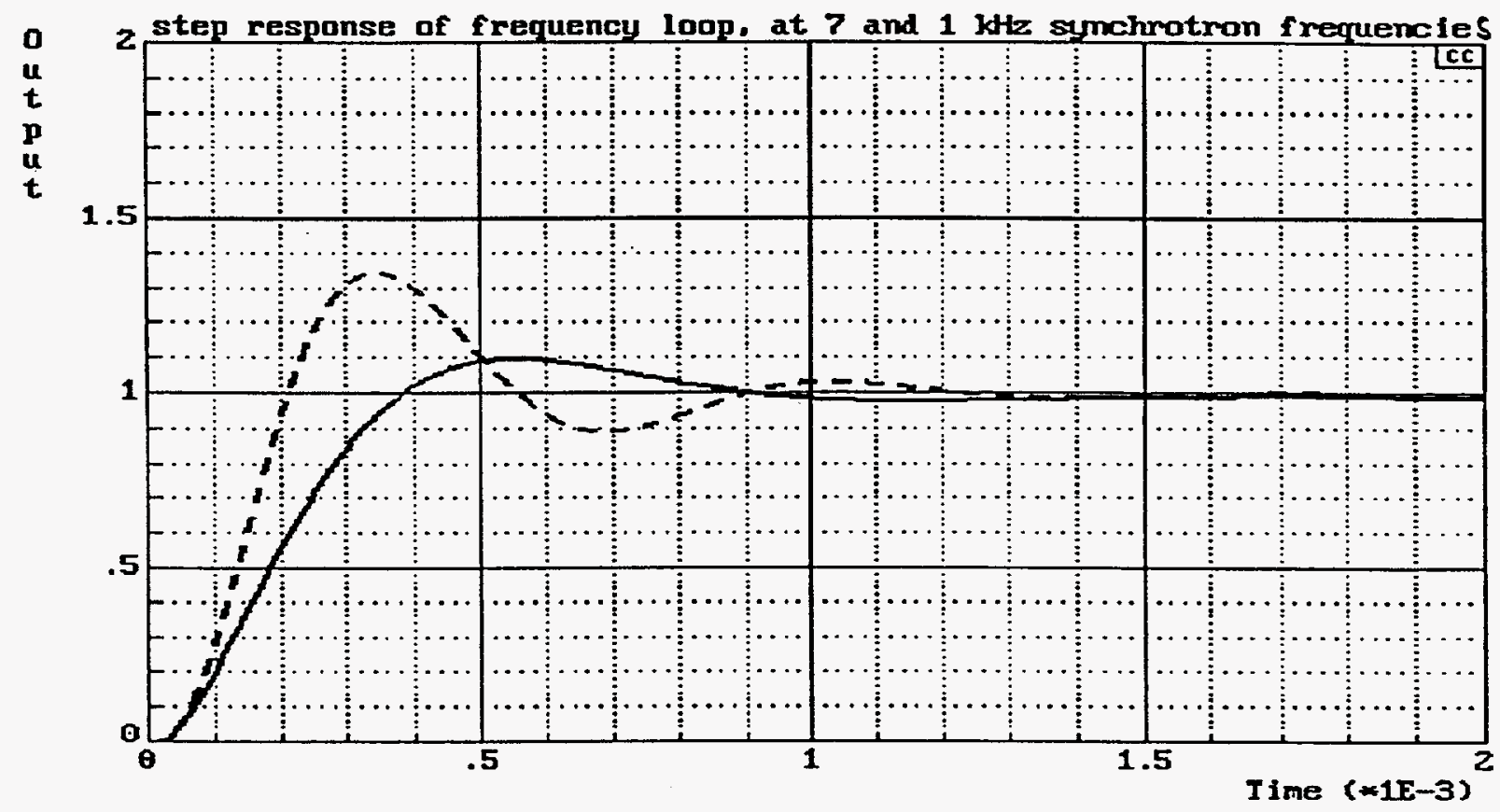

Figure 4.24. A calculation of the step response of the frequency loop for two different synchrotron frequencies, $1 \mathrm{kHz}$ (solid line), $7 \mathrm{kHz}$ (dashed line). 
4.3.2 Synchronization Loop. The term "synchronization loop" refers to the case when the reference radius is replaced with a measurement of the phase of the beam with respect to some external reference oscillator. Because the phase of the beam is held constant with respect to the reference oscillator, it follows that the frequency of the beam must be equal to reference oscillator frequency. The radial position is then controlled by the reference oscillator frequency.

This loop is used in the Booster before the beam is extracted and transferred to the AGS. The vector sum of the cavities' voltage in the AGS is the reference oscillator, so that the bunches will arrive into the AGS buckets at the desired phase, typically zero degrees. The synchronization process is required because of the batch filling of the AGS. Once the first batch has been transferred then the phase of the AGS buckets is no longer arbitrary and the Booster bunches must be adjusted in phase to accommodate the AGS buckets.

The dynamics of the synchronization loop are somewhat different from the radius and frequency loop. This is because the phase measurement process incurs delay. When the beam control system makes an adjustment to the frequency of the beam the change in phase is not instantaneous, the phase accumulates in proportion to the integral of the frequency difference,

$$
\delta \varphi(t)=\int_{0}^{t} \delta \omega_{\text {beam }}(\xi) d \xi .
$$

The Laplace transform of this equation,

$$
\delta \varphi(s)=\frac{1}{s} \delta \omega_{\text {beam }}(s),
$$

and the sensitivity constant of the phase detector, $\mathrm{k}_{\mathrm{s}}(=1.0 \mathrm{Volt} / \mathrm{radian})$, are indicated with the block labeled $\mathrm{k}_{\mathrm{s}} / \mathrm{s}$ in the block diagram of Figure 4.22

The integration inherent in the phase measurement affects the loop stability fundamentally. In fact, the loop would be unstable at any gain if some compensation were not employed. The instability arises because with the phase measurement in the loop we have two integrators in cascade. One is from the phase loop itself, as described above in Sections 4.1.3 and 4.2.3, and the other is from the phase measurement. The total phase shift from the two integrators is $180^{\circ}$ and the system is exactly at the limit of stability. The motion would be completely undamped and with any finite phase shift it would be unstable. 
Formally, this can be seen by considering the open loop transfer function for the synchronization loop. From figure 4.22 we can write the transfer function,

$$
D_{s}(s) D_{\omega} D_{r} k_{\theta} \quad\left(\frac{\delta \omega_{r f}}{\varphi_{r}}\right) B_{\omega} \frac{k_{s}}{s} .
$$

The frequency loop and radial loop compensators, $D_{\omega}, D_{R}$, are essentially constant in the relevant frequency range, i.e., $\omega<\omega_{s}$.

The closed loop phase loop, $\left(\delta \omega_{\mathrm{rf}} / \varphi_{\mathrm{r}}\right)$, behaves basically as a pure integrator, as stated above. This follows from the fact that the phase loop imposes the reference phase, $\varphi_{\mathrm{r}}$, on the bunch-to-bucket phase, see Figures 4.9 and 4.10. A deviation of $\varphi$ from zero will cause an energy gain per turn not matched to $\mathrm{dB} / \mathrm{dt}$, and the beam frequency will deviate from the ideal frequency according to (see Equation 1.1),

$$
\delta \omega_{b}(t)=\omega_{s}^{2} \int_{0}^{t} \varphi_{r}(\xi) d \xi
$$

This integration and the integration from the phase measurement, $\mathrm{k}_{\mathrm{s}} / \mathrm{s}$, appear in cascade and will lead to an unstable closed loop unless $D_{s}(s)$, the synchro loop compensator, is chosen judiciously. It must provide phase lead in the frequency region where the open loop gain crosses one.

For the Booster case,

$$
\begin{gathered}
\left(\frac{\delta \omega_{r f}}{\phi_{r}}\right) B_{\omega} \rightarrow \frac{\omega_{s}^{2}}{s} ; \quad \frac{k_{s}}{s}=\frac{1.0 \text { Volt/radian }}{s} \\
D_{\omega}=1 / 7 ; \quad k_{\theta}=0.87 \text { radian } / \text { Volt } \\
D_{r}(s)=\frac{8.5}{s / s_{r}+1} ; \quad s_{r}=2 \pi \times 1 \mathrm{kHz} .
\end{gathered}
$$


The cross-over frequency is about $2 \mathrm{kHz}$. The compensator shown in Figure 4.25 has a zero at $330 \mathrm{~Hz}$ and poles at $18 \mathrm{kHz}$ and $66 \mathrm{kHz}$. The transfer function for the compensator is

$$
\begin{aligned}
& D_{s}(s)= 0.080 \frac{\left(\tau_{1} s+1\right)}{\left(\tau_{2} s+1\right)\left(\tau_{3} s+1\right)} \\
& \tau_{1}=4.8 \times 10^{-4} \mathrm{~s} \\
& \tau_{2}=9.0 \times 10^{-6} \mathrm{~s}, \\
& \tau_{3}=2.4 \times 10^{-6} \mathrm{~s} .
\end{aligned}
$$

The high frequency pole is due to the finite bandwidth of the amplifiers but does not enter into the analysis.

The effect of the compensator can be seen in Figure 4.26 which is a Nyquist plot of a calculation of the open loop gain. The calculation uses the detailed fits to all the transfer functions of the system. One can see that when the lead-lag compensator is included the critical point, $(-1,0)$, is not encircled by the gain curve, implying stability.

It is often important to know how long it will take to change the phase of the beam with respect to an external reference. The minimum time will be limited by the maximum allowed phase and frequency deviation, and the synchrotron frequency.

For a rough estimate, assume the phase deviation is limited to less than $\pi / 2$, which would be appropriate for a stationary bucket. This is the typical case for synchronization but is not a requirement (see Section 5.3). The beam frequency will follow a linear ramp,

$$
\delta \omega_{b}(t)=\omega_{s}^{2} t
$$

The phase of the beam will increase quadratically with time,

$$
\varphi_{b}-r e f .=\int_{0}^{T} \delta \omega_{b}(\xi) d \xi=\frac{\omega_{s}^{2}}{2} T^{2} .
$$




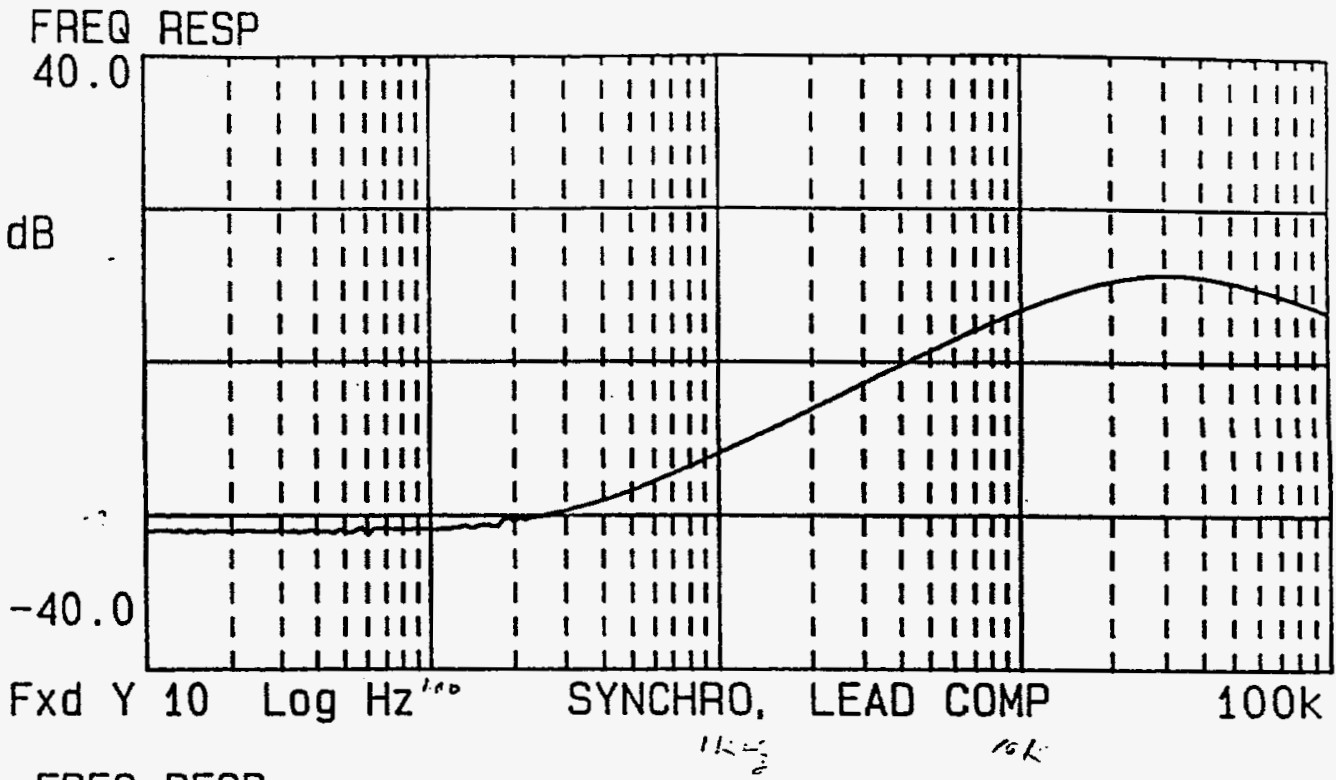

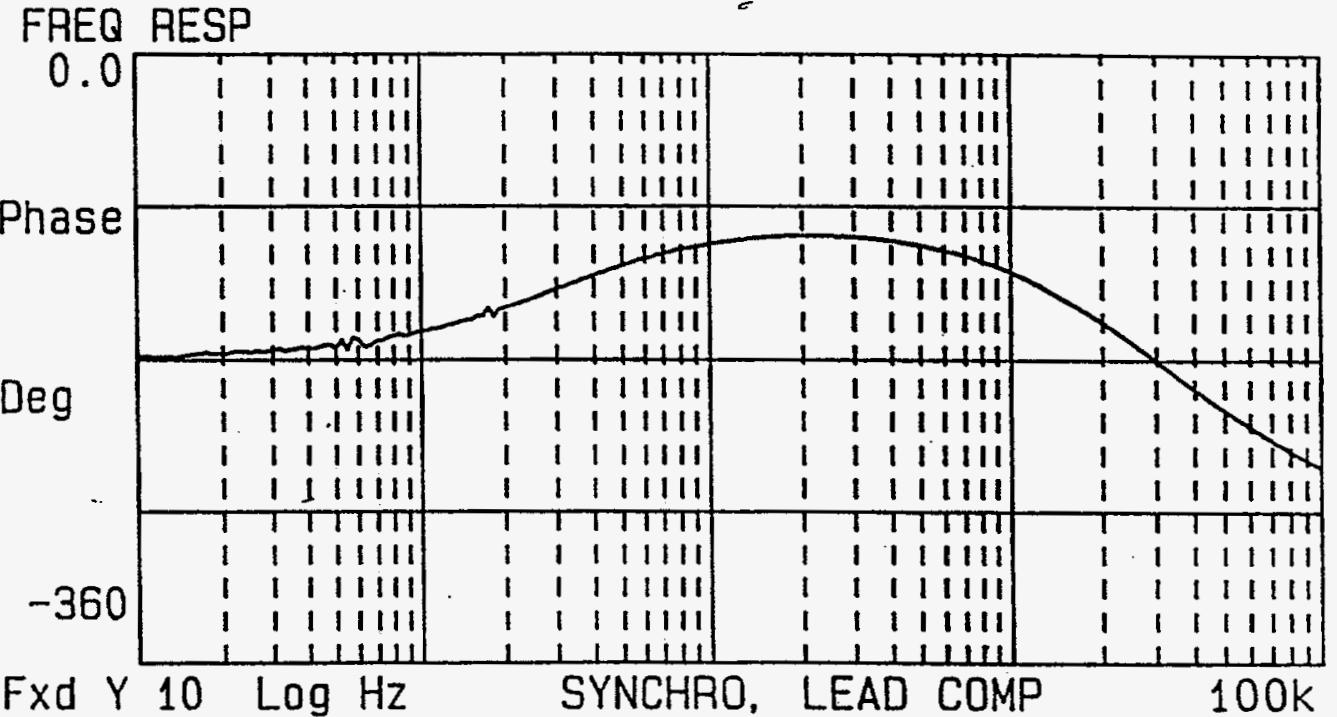

Figure 4.25. A measurement of the compensator for the synchronization loop. See text for fitted parameters. 

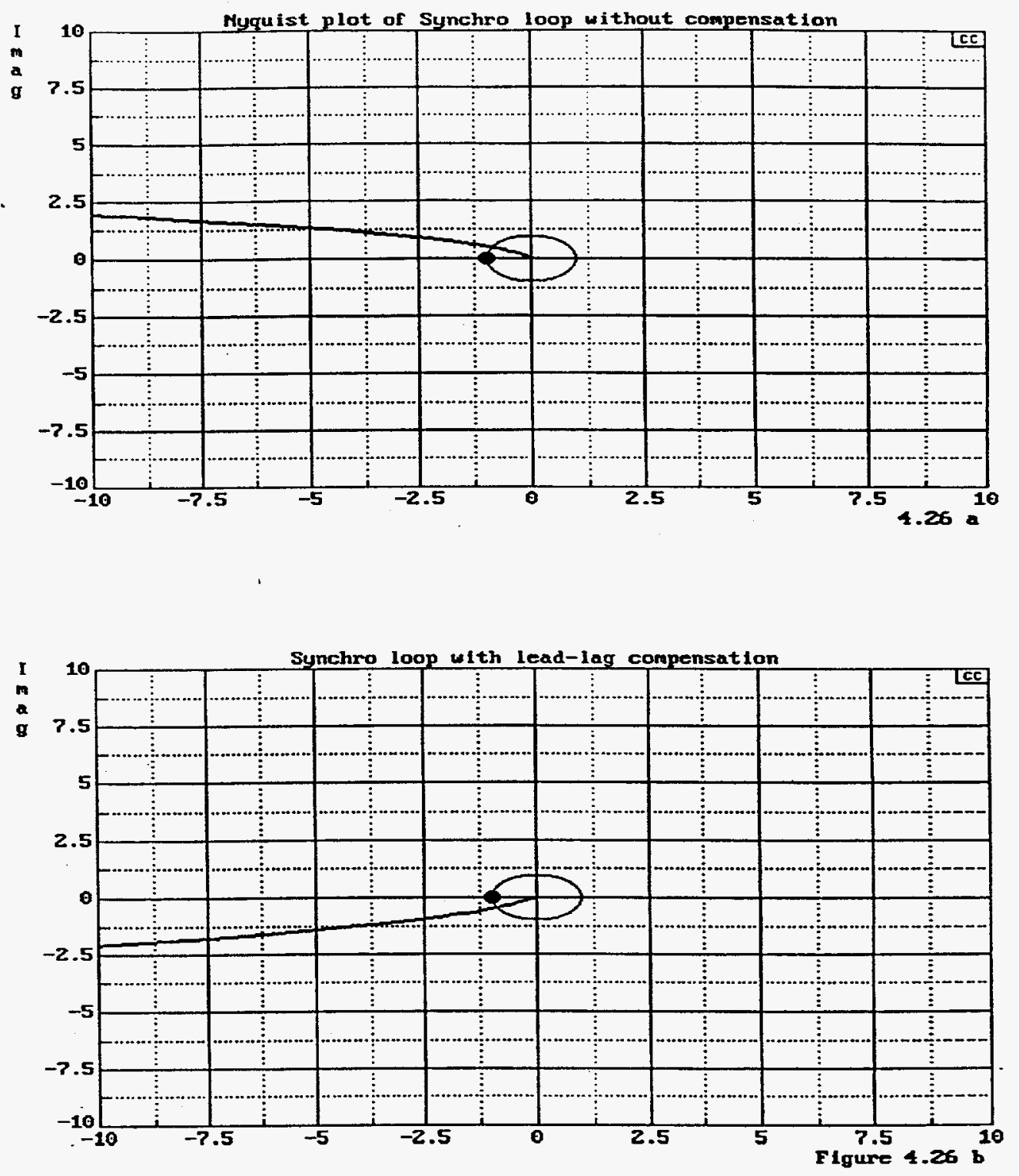

Figure 4.26. Nyquist plots of the synchronization loop. The calculation uses fits to the measured components of the phase and radial loops. Top is without compensation, the critical point is encircled. Bottom is with compensator of figure 4.25 , the point is not encircled. 
If we require a phase change of $\pi$, then the time required is

$$
T=\frac{1}{\sqrt{2} \pi} T_{\text {synchrotron }} .
$$

It should be pointed out that this is not the same operation as shifting the phase of the rf voltage by $\pi / 2$, in an open loop fashion. In the open loop case the beam will carry out a phase oscillation along the separatrix and continually change phase with respect to the rf voltage. The motion along the separatrix is very slow. With the phase loop and synchro loop operating the beam remains at the desired phase of the rf waveform. The rate of change of phase is faster. 


\section{SYNCHRONIZATION AND COGGING}

\subsection{Overview}

Bunch-to-bucket transfer of the beam from the Booster to the AGS requires coupling the If systems of the two machines, so that the bunches will arrive in the AGS buckets at the proper phase. Figure 5.1 shows the arrangement of the coupling between the two machines. The AGS rf system provides a fixed frequency voltage at the cavities. This is done either by deriving the low level drive signal from a fixed-frequency oscillator or by virtue a frequency loop acting on the bunches already present in the AGS.

The figure shows that the reference signal is taken from the vector sum of the cavity voltage pick-up signals. This insures that the effective voltage that the beam will see is used as the reference, as opposed to a drive signal to the cavities, which may differ in phase with respect to the actual voltage due to phase shifts in the power amplifier or miss-tuning of the cavities. A phase shifter in the path of the reference signal adjusts the phase to which the Booster will be locked. This phase is not compensated for variations in phase with changes in frequency or changes in beam time-of-flight between the two machines which changes with beam energy. Since such variations seldom occur, the proper setting of the phase shifter in found empirically by observing the behavior of the bunches in the AGS.

The figure indicates two blocks called "Synchronization" and "Cogging". Synchronization refers to the matching of the rf phases of the Booster bunches to the AGS buckets. Cogging refers to the process of triggering the extraction and injection kickers at the proper times such that the bunches are transferred into pre-selected, empty buckets in the AGS.

The two processes are distinct in function and purpose but do have a common point. That is, they both derive their reference from the AGS rf buckets. A basic difference between them is that synchronization is defined over only one period of the rf frequency, whereas, cogging is defined over the one revolution period of the AGS. They are independent in the sense that the cogging system triggers the injection kicker when the buckets are properly aligned with respect to the kicker, and it is implicitly assumed that the synchronization system has phased the Booster bunches with respect to the AGS rf voltage. It follows then that the bunches must be aligned with respect to the Booster kicker at the same moment that the AGS kicker is triggered. Therefore the two kickers are triggered simultaneously. Fixed delays accommodate the time of flight between the two kickers. 


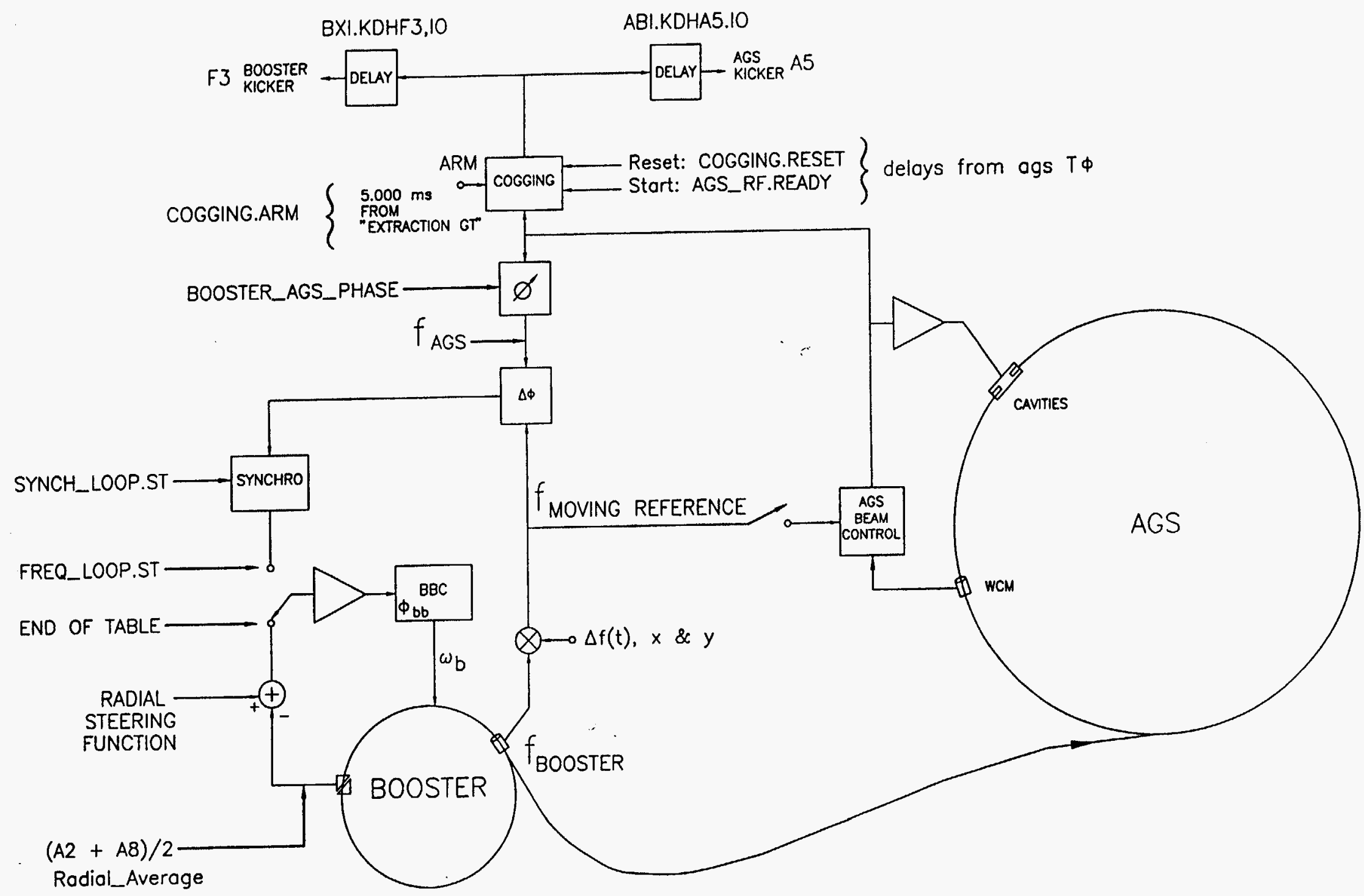

Figure 5.1. The arrangement of the synchronization and cogging equipment. 


\section{$5.2 \quad$ Cogging}

Figure 5.1 shows where the cogging circuit fits within the rf controls systems of the AGS and Booster. Its input is the vector sum signal of the AGS cavity voltages. Its output is trigger pulses to the fast kickers in the AGS and Booster.

The input signal, which is nominally $4 \mathrm{MHz}$, is used as the clock input of a Programmed Logic Device (22V10). The PLD has been programmed to implement two state machines. Figure 5.2 shows the concept of these state machines. One has 12 states, one for each AGS bucket. When the AGS_RF_READY input trigger occurs (when the rf input is known to be stable) the state machine goes to state 1 and advances one state at each rf period thereafter. It thus implements a divide by 12 function with each count (state) identified by a separate variable. States one, four, seven, and ten are marked as lead buckets for the four possible batches of bunch triplets that will be transferred from the Booster to the AGS.

The second state machine has four branches with two states each. AGS_RF_READY puts the state machine into state one. It waits there for an input trigger called ARM. Upon arrival of ARM it goes to state two and waits there for the first state machine to reach state one. When that occurs it outputs a trigger to the kickers. Next it will advance to one of the other three branches. Branch two, for example, which contains states three and four will output a kicker trigger when the first state machine reaches state (bucket) four. Branch three corresponds to bucket seven, branch four to bucket ten.

State machine two does not necessarily go sequentially from branch one to four. From state two it can go to states three, five, or seven. From state four it can go to five, seven, or one, etc. This sequence determines the filling pattern of batches into the AGS. The pattern is controlled by three switches on the front panel of the cogging circuit. Table 5.1 gives the correspondence between switch positions and the filling pattern.

TABLE 5.1

\begin{tabular}{cl} 
Switch Positions & Bucket Triplets \\
\hline 000 & $1 \rightarrow 2 \rightarrow 3 \rightarrow 4$ \\
001 & $1 \rightarrow 2 \rightarrow 4 \rightarrow 3$ \\
010 & $1 \rightarrow 3 \rightarrow 4 \rightarrow 2$ \\
011 & $1 \rightarrow 3 \rightarrow 2 \rightarrow 4$ \\
100 & $1 \rightarrow 4 \rightarrow 3 \rightarrow 2$ \\
101 & $1 \rightarrow 4 \rightarrow 2 \rightarrow 3$
\end{tabular}




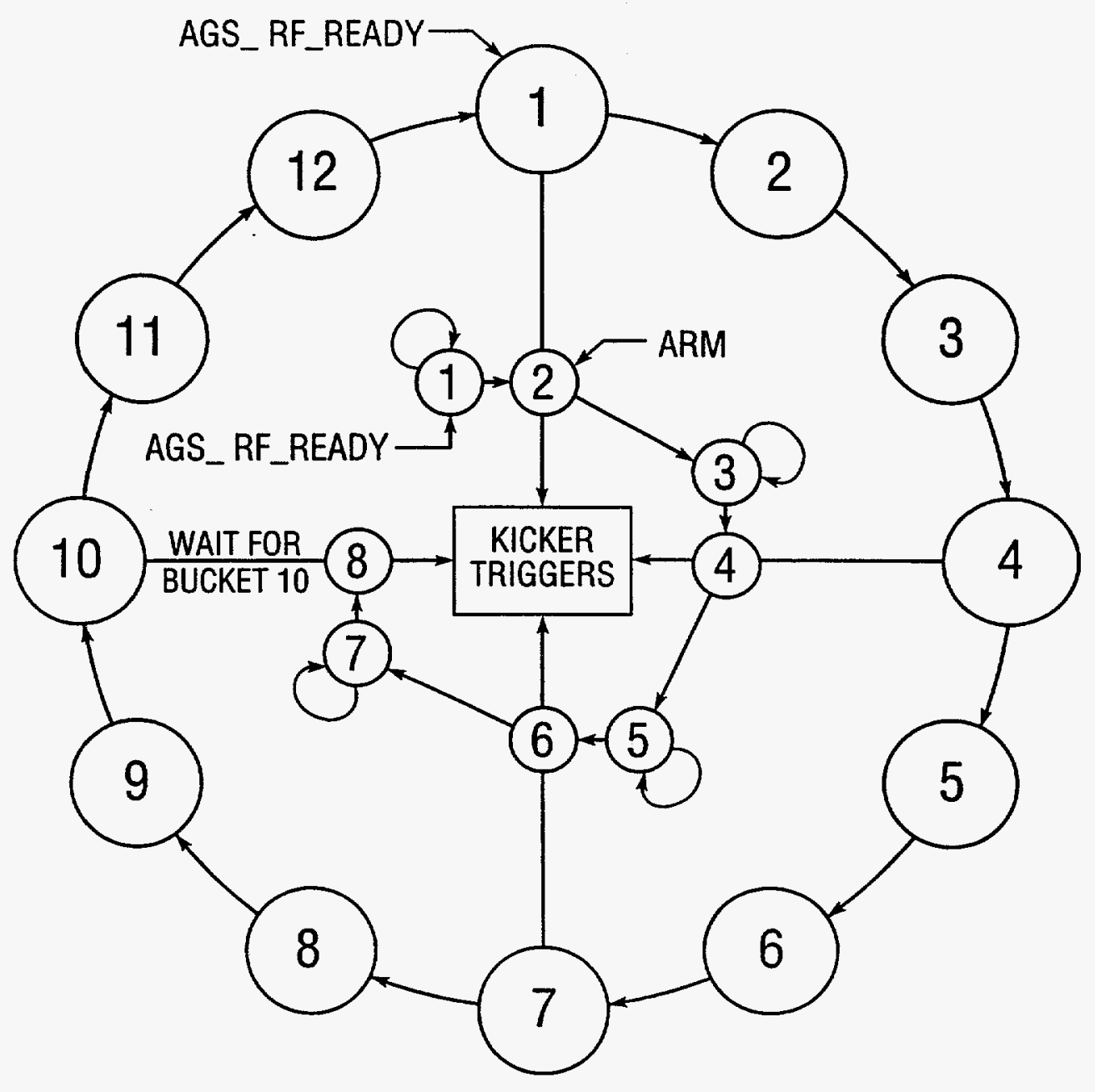

Figure 5.2. State machine diagram of the cogging circuit. The outside state machine counts and labels the AGS buckets. The inside state machine waits in states $1,3,5$, or 7 until the ARM trigger occurs. It then moves to states $2,4,6$, or 8 and waits for the outside state machine to arrive at the appropriate bucket. It then sends out the kicker triggers. 


\subsection{Moving Reference Frame}

The principles of adjusting the phase of the beam to an external reference were described in the previous section on synchronization. A very important practical detail that was not treated in that section is the fact the Booster frequency is not constant at the moment of extraction or before it, when the synchronization operation takes place.

We spoke of measuring the phase of the beam with respect to an external oscillator. Implicit in that discussion is the assumption that the Booster frequency can be made equal to the reference oscillator before the phase adjustment occurs. If the Booster frequency is linearly approaching the reference frequency then the relative phase (modulo $2 \pi$ ) is a sawtooth waveform whose frequency linearly descends through zero. If this phase were used as the feedback signal to the synchronization loop the loop would try to hold the phase constant and equal to the synchro reference value. The result would be that the beam frequency would try to go the AGS frequency as soon as the loop is closed. The resulting radial excursion would drive the beam out of the machine.

This problem is solved by creating a "moving reference frame" in which the synchronization takes place. In the moving reference frame the beam frequency has been translated to a new fixed frequency that is nominally equal to the AGS frequency.

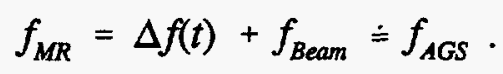

The amount by which the Booster frequency is translated is a time-dependent difference frequency, $\Delta f(t)$, that is zero at the moment of extraction and equal to the difference frequency, AGS - Booster, at the time the synchro loop closes. The phase detector of the synchro loop operates in this moving reference frame where its two input frequencies are nominally equal. Hence, the synchronization process proceeds as if the Booster frequency were equal to the AGS frequency. Figure 5.3 shows a measurement of the Booster beam frequency superimposed with the moving reference frame frequency, which operates for the last $10 \mathrm{~ms}$ of the cycle.

When the synchro loop operates, radial control is relinquished in favor of frequency control. The difference frequency, $\Delta \mathrm{f}(\mathrm{t})$, becomes the determinate of the beam radius. Changes in the magnetic field are not tracked and good reproducibility of the magnetic field is required. In practice this is not a problem. 
TVar: Frequency A

口 Mkr X: $15.5000059 \mathrm{~ms}$ $\mathrm{y}: 3.228486 \mathrm{MHz}$
03 Арг 1992 14: 06: 55 50215 A evts

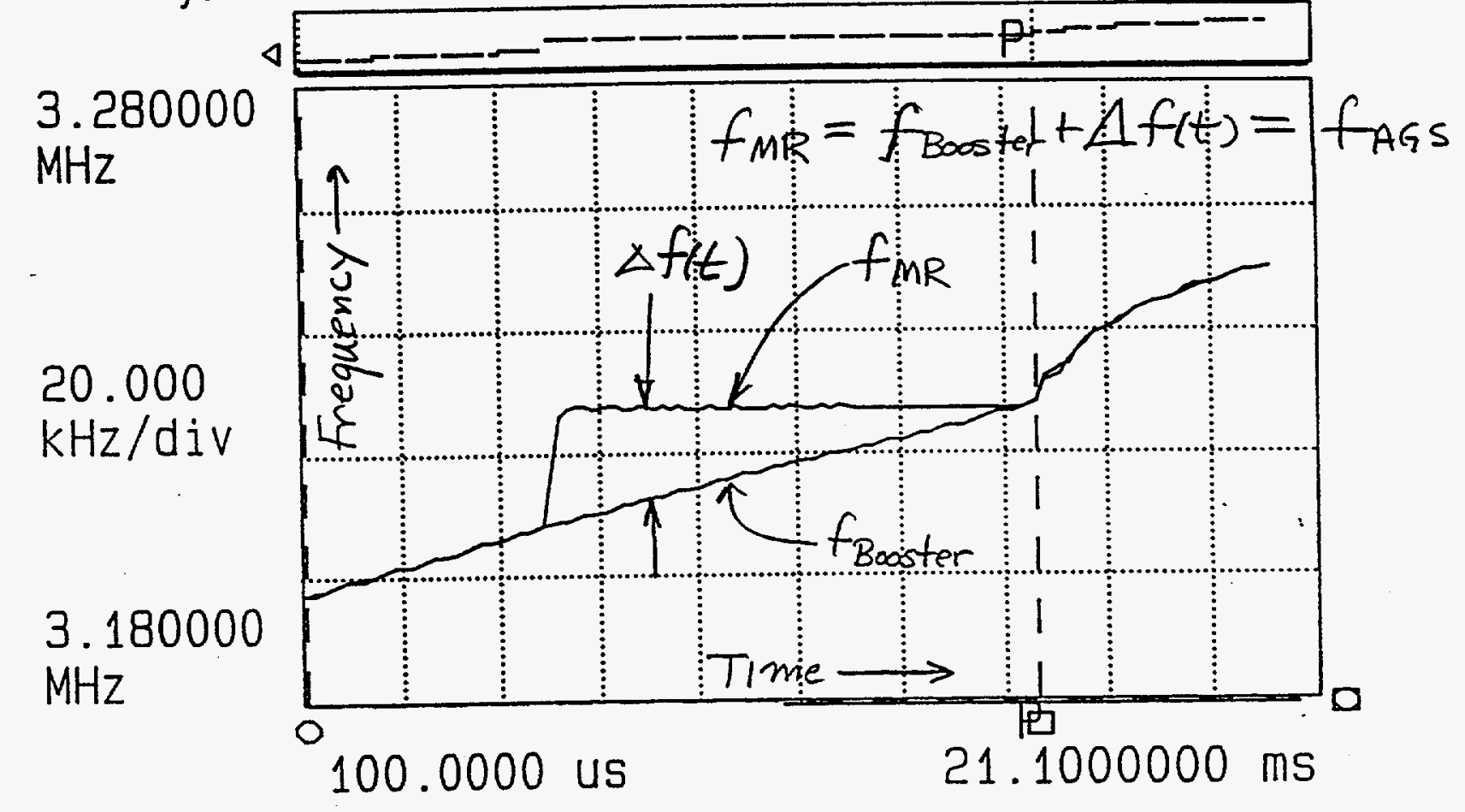

Figure 5.3. Frequency of the Booster during the last $10 \mathrm{~ms}$ of the cycle. Also shown is the Booster frequency translated to the moving reference frame frequency. 
5.3.1 Generation of the Moving Reference Frame. The function, $\Delta \mathrm{f}(\mathrm{t})$, is obtained in practice by measuring the beam frequency when the beam is controlled by the radial loop for the whole cycle. Extraction and transfer to the AGS are suspended for the measurement (the beam is decelerated to injection energy). Data are taken with the HP5372A Frequency and Time Interval analyzer which measures the rf frequency. The instrument measures the frequency every $2.0 \mu \mathrm{s}$ for exactly $10.0 \mathrm{~ms}$. The measured data are subtracted from the target AGS frequency and stored in a fast RAM. The $\Delta f(t)$ data are fetched from the RAM and sent to a direct digital synthesizer using a precise clock at $1.0 \mathrm{MHz}$, (each datum is used twice). Typically for proton operation, $\Delta f(t)$ begins at about $100 \mathrm{kHz}$ and winds down to zero Hertz.

The generator that creates $\Delta \mathrm{f}(\mathrm{t})$ must fulfill some demanding requirements. First, it must be very precise so that the beam frequency, and hence radius, which is now controlled by $\Delta \mathrm{f}(\mathrm{t})$ and the reference from the AGS will be reproducible. Second, it must actually be a vector signal, that is, it must provide two components that are orthogonal. Furthermore the two components must remain orthogonal for all frequencies, even dc. Third, the angle defined by the two components must be the same at dc each time it runs, since this will determine the angle between the Booster beam and the AGS reference signal. Figure 5.4 shows $\Delta \mathrm{f}_{\mathrm{x}}$ and $\Delta \mathrm{f}_{\mathrm{y}}$.

In order for the third requirement to be fulfilled, the $\Delta f(t)$ generator must begin its sweep at the same phase each time and follow precisely the same frequency each time. The final phase is given by

$$
\varphi(t)=\int_{0}^{t} \Delta f(\xi) d \xi+\varphi_{o} .
$$

The total accumulated phase over the $10.000 \mathrm{~ms}$ sweep is approximately 500 $(2 \pi)$. By using a direct digital synthesizer to generate $\Delta f(t)$, the variations in the accumulated phase are less than $\pm 1.3^{\circ}$. This follows from the frequency accuracy of the synthesizer, $\pm 0.75 \mathrm{~Hz}$, and the precision of the clock, $<0.1 \mathrm{ppm}$, that advances the synthesizer through its program.

When a change to the $\Delta f(t)$ program is made (to accommodate a new radial program or a change in the magnetic cycle of the machine, for example) the accumulated phase will also change. In order to keep the same final phase reference, an adjustment to the net phase is made by running the synthesizer for a fixed time $(10 \mu \mathrm{s})$ at a fixed frequency, $\mathrm{f}_{\mathrm{o}}$, to set the initial phase,

$$
\varphi_{o}=f_{o} \times 10 \mu s \text {. }
$$

Once the new $\Delta f(t)$ table is obtained, the phase integral is calculated numerically and the frequency $f_{o}$ is determined. 


$$
\begin{aligned}
& \varphi(t)=\varphi_{0}+\int_{0}^{t} \Delta f\left(t^{\prime}\right) d t^{\prime} \\
& \Delta x(t)=V_{0} \cos (\Delta f(t) t) \\
& \Delta y(t)=V_{0} \sin (\Delta f(t) t) \\
& \left(\begin{array}{l}
x_{M R} \\
y M R
\end{array}\right)=\left(\begin{array}{cc}
\Delta x(t) & \Delta y(t) \\
-\Delta y(t) & \Delta x(t)
\end{array}\right)\left(\begin{array}{l}
X_{\text {Booster }} \\
Y_{\text {Booster }}
\end{array}\right)
\end{aligned}
$$

NO or SLON TRIGEZR

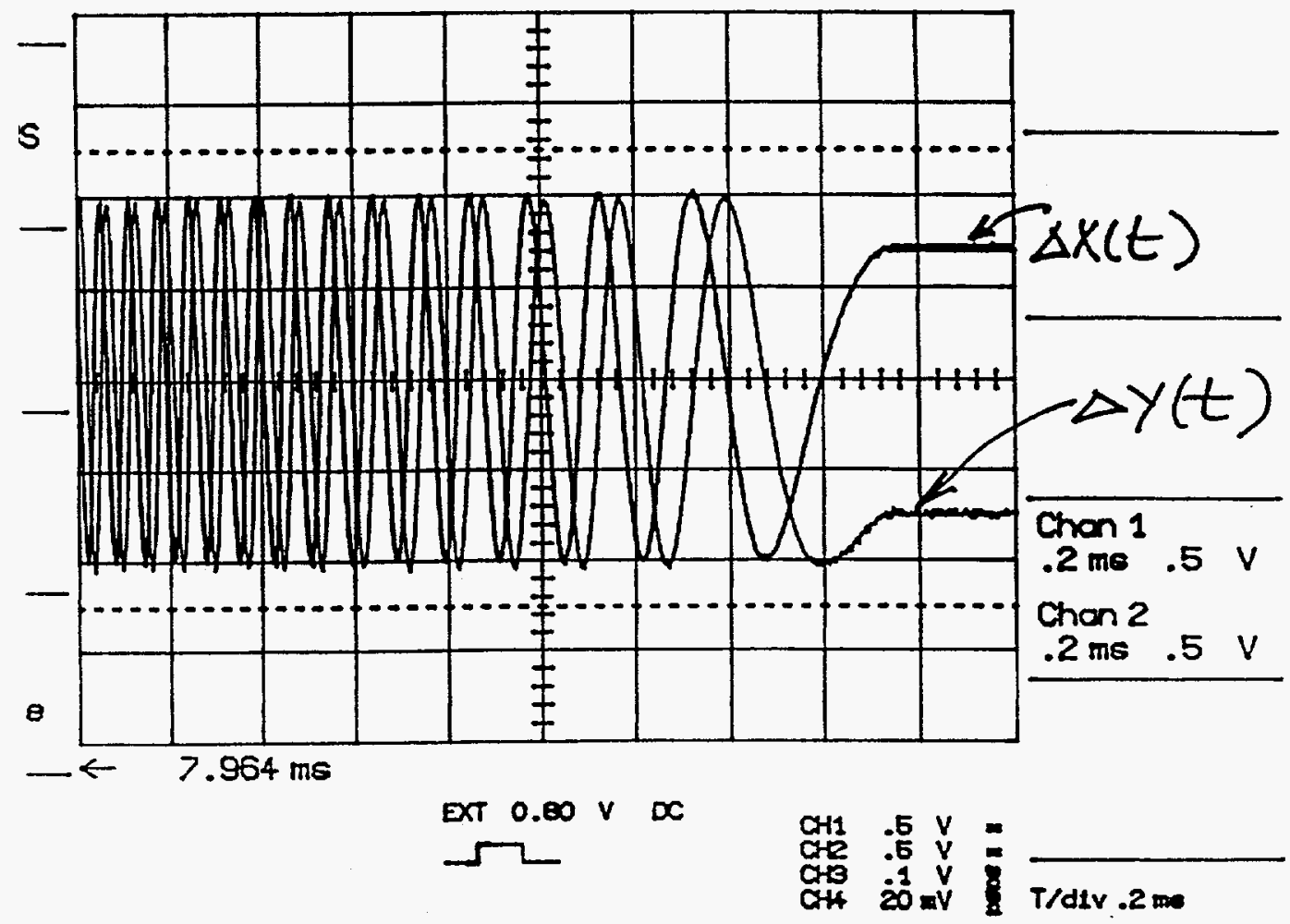

Figure 5.4. The two orthogonal components of the $\Delta f(t)$ signal as the frequency winds down to zero. 
5.3.2 Translation of the Beam Frequency to the Moving Reference Frame. The process of translating the Booster beam frequency to the moving reference frame frequency is essentially a time-dependent rotation of the beam signal vector.

$$
\left[\begin{array}{l}
X_{M R} \\
Y_{M R}
\end{array}\right]=\left(\begin{array}{cc}
\Delta x(t) & \Delta y(t) \\
-\Delta y(t) & \Delta x(t)
\end{array}\right]\left[\begin{array}{c}
X_{\text {Beam }} \\
Y_{\text {Beam }}
\end{array}\right]
$$

The elements of the rotation matrix are the two components of the $\Delta f(t)$ generator. Since the matrix elements are functions of time, the phase angle of the moving reference frame vector with respect to the beam vector increases (decreases) at the frequency of $\Delta \mathrm{f}(\mathrm{t})$. The result is that the moving reference frame frequency is higher (lower) than the beam frequency. When $\Delta f(t)$ reaches zero the angle between the beam and the moving reference is

$$
\varphi\left(t_{f}=10.00 \mathrm{~ms}\right)=\operatorname{Arc} \text { tangent } \frac{\Delta y\left(t_{f}\right)}{\Delta x\left(t_{f}\right)} .
$$

The four products of the matrix multiplication are realized with four-quadrant analog multipliers, AD834.

Figure 5.5 shows the block diagram of the components of the synchronization system. The block labeled "synchro mixer" performs the matrix multiplication.

The beam signal is conditioned before entering the synchro mixer. A lowpass filter converts the bunch signals to a sine wave by removing all Fourier components above the fundamental. A wide dynamic range AGC circuit creates a fixed output level for a $>40 \mathrm{~dB}$ range of beam intensity. A quadruture hybrid transformer provides the orthogonal components of the beam signal.

The reference signal from the AGS is also conditioned. A low-pass filter is used because the signal from the vector sum circuit at the AGS can be distorted in a limiter. The voltage controlled phase shifter sets the phase to which the Booster beam will be locked. An AGC circuit sets a fixed level of the signal sent to the phase detector circuit. 


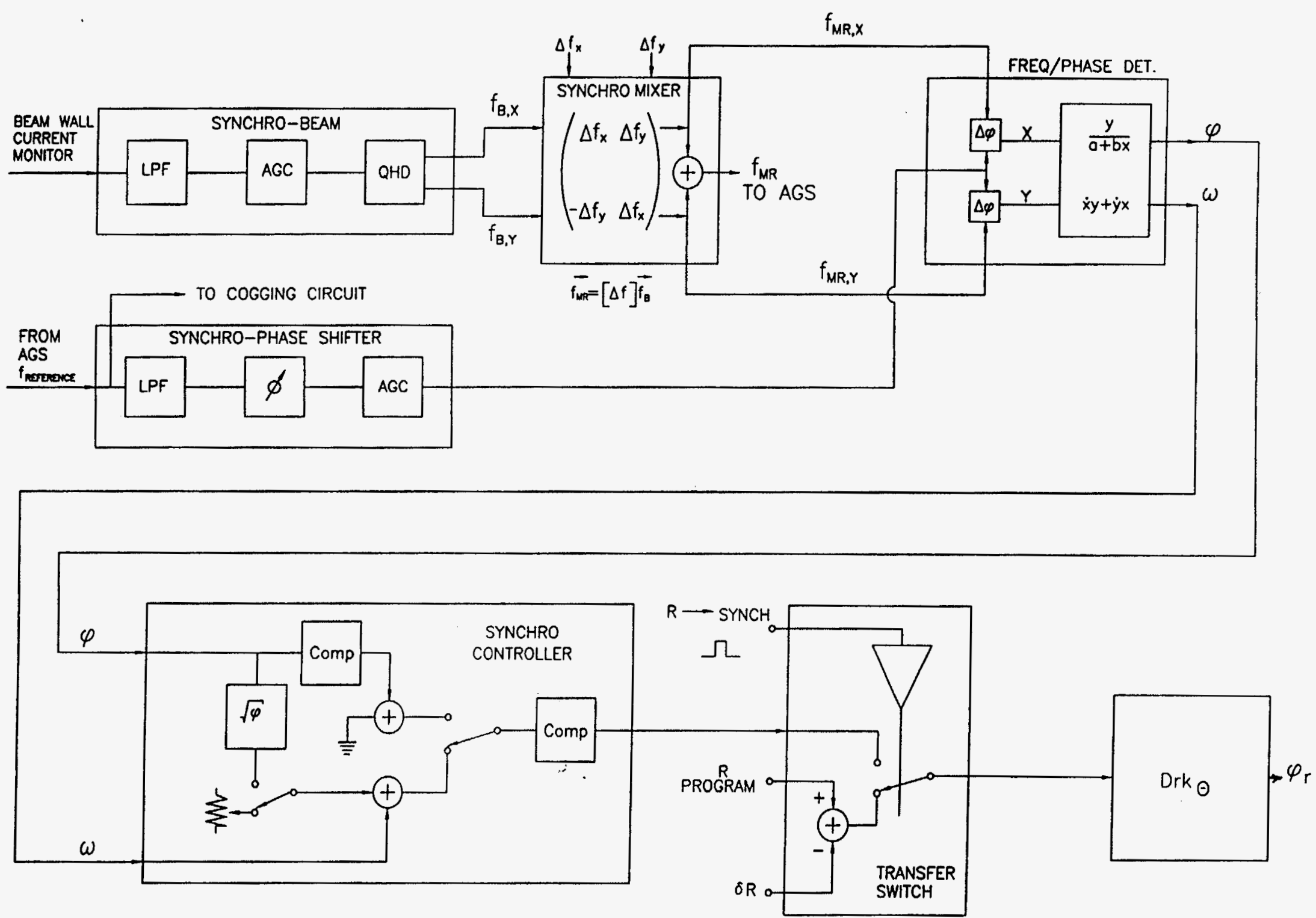

Figure 5.5 "Box" diagram of the moving reference frame synchronization system. Each box is a NIM module. 


\subsection{Frequency/Phase Detector}

The phase is measured in the "Frequency/Phase Detector" module. The moving reference frame components are mixed via double-balanced mixers with the AGS reference signal, giving base-band (dc-coupled) sine and cosine components of the phase angle. The Arc tangent function is approximated by an analog divider realizing the equation

$$
\varphi(t)=\frac{y}{a+b x} .
$$

The parameters a $(=0.5)$ and $b(=0.4)$ are adjusted as a compromise between usable range (approximately $\pm 140^{\circ}$ ) and linearity near zero.

The frequency is calculated from the sine and cosine components by

$$
\omega(t)=x \dot{y}-\dot{x} y,
$$

where: $\quad \omega(t)$ is the difference frequency between the moving reference frame and the AGS reference.

The frequency calculated in this way retains the sign of the frequency, $\omega(t)$. Because $\omega(\mathrm{t})$ is a relative frequency it can meaningfully take on positive or negative values.

The frequency and phase signals go into the "Synchro Controller" which controls the way in which synchronization lock is acquired. 


\subsection{Lock Acquisition in the Synchronization Loop}

Section 4.3 described the dynamics of the synchronization loop. That discussion was limited to the steady-state situation. A transient period must occur, however, when the loop is first closed. The transient will involve the beam and can easily be detrimental, causing emittance blow-up or beam loss. To avoid any detrimental effects on the beam the lock acquisition proceeds in three steps.

Step 1. Radial control is switched off and a frequency loop is closed. In Figure 5.5 the "Transfer Switch" changes state. The regulated frequency in this loop is the frequency of the moving reference frame with respect to the AGS reference signal. That is the frequency measured in the Frequency/Phase Detector circuit described above. The reference value of the frequency loop is a dc level, set via a front panel pot on the Synchro Controller. A value for the reference is chosen that creates a convenient beat frequency, on the order of $-1 \mathrm{kHz}$.

The purpose of establishing a controlled beat frequency is two-fold. One, since the output of the phase detector is a sawtooth wave, the sign of the beat frequency determines whether the slope of the sawtooth is high or low when the wave crosses zero with the correct sign for negative feedback (the gain of the frequency loop is proportional to this slope). Second, the magnitude of the beat frequency determines the time interval between zero crossing, that is, the time one would have to wait before going to the next stage of acquisition. Although a high beat frequency would mean a short time to wait it would also mean a large change in the synchrotron's synchronous phase angle when the synchro loop is finally closed. A beat frequency of $-1 \mathrm{kHz}$ causes a change in the synchronous phase of approximately three degrees.

Step 2. The dc reference for the frequency loop is replaced with a signal proportional to the square root of the phase of the moving reference frame signal with respect the AGS reference signal. See Figure 5.5. The switch in the "Synchro Controller" between the dc reference (pot) and the square root of phase circuit changes state. This is the phase measured in the Frequency/Phase Detector circuit. The purpose of this step is to control the way the phase and frequency approach zero. By applying the square root of the phase to the reference of the frequency loop the loop will synthesize the equation

$$
\omega(t)=\frac{d \varphi(t)}{d t}=k \sqrt{\varphi(t)} .
$$


If the coefficient $\mathrm{k}$ is fixed to be $\omega(0) / \sqrt{\varphi(o)}$, where $\omega(0)$ and $\varphi(0)$ are the frequency and phase when the loop is closed, then the equation has the solution

$$
\varphi(t)=\frac{1}{4} \frac{\omega_{(o)}^{2}}{\varphi(o)} t^{2}+\omega(o) t+\varphi(o),
$$

and the frequency is given by

$$
\omega(t)=\frac{1}{2} \frac{\omega_{(o)}^{2}}{\varphi(0)} t+\omega(0) .
$$

Since $\omega(0)$ was set to a negative value by the frequency loop the phase will decrease quadratically to zero while the frequency increases linearly toward zero. At $t=-2 \varphi(0) / \omega(0)$, the phase and frequency reach zero simultaneously and the synchronization loop can be closed with no first order or second order transient. In third order there is a transient, $\mathrm{d}^{2} \varphi / \mathrm{dt}^{2} \neq 0$. This is the origin of the approximately three degree change in the synchronous phase angle mentioned above. Figure 5.6 illustrates the behavior of the frequency and phase during steps one and two.

Step 3. The synchronization loop is closed. The other switch in the Synchro Controller changes state. Note that the reference value for this loop is zero. The actual phase between the bunches and the buckets is controlled via the phase shifter in the Synchro-Phase Shifter module. See Figure 5.1. This assures that the same point on the transfer curve of the phase detector's approximation to the Arc tangent will always be used, namely, $y=\sin (\varphi)=0$ and $x=\cos (\varphi)=1$.

Once the synchronization loop is closed the phase of the bunches will be controlled by the $\Delta \mathrm{f}(\mathrm{t})$ synthesizer. Extraction could, in principle, take place at any time after the synchronization loop is closed. As long as the time of extraction repeats precisely with respect to the time the $\Delta f(t)$ synthesizer starts running, then the phase of the beam will be reproducible.

Figure 5.7 shows the output of the phase detector during operation with beam. Also shown in the figure are the beam radius signal and the triggers that advance the process from steps one to two and two to three.

Figure 5.8 shows the details of the Synchro Controller. The switches can be seen and also the logic circuits that control the states of the switches are illustrated. 


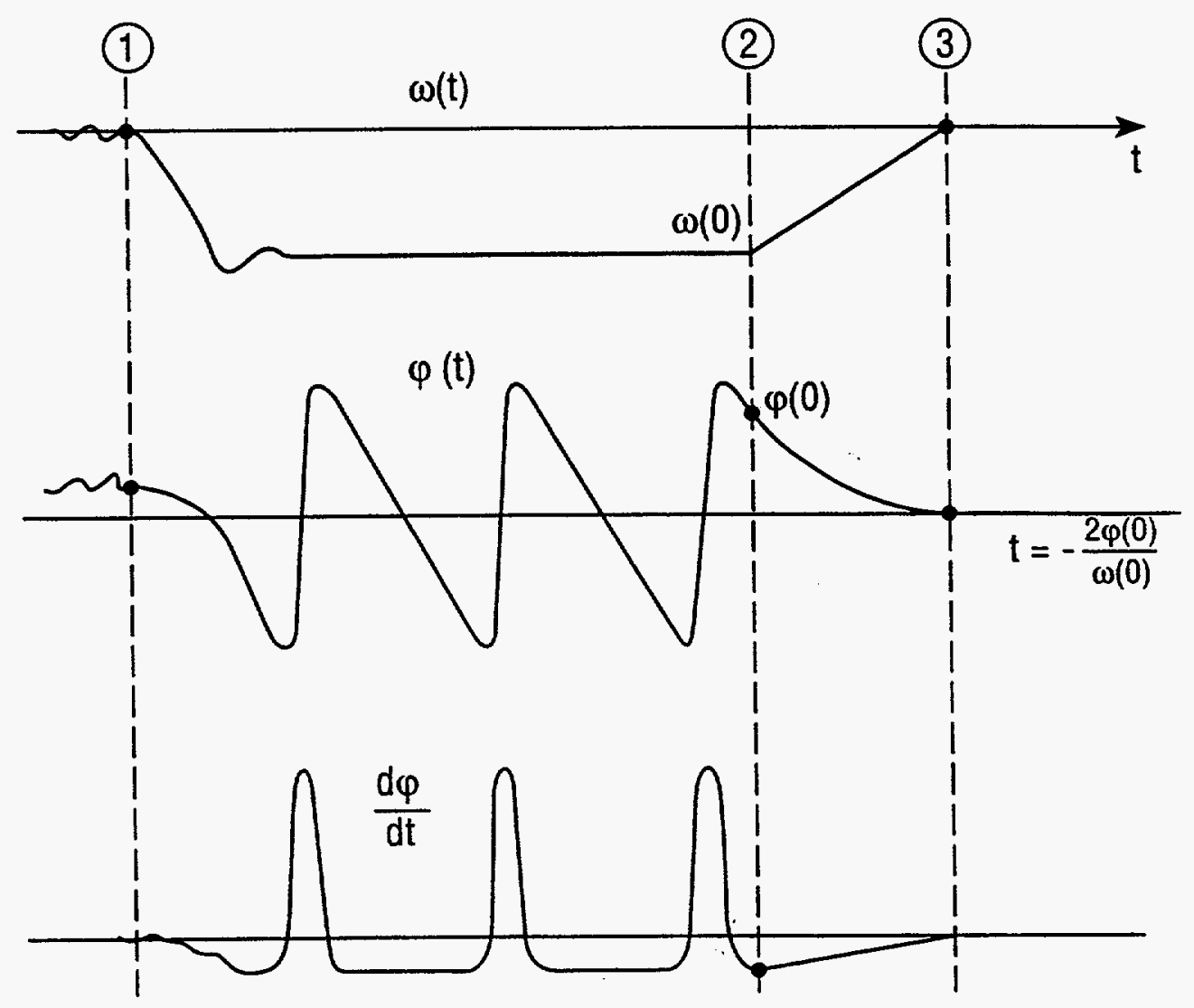

Figure 5.6. An illustration of the frequency, phase, and phase derivative signals during the lock acquisition sequence. The derivative of the phase detector signal (which is not equal to frequency because of the limited range of the phase detector) is used to trigger the switch to square root of phase for the reference of the frequency loop, at point 2. 


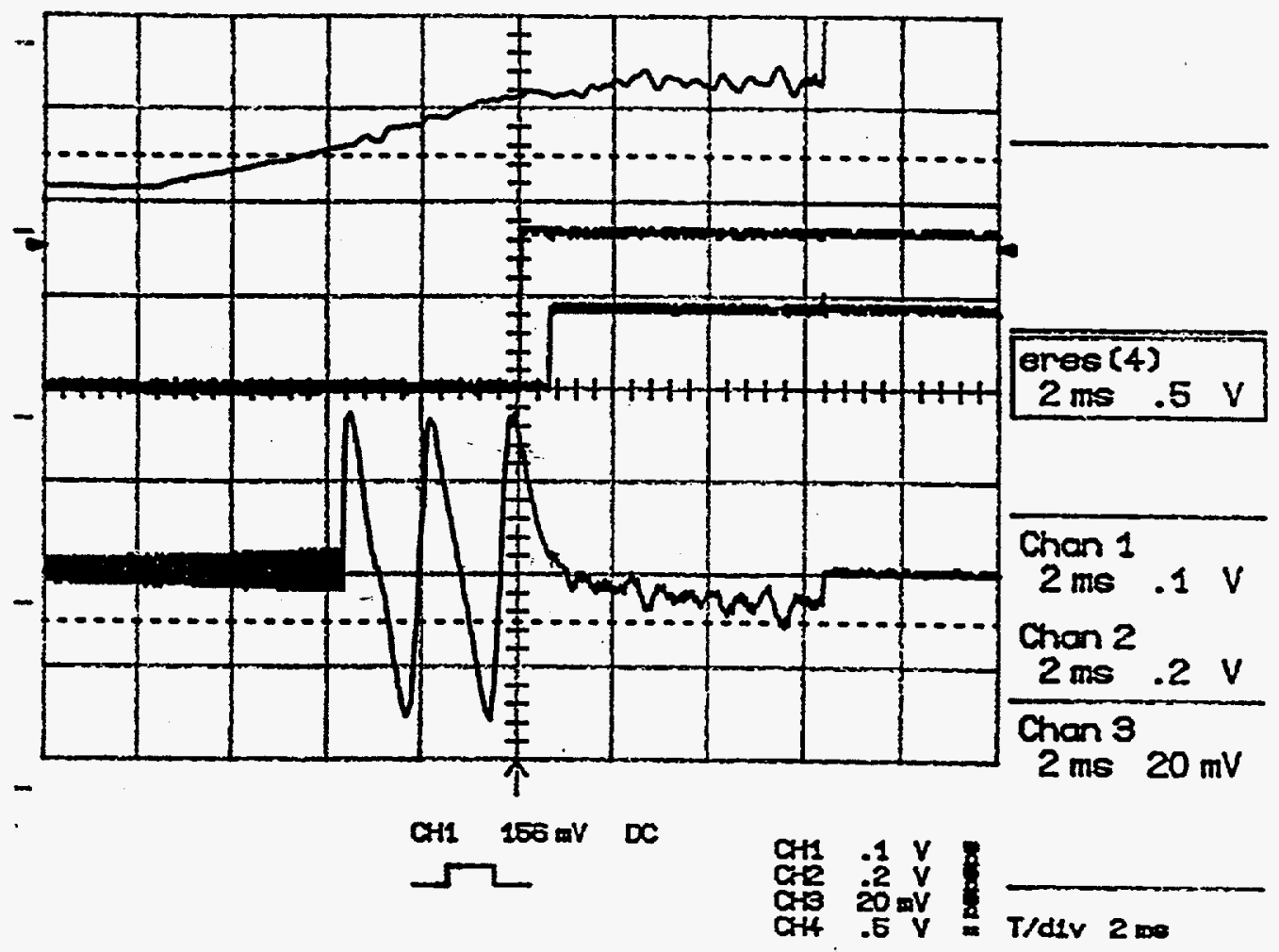

Figure 5.7. The phase signal when the synchronization system is in operation with proton beam, bottom. Other traces are beam radius, top, and triggers that advance the system from steps 1 to 2 and 2 to 3. 


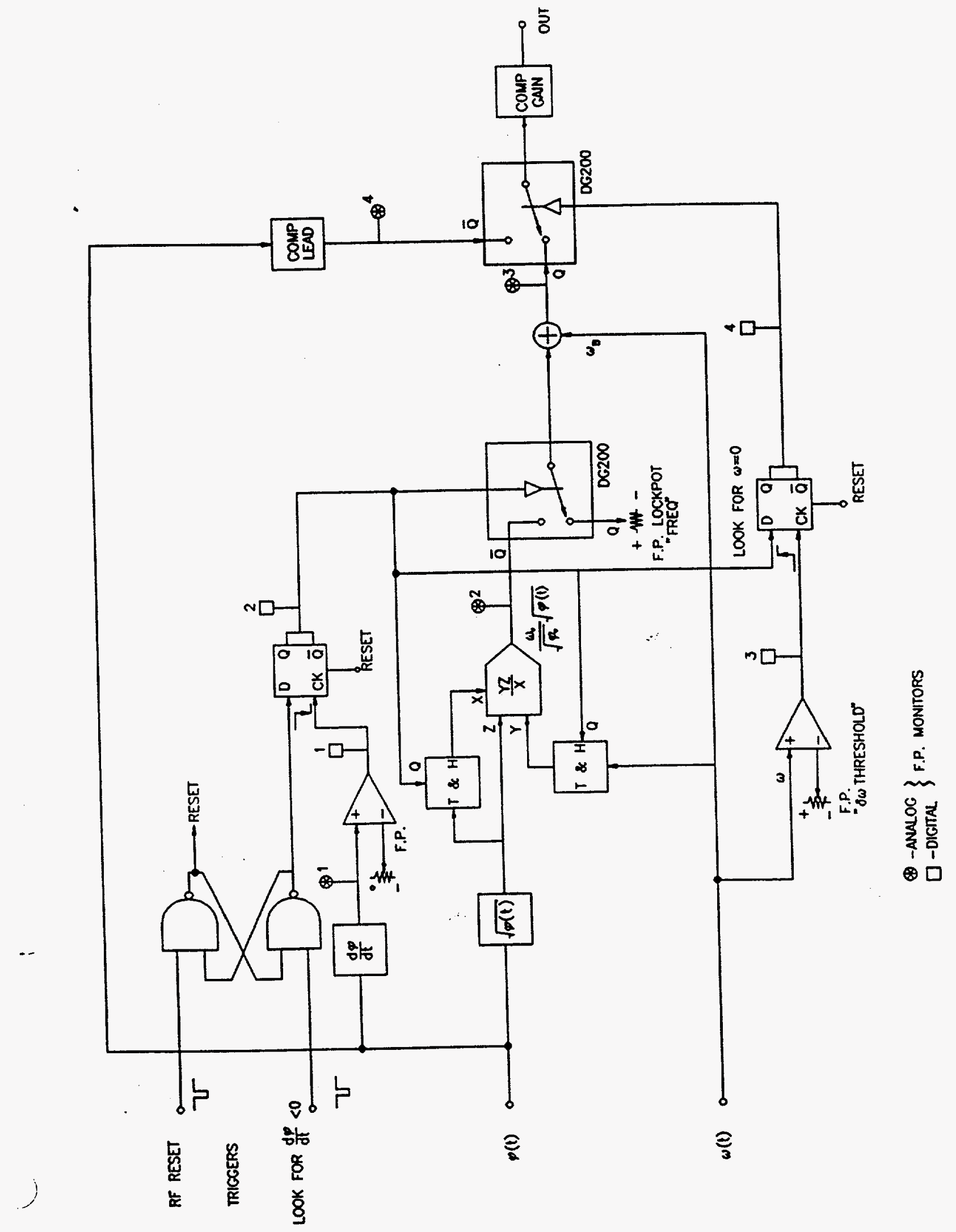

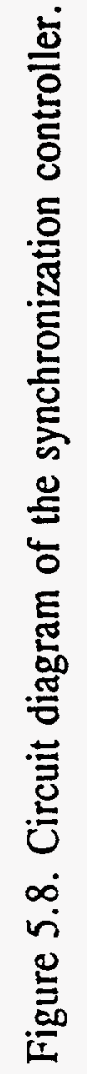


The decision to switch to state two is based on determining when the phase detector output is on the proper side of the saw tooth wave form. This is done by calculating the derivative of the phase signal and detecting a negative value. This derivative of phase is not actually frequency because of the approximation to the Arc tangent. In Figure 5.6 the derivative of the phase signal is also plotted. The point where the switch to step two occurs is indicated. An input trigger sets the latch and the next time the derivative signal crosses the threshold in the proper direction the switch state is changed. The input trigger is arranged to occur after approximately two cycles of the beat frequency.

Once the first switch has changed state the second switch, to step three, is ready to change as soon as the frequency reaches zero.

The proper value of the constant $\mathrm{k}$ is obtained from the running signals $\omega(\mathrm{t})$ and $\sqrt{\varphi(t)}$ by track and hold (T \& $\mathrm{H}$ in the figure) circuits. The signal that then goes to the reference of the frequency loop is then scaled by the ratio of these constants in an YZ/X analog multiplier. This insures that the proper scaling constant will be used to make the phase signal continuous at the switch to step two even if other adjustments are made. For example, adjustments could be made to the beat frequency or threshold of the phase derivative detector. 


\section{CONCLUSIONS}

The beam control system for the AGS Booster has been described in detail and analyzed from the viewpoint of a servo system with the beam radius as a function of time being the reference input to the servo. In order to achieve stability and good response characteristics, the inherent synchrotron oscillations of the beam must be damped by feedback before the radius servo can operate. This feedback is called the phase loop and is the most important loop of the system. The performance of the phase loop is limited by time delays that originate from three causes: cable delays to and from the rf cavities, response time of the rf cavities, and time averaging of pick-up signals which measure the beam phase.

To minimize tracking errors in the servo, considerable use is made of feedforward techniques. A very good approximation to the rf frequency is made by a system called the frequency program. The frequency program comprises a table of revolution frequency data, as a function of average magnetic field in the ring magnets, and a direct digital synthesizer which produces the if frequency for any harmonic number plus $10.7000 \mathrm{MHz}$. The actual rf frequency is generated in the heterodyne system that subtracts a nominal $10.7 \mathrm{MHz}$ signal from the synthesizer's output. The exact value of the $10.7 \mathrm{MHz}$ frequency is controlled by the phase loop and the radius servo.

Other feedforward systems provide approximations to such quantities as the synchronous phase angie as a function of acceleration rate and rf voltage, $\mathrm{rf}$ drive power level for the rf cavities, ferrite bias tuning current to keep the rf cavities in resonance.

When the Booster must be synchronized to the AGS for bunch-to-bucket transfer, the servo on the radius is replaced with a servo of Booster beam phase with respect to AGS if voltage. The dynamics of the phase servo have been analyzed with special consideration given to the techniques that allow the switch over from radius to phase servo without destructive transients being introduced in the beam dynamics.

Extraction from the Booster typically takes place at the full acceleration rate, while the beam frequency is changing rapidly. This places special demands on the synchronization system since the phase must be controlled between two unequal and changing frequencies. The system that solves this problem is called the moving reference frame system and is described in detail. It employs a direct digital synthesizer to create a precise phase-resolved beat frequency between the Booster and AGS rf systems.

The Booster if beam control system has worked successfully for many months of production-running operation and has achieved the goal of requiring very little operator adjustments. To a great extent, the only operator interaction with the rf beam control system is to modify the radius as a function of time as desired. 


\section{REFERENCES}

1. D. Boussard, Une Presentation Elementaire du Systeme "Beam Control" du PS, MPS/SR/Note 73-10.

2. W. Schnell, Equivalent Circuit Analysis of Phase Lock Beam Control System, CERN 68-27 ISR Division, July 1968.

3. U. Bigliani, The Beam Control System for the CERN PS Booster, IEEE Trans. Nucl. Sci. NS-18(3), 352 (1971).

4. D. Boussard, Design of a Ring RF System; CERN School, Oxford, England, 1991, CERN 92-03 11, June 1992, Vol. II.

5. E. Raka, RF System Considerations for a Large Hadron Collider AIP Conference Proceedings 184, 1989, Vol. 1, pg. 289.

6. S. Koscielniak, RF System Aspects of Longitudinal Beam Control (in the Low Current Regime), AIP Conference Proceedings 249, 1992, Vol. 1, pg. 89.

7. S.P. Jachim, R.C. Weber, R.E. Shafer, An RF Beam Position Measurement Module for the Fermilab Energy Doubler, IEEE Trans. Nucl. Sci. Vol. NS-28, No: 3, June 1981.

8. E. Beadle, et al., Design of the AGS Booster Beam Position Monitor System, IEEE Particle Accelerator Conference, Chicago Il, 1989, pg. 1536.

9. L. Ahrens, AGS Wall Current Monitor, private communication.

10. R. Weber, Measurement Techniques Including Design of a Wall Current Monitor, AIP Conference Proceedings 212, Accelerator Instrumentation, Upton, New York, 1989, pg. 85.

11. J. Geller, Booster Gauss Clock, private communication.

12. J.E. Griffin, The Effect of Large Delays on Beam RF Phase Lock Loops, IEEE Trans. Nucl. Sci. NS-26, No. 3, June 1979.

13. Control System Development Using Dynamic Signal Analyzers, HewlettPackard Application Note 243-2, 1984, pg. 38. 


\section{APPENDIX}

\subsection{Final Value Theorem for the Radial Loop}

In Section 4.2.3, we calculated the residual error in the radial loop using the final value theorem by evaluating the transfer function for the radial error at zero frequency. Here we show why that is correct for the special case when the change in the reference to the radial servo is a step function.

If $F(s)$ is the Laplace transform of $f(t)$ and $s F(s)$ has poles only in the left half s-plane, then $f(\infty)$ is given by

$$
\operatorname{limit}_{t \rightarrow \infty} f(t)=\operatorname{limit}_{s \rightarrow 0} s F(s)
$$

which is the final value theorem that follows directly from the definition of the Laplace transform and the property

$$
s F(s)-f\left(0^{-}\right)=\int_{0^{-}}^{\infty} e^{-s t} \frac{d f}{d t} d t .
$$

In our case $F(s)$ is the transfer function of the relative radial position error, derived in Section 4.2. For the case of a step function input,

$$
\mathrm{R}(\mathrm{t})=\mathrm{R}_{\mathrm{o}} u(\mathrm{t})
$$

where $u(\mathrm{t})$ is the unit step at $\mathrm{t}=0$, the relative residual error,

$$
\operatorname{limit}_{t \rightarrow \infty}\left[\frac{R(t)-\delta R(t)}{R_{o}}\right],
$$

is given by

$$
\operatorname{limit}_{s \rightarrow 0} s\left(\frac{R(s)-\delta R(s)}{R_{o}}\right) .
$$


Using $R(s)=R_{0} / s$ and

$$
\delta R(s)=\frac{G r(s)}{1+G r(s)} R(s),
$$

we have

$$
\operatorname{limit}_{t \rightarrow \infty}\left[\frac{R(t)-\delta R(t)}{R_{o}}\right]=\operatorname{limit}_{s \rightarrow o} \frac{s}{R_{o}}\left[\frac{R_{o}}{s}-\frac{G r(s)}{1+G r(s)} \frac{R_{o}}{s}\right]=\operatorname{limit}_{s \rightarrow o} \frac{1}{1+G r(s)}
$$




\subsection{Feedback Loop Calculations with Program CC}

The numerical calculations for the control system design for this report were performed with a commercial code named Program CC. It was produced by the Systems Technology, Inc. Hawthorne, California. The program operates either in the state-variable representation or the classical transfer function representation. All calculations done here used the transfer function representation to facilitate correspondence with laboratory measurements.

8.2.1 Sensitivity Constants. Table 8.1 lists the sensitivity constants of the system used in the calculations. The names of the constants, corresponding to symbols used in Figure 4.22, are given, together with the variable names used in the program calculations. The description explains its function. Numerical values are shown.

Table 8.1

\begin{tabular}{|c|c|c|c|}
\hline Constant & $\begin{array}{l}\text { Program } \\
\text { Name }\end{array}$ & Description & Numerical Value \\
\hline $\mathrm{k}_{\varphi}$ & KPHI & $\begin{array}{l}\text { Bunch-to-bucket } \\
\text { phase detector }\end{array}$ & 0.53 Volts/radian \\
\hline $\mathrm{k}_{\mathrm{o}}$ & Ko & VCO constant & $2.3 \times 10^{5} \mathrm{~s}^{-1} /$ Volt \\
\hline $\mathrm{k}_{\mathrm{pu}}$ & KPU & Radius pick-up & $0.05 \mathrm{Volt} / \mathrm{mm}$ \\
\hline $\mathrm{k}_{\theta}$ & KSHIFT & Phase shifter & $\begin{array}{l}0.87 \mathrm{radian} / \text { Volt } \\
\left(50^{\circ} / \text { Volt }\right)\end{array}$ \\
\hline $\mathbf{k}_{\omega}$ & KOMEGA & $\begin{array}{l}\text { Frequency } \\
\text { discriminator }\end{array}$ & $\begin{array}{l}5.3 \times 10^{-4} \mathrm{Volt} / \mathrm{s}^{-1} \\
(10 \mathrm{Volts} / 3 \mathrm{kHz})\end{array}$ \\
\hline $\mathrm{k}_{\mathrm{s}}$ & KSYNC & $\begin{array}{l}\text { Synchro phase } \\
\text { detector }\end{array}$ & $\begin{array}{l}1.0 \text { Volt } / \text { radian } \\
\left(3.1 \text { Volts } / 180^{\circ}\right)\end{array}$ \\
\hline b & --- & $\begin{array}{l}\text { Scale factor of } \\
\text { radius beam transfer } \\
\text { function }\end{array}$ & $\begin{array}{l}3 \times 10^{5} \mathrm{~mm} / \mathrm{s}^{-1} \\
\text { to } \\
3 \times 10^{4} \mathrm{~mm} / \mathrm{s}^{-1}\end{array}$ \\
\hline
\end{tabular}


8.2.2. Program CC Transfer Function Names. Program CC operates on objects called transfer functions, which are complex-valued functions of the complex Laplace transform variable $s$. There are no special objects to represent constants; therefore, the constants of the system, such as the sensitivity constants listed in Table 8.1 and the synchrotron frequency, are treated as transfer functions of order zero in $\mathrm{s}$.

Transfer functions that describe the components of the beam control system are listed here. Refer to Table 8.1 for the sensitivity constants. Transfer functions describing subsystems, such as the phase or radial loops, are created by combining transfer functions of components. Subsystems are described below in Section 8.2.3

\section{1. $\mathrm{e}^{-\mathrm{s} \tau}$, ESTAU}

Time delay, given by Padé approximation of order $\mathrm{n}$ to the complex exponential for delay $\tau, \operatorname{PADE}(\mathrm{n}, \tau)$.

2. $\mathrm{C}(\mathrm{s}), \mathrm{COFS}$

Delayed frequency modulation response of the cavities.

$$
\mathrm{COFS}=\mathrm{ESTAU} /\left(\mathrm{s} /\left(2 \pi \times 20 \times 10^{3}\right)+1\right)
$$

3. $\mathrm{D} \varphi(\mathrm{s}), \mathrm{DPHI}$

The PID phase loop compensator. Its input comes from the bunch-tobucket phase detector. Its output goes to the VCO. See Figure 4.6 and Equation 4.5

$$
D P H I=\frac{86(2.5 e-5 s+1)(3.7 e-5 s+1)(-7.7 e-7 s+1)}{(0.01 s+1)(3.3 e-6 s+1)}
$$

4. $\omega_{s}$, WS1 and WS2

Maximum and minimum values of the synchrotron frequency.

$$
\begin{aligned}
& \mathrm{WS} 1=2 \pi \times 7 \mathrm{e} 3, \text { injection } \\
& \mathrm{WS} 2=2 \pi \times 1 \mathrm{e} 3, \text { extraction }
\end{aligned}
$$


5. $\mathrm{B}_{\varphi}(\mathrm{s}), \mathrm{B} 1 \mathrm{PHI}$ and $\mathrm{B} 2 \mathrm{PHI}$

Beam transfer function of rf frequency modulations to bunch-to-bucket phase, for maximum and minimum synchrotron frequencies, see Equation 1.3 .

$$
\begin{aligned}
& \mathrm{B} 1 \mathrm{PHI}=\mathrm{s} /\left(\mathrm{s}^{2}+\mathrm{WS}^{2}\right) \\
& \mathrm{B} 2 \mathrm{PHI}=\mathrm{s} /\left(\mathrm{s}^{2}+\mathrm{WS}^{2}\right)
\end{aligned}
$$

6. $B_{\omega}(s), B 1 W$ and $B 2 W$

Beam transfer function of rf frequency modulation to beam frequency, see Equation 1.5.

$$
\begin{aligned}
& \mathrm{B} 1 \mathrm{~W}=\mathrm{WS} 1^{2} /\left(\mathrm{s}^{2}+\mathrm{WS} 1^{2}\right) \\
& \mathrm{B} 2 \mathrm{~W}=\mathrm{WS} 2^{2} /\left(\mathrm{s}^{2}+\mathrm{WS} 2^{2}\right)
\end{aligned}
$$

7. $B_{R}(s), B 1 R$ and $B 2 R$

Beam transfer function of $\mathrm{rf}$ frequency modulations to beam radius, see Equation 1.4. The constant $b$ in Equation 1.4 is evaluated for injection and extraction energies, and used with the maximum and minimum synchrotron frequencies, respectively.

$$
\begin{aligned}
& \mathrm{B} 1 \mathrm{R}=-3 \mathrm{e} 5 /\left(\mathrm{s}^{2}+\mathrm{WS} 1^{2}\right) \\
& \mathrm{B} 2 \mathrm{R}=-3 \mathrm{e} 4 /\left(\mathrm{s}^{2}+\mathrm{WS} 2^{2}\right)
\end{aligned}
$$

8. $\mathrm{D}_{\mathrm{r}}(\mathrm{s})$, DROFS

The radial loop compensator, see Figure 4.15.

$$
\text { DROFS }=8 /(s /(2 \pi \times 1 e 3)+1)
$$

9. $\mathrm{D}_{\omega}(\mathrm{s})$, DOMEGA

The frequency loop compensator. It works in cascade with the radial loop compensator and is just a constant, see Section 4.3.1.

$$
\text { DOMEGA }=0.005
$$


10.

$\mathrm{D}_{\mathrm{s}}(\mathrm{s})$, DSYNC

The synchronization loop compensator, see Figure 4.25 .

$$
D S Y N C=\frac{0.08(5 e-4 s+1)}{(9 e-6 s+1)(2.4 e-6 s+1)}
$$

8.2.3 Program CC Transfer Function for Subsystems. The transfer functions of the previous sections are combined to build transfer functions for subsystems. Open loop subsystems, such as $\mathrm{G}(\mathrm{s})$, are comprised of strings of products. Closed loop subsystems use a special syntax of the program called the feedback operator, 1. It is a shorthand for the algebra

$$
(\alpha \mid \beta)=\frac{\alpha}{1+\beta \alpha}
$$

where $\alpha$ and $\beta$ are functions of $s$.

1. Phase Loop

a. $\quad G(s)=k_{\varphi} k_{o} C(s) D_{\varphi}(s) B_{\varphi}(s) e^{-\tau_{d} s}$

Eq. 4.2

GP1OFS $=\mathrm{KPHI} * \mathrm{~K} 0 *$ COFS $* \mathrm{DPHI} * \mathrm{~B} 1 \mathrm{PHI}$

GP2OFS $=\mathrm{KPHI} * \mathrm{~K} 0 * \mathrm{COFS} * \mathrm{DPHI} * \mathrm{~B} 2 \mathrm{PHI}$

b. $\quad\left[\frac{\omega_{r f}}{\varphi_{r}}\right]=\frac{k_{\varphi} k_{o} C(s) D_{\varphi}(s) e^{\tau_{d} s}}{1+G(s)}$

Section 4.1.2

PRTOW1 $=(\mathrm{KPHI} * \mathrm{~K} 0 * \mathrm{COFS} * \mathrm{DPHI}) \mid \mathrm{B} 1 \mathrm{PHI}$

PRTOW2 $=(\mathrm{KPHI} * \mathrm{~K} 0 * \mathrm{COFS} * \mathrm{DPHI}) \mid \mathrm{B} 2 \mathrm{PHI}$

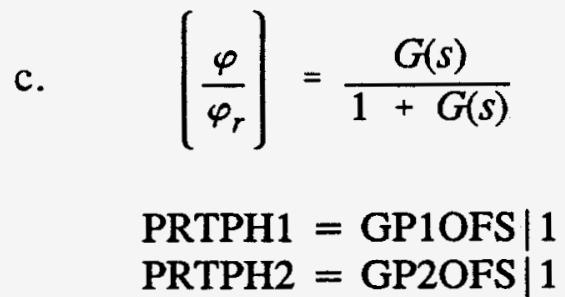

Section 4.1.1 
2. Radial Loop

a. $\quad G r(s)=k_{\theta} k_{p u} \operatorname{Dr}(s)\left[\frac{\delta \omega_{r f}}{\varphi_{r}}\right] B_{R}(s)$

Eq. 4.11

$$
\begin{aligned}
& \text { GR1OFS }=\text { KSHIFT } * \text { KPU } * \text { DROFS } * \text { PRTOW } 1 * B 1 R \\
& \text { GR2OFS }=\text { KSHIFT } * \text { KPU } * \text { DROFS } * \text { PRTOW } 2 * B 1 R
\end{aligned}
$$

b Closed loop radius servo

$$
\begin{aligned}
& \mathrm{VR} 1=\mathrm{GR} 1 \mathrm{OFS} \mid 1 \\
& \mathrm{VR} 2=\mathrm{GR} 2 \mathrm{OFS} \mid 1
\end{aligned}
$$

3. Frequency Loop

a. Open loop

$$
G \omega(s)=k_{\omega} k_{\theta} D_{\omega} \operatorname{Dr}(s) B_{\omega}(s)\left(\frac{\delta \omega_{r f}}{\varphi_{r}}\right)
$$

GF1OFS $=$ KOMEGA $*$ KSHIFT $*$ DOMEGA $*$ DROFS*B1W*PRTOW1 GF2OFS $=$ KOMEGA $*$ KSHIFT $*$ DOMEGA $*$ DROFS $*$ B2W $*$ PRTOW 2

b. Closed loop

VOMEG1 $=$ GF1OFS $\mid 1$

VOMEG2 = GF2OFS $\mid 1$ 
4. Synchronization Loop

a. Open loop

$$
G_{s}(s)=\left(\frac{k_{s}}{s}\right) k_{\theta} D_{s}(s) D_{\omega} D_{r}(s) B_{\omega}(s)\left(\frac{\delta \omega_{r f}}{\varphi_{r}}\right)
$$

GS1OFS $=$ KSYNC $*(1 / s) *$ KSHIFT*DSYNC*DOMEGA*DROFS*B1W*PRTOW 1

GS2OFS $=$ KSYNC* $(1 / \mathrm{s}) * \mathrm{KSHIFT} * \mathrm{DSYNC} *$ DOMEGA*DROFS*B2W*PRTOW2

b. Closed loop

$$
\begin{aligned}
& \text { VSYNC1 }=\text { GS1OFS } \mid 1 \\
& \text { VSYNC2 }=\text { GS2OFS } \mid 1
\end{aligned}
$$

8.2.4 Calculation of Phase Response to Radial Step. In Section 4.2.2, we show the bunch-to-bucket phase response to a step change in the reference function of closed radial loop. This response is a transient that settles to zero when the beam stabilizes at the new radius. The magnitude and duration of this transient are illustrated in Figure 4.2.1

The formalism of the calculation is described here. First, write the response of the phase correction, $\varphi_{\mathrm{r}}$,

$$
\varphi_{r}=k_{\theta} D_{r}(s)(R(t)-\delta R) .
$$

But,

$$
\delta R=\frac{G_{r}(s)}{1+G_{r}(s)} R(s)
$$


So, $\varphi_{\mathrm{r}}$ as a function $\mathrm{R}(\mathrm{s})$ is

$$
\varphi_{r}=k_{\theta} D_{r}(s)\left[1-\frac{G_{r}}{1+G_{r}}\right] R(s),
$$

and we have

$$
\varphi=\frac{G(s)}{1+G(s)} \varphi_{r}
$$

so that

$$
\varphi=\frac{G(s)}{1+G(s)} \frac{k_{\theta} D_{r}(s)}{1+G_{r}(s)} R(s) .
$$

In terms of the Program CC transfer functions, this is easily written:

$$
\begin{aligned}
& \varphi_{\mathrm{r}}=\mathrm{KSHIFT} * \mathrm{DROFS} *(1-\mathrm{VR} 1) * \mathrm{R} \\
& \varphi=\mathrm{PRTPH} 1 * \varphi_{\mathrm{r}} \\
& \text { P1BEAM }=\text { PRTPH } 1 * \text { KSHIFT } * \text { DROFS } *(1-\mathrm{VR} 1)\left(\mathrm{R}_{\mathrm{o}} / \mathrm{s}\right) \\
& \text { P2BEAM }=\text { PRTPH } 2 * \text { KSHIFT } * \text { DROFS }(1-\mathrm{VR} 2) *\left(\mathrm{R}_{\mathrm{o}} / \mathrm{s}\right)
\end{aligned}
$$

